CERN-TH/97-15

ROME prep. 1163/97

ROM2F $/ 97 / 23$

\title{
Improved Renormalization of Lattice Operators: A Critical Reappraisal
}

\author{
M. Crisafulli ${ }^{a}$, V. Lubicz ${ }^{b}$ and A. Vladikas ${ }^{c, d}$ \\ ${ }^{a}$ Dip. di Fisica, Univ. di Roma "La Sapienza" and INFN, Sezione di Roma, \\ P.le A. Moro 2, I-00185 Roma, Italy. \\ ${ }^{b}$ Dip. di Fisica, Univ. di Roma Tre and INFN, Sezione di Roma, \\ Via della Vasca Navale 84, I-00146 Roma, Italy \\ ${ }^{c}$ Theory Division, CERN, 1211 Geneva 23, Switzerland. \\ ${ }^{d}$ INFN, Sezione di Roma II, and Dip. di Fisica, Univ. di Roma "Tor Vergata", \\ Via della Ricerca Scientifica 1, I-00133 Roma, Italy.
}

\begin{abstract}
We systematically examine various proposals which aim at increasing the accuracy in the determination of the renormalization of two-fermion lattice operators. We concentrate on three finite quantities which are particularly suitable for our study: the renormalization constants of the vector and axial currents and the ratio of the renormalization constants of the scalar and pseudoscalar densities. We calculate these quantities in boosted perturbation theory, with several running boosted couplings, at the "optimal" scale $q^{*}$. We find that the results of boosted perturbation theory are usually (but not always) in better agreement with non-perturbative determinations of the renormalization constants than those obtained with standard perturbation theory. The finite renormalization constants of two-fermion lattice operators are also obtained non-perturbatively, using Ward Identities, both with the Wilson and the tree-level Clover improved actions, at fixed cutoff ( $\beta=6.4$ and 6.0 respectively). In order to amplify finite cutoff effects, the quark masses (in lattice units) are varied in a large interval $0 \lesssim a m \lesssim 1$. We find that discretization effects are always large with the Wilson action, despite our relatively small value of the lattice spacing $\left(a^{-1} \simeq 3.7 \mathrm{GeV}\right)$. With the Clover action discretization errors are significantly reduced at small quark mass, even though our lattice spacing is larger $\left(a^{-1} \simeq 2 \mathrm{GeV}\right)$. However, these errors remain substantial in the heavy quark region. We have implemented a proposal for reducing $\mathcal{O}(a m)$ effects, which consists in matching the lattice quantities to their continuum counterparts in the free theory. We find that this approach still leaves appreciable, mass dependent, discretization effects.
\end{abstract}

CERN-TH/97-15

July 1997 


\section{Introduction}

One of the major goals of lattice QCD computations is the non-perturbative evaluation of hadronic Matrix Elements (ME's) which are relevant for strong and electroweak interactions. On the lattice, the ME's of bare operators are computed at finite UV cutoff $a^{-1}$ ( $a$ is the lattice spacing) by simulating QCD at fixed bare coupling $\beta=6 / g_{0}^{2}$. These ME's are functions of the finite cutoff. In order to extract physical information from them, the lattice operators require renormalization. For example, the $\rho$-meson decay constant $f_{\rho}^{-1}$ is obtained from the $\mathrm{ME}\left\langle 0\left|V_{k}(0)\right| \rho_{r}\right\rangle$ as follows:

$$
\epsilon_{k}^{r} \frac{m_{\rho}^{2}}{f_{\rho}}=Z_{V}\left\langle 0\left|V_{k}(0)\right| \rho_{r}\right\rangle
$$

where $m_{\rho}$ is the meson mass and $\epsilon_{k}^{r}$ is its polarization vector. $V_{k}$ is a spatial component of the bare (lattice) vector current and $Z_{V}$ is its renormalization constant (RC). $Z_{V}$ connects the lattice theory at finite cutoff to the renormalized continuum theory. Analogously, the $\pi$-meson's decay constant $f_{\pi}$ is determined from the ME of the axial current through:

$$
i f_{\pi} m_{\pi}=Z_{A}\left\langle 0\left|A_{0}(0)\right| \pi\right\rangle
$$

where $m_{\pi}$ is the pion mass, $A_{0}$ is the time component of the bare (lattice) axial current and $Z_{A}$ is its RC. These are specific examples in which operator renormalization is an essential step for passing from lattice to continuum physics. This renormalization is an inevitable requirement in those cases where the ME's of bare operators diverge in the continuum limit (e.g. the ME's of the $\Delta I=1 / 2$ four-fermion operators).

So far, three methods have been implemented to the computation of the $Z$ 's:

- Lattice Perturbation Theory (PT) [1]- 10]

- Non-perturbative use of Ward Identities (WI's), either on hadronic states [11-20 or on quark states 17, 19, 21].

- Non-perturbative (NP) renormalization on external quark and/or gluon states 22]-27.

Lattice PT can be applied to the calculation of finite and logarithmically divergent RC's'. The WI method is only applicable to finite RC's, whereas the NP one can be applied to all cases. The RC's, calculated to $\mathcal{O}\left(g_{0}^{2 n}\right)$ in PT, suffer from $\mathcal{O}\left(g_{0}^{2(n+1)}\right)$ systematic errors (in most cases $n=1$ ). On the other hand, non-perturbative determinations of the RC's (WI or NP method), are subject to $\mathcal{O}(a)$ systematic errors, since they are obtained from correlation functions computed at finite cutoff. Thus, physical quantities like those of eqs. (11) and (2), will suffer from $\mathcal{O}\left(g_{0}^{2(n+1)}\right)$ and $\mathcal{O}(a)$ systematic errors if the RC's are obtained in PT, and from $\mathcal{O}(a)$ errors if they are computed non

\footnotetext{
${ }^{1} \mathrm{PT}$ cannot be applied to power divergent RC's, because of the possible presence of finite non-perturbative contributions (see, for example, [25, 28] and references therein).
} 
perturbatively. Therefore WI or NP estimates of the RC's are clearly preferable in principle.

In recent years, there have been considerable efforts in refining the perturbative computation of the RC's. Boosted PT (BPT) has been extensively explored by Lepage and Mackenzie [29]. It consists in judicious choices of the expansion parameter (motivated by the behaviour of the resulting perturbative series, by tadpole resummation arguments and even by Mean Field Theory) which should bring the perturbative estimate of the RC's closer to their actual values. Even though the error remains $\mathcal{O}\left(g_{0}^{2(n+1)}\right)$, any of the several choices of BPT, proposed in [29], might improve the asymptotic convergence of the perturbative series.

On the non-perturbative side, Symanzik-type improved actions and operators have been constructed, in order to systematically reduce the finite cutoff error inherent in numerical computations. The implementation of the tree-level improved Clover action and operators [30, 31] reduces the discretization errors from $\mathcal{O}(a)$ to $\mathcal{O}\left(g_{0}^{2} a\right)$ (more precisely, it removes all $\mathcal{O}\left(a g_{0}^{2 n} \ln ^{n} a\right)$ systematic errors, which are effectively $\mathcal{O}(a)$ in the scaling limit, $\left.g_{0}^{2} \sim 1 / \ln a\right)$. Recently, a non-perturbative extension of the tree-level Clover improvement programme has been proposed in ref. [32], which corrects up to $\mathcal{O}\left(a^{2}\right)$ errors. The general proposal of these works is directly applicable in the chiral limit (with the exception of the improvement of the vector current which has also been implemented at non-zero quark mass; see ref. [33]). Another approach to the nonperturbative improvement programme, which can also be applied with finite values of the quark masses, can be found in in refs. [34, 35].

Regarding the RC's, the main achievement of the tree-level improvement programme has been the reduction of discretization errors from about $20 \%$ to about $5 \%$ in computations with light quark masses [15]. The situation is more problematic with heavy quark masses, say $a m \gtrsim 0.4$. Even with a tree-level improved action, the dominant $\mathcal{O}\left(g_{0}^{2} a m\right)$ error is appreciable. The most direct way of reducing these errors consists in reducing the lattice spacing (by going to larger $\beta$ values), but the increase in computational cost becomes prohibitive. Alternatively, one can try to reduce systematically these errors at fixed cutoff. The proposal of refs. [36] - [39] is such an effort. It consists in matching lattice quantities to their continuum counterparts at vanishing spatial momentum, ap, and large finite masses, $a m$. The aim is to eventually remove all am corrections, leaving predominantly $\mathcal{O}(a p)$ errors. This program has been explicitly carried out only in the free theory; we refer to it as the KLM approach. Speculations about higher order extensions can be found in [39].

This work is a thorough comparison of the various estimates (perturbative and nonperturbative) of the RC's of two-fermion lattice operators. We have tried to be exhaustive in investigating the numerous improvement proposals found in the literature. In particular:

- We have concentrated on the finite RC's of the vector and axial currents $\left(Z_{V}\right.$ and $Z_{A}$ ) and the finite ratio $Z_{S} / Z_{P}$ of the RC's of the scalar and pseudoscalar densities. 
- We have examined the influence of boosting to the 1-loop perturbative estimates of the above quantities. Several boosting recipes of ref. [29] have been applied. The "optimal" scales of the boosted running coupling (the $q^{*}$ of ref. [29]) has been obtained for $Z_{V}, Z_{A}$ and $Z_{S} / Z_{P}$. The $q^{*}$ values can be found in table 1 . We find that all recipes of $\mathrm{BPT}$ give the same results within a few per cent $(10 \%$ in the worst case). These results are summarized in table 2. Moreover, all BPT results differ from those obtained in SPT by up to $20 \%$. When compared with the RC's obtained from WI's (see table 3), BPT results are usually in better agreement than the SPT ones.

- The RC's $Z_{V}, Z_{A}$ and the ratio $Z_{S} / Z_{P}$ have subsequently been computed nonperturbatively, using the WI method, for several quark masses. Both the Wilson and the tree-level Clover actions have been implemented. Discretization errors at small quark mass, being $\mathcal{O}(a)$ tend to be about $20 \%-30 \%$ in the Wilson case. In the tree-level Clover case, being $\mathcal{O}\left(g_{0}^{2} a\right)$, these errors drop below $5 \%$. However, for masses $m a \gtrsim 0.4$, which are used in current lattice QCD simulations, they grow beyond $10 \%$. By extrapolating our data in the quark mass, we obtain extremely consistent Clover results for $Z_{V}$ and $Z_{A}$ at zero quark mass, shown in table 3. We have also checked that it is not possible to optimize these results by implementing different choices of improved operators, based on a different arbitrary admixture of the so-called $\not D$ and $m_{0}$ improving field rotations (see Subsection 5.2 for the definitions of these field rotations).

- In order to control discretization errors of increasing quark mass, we have applied the tree-level KLM matching prescription of refs. [36] - [39]. In the Wilson case, the large systematic error due to $\mathcal{O}(a m)$ effects is significantly reduced in some cases (see figure 1). There are cases, however, for which the KLM prescription fails to correct large $\mathcal{O}(\mathrm{am})$ effects (see figure 2). Moreover, a similar analysis, applied to the Clover case, revealed that KLM corrections fail to improve the situation. This is because the Clover correlation functions we have examined are characterized by large $\mathcal{O}\left(g_{0}^{2} a m\right)$ errors in the region of heavy quark masses. Thus, the KLM corrections, being of $\mathcal{O}\left(a^{2}\right)$, cannot absorb these discretization errors.

The present work is intended as a thorough comparison of the various improvement schemes which have been widely used in present-day, state-of-the-art lattice QCD phenomenology studies. As such, it has not dealt with the more recent non-perturbative Clover improvement of 33, 34, 35, which appears to be the most promising way to reduce finite cutoff effects.

The paper is organized as follows. In sect.2 we give some basic definitions. In sect. 3 we review the perturbative calculation of the RC's of two-fermion lattice operators. In subsec. 3.1 we discuss BPT and compare results for the RC's obtained from various boosting recipes. We also present the "optimal scales" $q^{*}$ of [29] for the operators of interest (some technical subtleties of this calculation can be found in the Appendix). In sect. 4 we review the well known vector and axial WI's on the lattice, with particular emphasis on the conditions under which they can be used to obtain the RC's. We 
have gathered in this section all WI's which are subsequently used in sects. 5 and 7 , where we discuss the practical implementation of the WI determination of RC's and present our results for $Z_{V}, Z_{A}$ and $Z_{S} / Z_{P}$. In sect. 6 we review the tree-level factors of the KLM-procedure for the Wilson correlation functions and derive their Clover counterparts. Finally, in sect. 8 we present our conclusion.

\section{Definitions and Generalities on RC's}

In this section we define the quantities of interest to this paper. Besides fixing our notation, we also review some general properties of the RC's of lattice operators, with particular attention to their dependence on the theory's parameters and renormalization scale.

We consider bilinear quark operators of the form:

$$
O_{\Gamma}^{a}(x)=\bar{\psi}(x) \Gamma \frac{\lambda^{a}}{2} \psi(x)
$$

where $\psi(x)$ is the quark field, $\Gamma$ denotes a generic Dirac matrix and $\lambda^{a} / 2$ are the generators of the $S U\left(N_{f}\right)$ flavour group in the fundamental representation $\left(a=1, \ldots, N_{f}^{2}-1\right)$. These generators satisfy:

$$
\begin{aligned}
& \operatorname{Tr}\left[\lambda^{a} \lambda^{b}\right]=2 \delta^{a b} \\
& {\left[\frac{\lambda^{f}}{2}, \frac{\lambda^{g}}{2}\right]=i f f g \frac{\lambda^{h}}{2}} \\
& \left\{\frac{\lambda^{f}}{2}, \frac{\lambda^{g}}{2}\right\}=d^{f g h} \frac{\lambda^{h}}{2}+\frac{\delta^{f g}}{N_{f}} I
\end{aligned}
$$

where $I$ represents the identity. Specific bilinear operators will be denoted according to their Lorentz group transformations: the scalar and pseudoscalar densities are denoted by $S^{a}(x)=\bar{\psi}(x) \frac{\lambda^{a}}{2} \psi(x)$ and $P^{a}(x)=\bar{\psi}(x) \frac{\lambda^{a}}{2} \gamma_{5} \psi(x)$ respectively, whereas the local vector and axial vector currents by $V_{\mu}^{a}(x)=\bar{\psi}(x) \frac{\lambda^{a}}{2} \gamma_{\mu} \psi(x)$ and $A_{\mu}^{a}(x)=$ $\bar{\psi}(x) \frac{\lambda^{a}}{2} \gamma_{\mu} \gamma_{5} \psi(x)$. Only such non-singlet bilinear quark operators will be considered in this paper. In the case of the vector and axial currents, we will also be using their point-split discretizations.

We will deal with operator insertions in $n$-point Green's functions, with the operator usually inserted at the origin. For example the insertion of $O_{\Gamma}$ in the 2-point Green's function is

$$
G_{O}\left(x_{1}, x_{2}\right)=\left\langle\psi\left(x_{1}\right) O_{\Gamma}^{a}(0) \bar{\psi}\left(x_{2}\right)\right\rangle
$$

In momentum space the insertion becomes

$$
G_{O}\left(p_{1}, p_{2}\right)=\int d x_{1} d x_{2} \exp \left(i p_{1} x_{1}\right) \exp \left(-i p_{2} x_{2}\right) G_{O}\left(x_{1}, x_{2}\right)
$$


and the corresponding amputated Green's function is given by

$$
\Lambda_{O}\left(p_{1}, p_{2}\right)=\mathcal{S}^{-1}\left(p_{1}\right) G_{O}\left(p_{1}, p_{2}\right) \mathcal{S}^{-1}\left(p_{2}\right)
$$

where $\mathcal{S}\left(x_{1}-x_{2}\right)=\left\langle\psi\left(x_{1}\right) \bar{\psi}\left(x_{2}\right)\right\rangle$ is the quark propagator and $\mathcal{S}(p)$ its momentum space counterpart. We will also be using Green's functions between external hadronic states. These are defined in an analogous fashion. For example, the 3-point correlation function $\left\langle P\left(x_{1}\right) V_{\mu}(0) P^{\dagger}\left(x_{2}\right)\right\rangle$ is a correlation function of three bilinear operators. From its Fourier transform in 3-dimensional space, and with the aid of the spectral representation formula at large euclidean time separations, the on-shell hadronic ME $\left\langle\pi\left(\vec{p}_{1}\right)\left|V_{\mu}\right| \pi\left(\vec{p}_{2}\right)\right\rangle$ can be readily extracted.

Everything defined so far is a bare quantity, which we assume to be defined in the lattice regularization?. We opt for the lattice regularization scheme proposed by Wilson which consists of a gluonic action 40,

$$
S_{g}=-\frac{6}{g_{0}^{2}} \sum_{P} \operatorname{Tr}\left[U_{P}+U_{P}^{\dagger}\right]
$$

and a fermionic part 41,

$$
\begin{aligned}
S_{f}= & -a^{4} \sum_{x, \mu} \frac{1}{2 a}\left[\bar{\psi}(x)\left(1-\gamma_{\mu}\right) U_{\mu}(x) \psi(x+\mu)+\bar{\psi}(x+\mu)\left(1+\gamma_{\mu}\right) U_{\mu}^{\dagger}(x) \psi(x)\right] \\
& +a^{4} \sum_{x} \bar{\psi}(x)\left(M_{0}+\frac{4}{a}\right) \psi(x)
\end{aligned}
$$

where, in standard notation, $U_{P}$ is the Wilson plaquette, $U_{\mu}(x)$ is the lattice gauge link and $g_{0}$ the bare coupling constant. The fermion field is a vector in flavour space. Its components will be denoted by $u, d, s$ etc, or by $\psi_{1}, \psi_{2}, \psi_{3}$, etc. according to notational convenience. The diagonal bare mass matrix is denoted by $M_{0}$ and its elements by $m_{01}, m_{02}, m_{03}$ etc. (or $m_{0 u}, m_{0 d}, m_{0 s}$ etc.). We use $m_{0}$ in the mass degenerate case. As is well known, for Wilson fermions, the quark mass, besides a multiplicative renormalization, is also subject to an additive one. Defining $m=m_{0}-m_{C}$, the renormalized mass will be given by:

$$
m_{R}=Z_{m} m=Z_{m}\left[m_{0}-m_{C}\right]
$$

with $Z_{m}$ the multiplicative RC. The chiral limit is then $m \rightarrow 0$; i.e. $m_{0} \rightarrow m_{C}$. At tree-level, $m_{C}=-4 / a$. For the diagonal elements of the matrix $M_{0}-m_{C}$ we will use either $m_{1}, m_{2}, m_{3}$ etc., or $m_{u}, m_{d}, m_{s}$ etc., according to notational convenience.

All operators studied in this work are subject to multiplicative renormalization; cases involving mixing of equal- or lower-dimensional operators (e.g. the four-fermion

\footnotetext{
${ }^{2}$ This means that the integrals of eq. (6) are really sums $\left(a^{8} \sum_{x_{1}, x_{2}}\right)$ which run over all lattice sites, labelled by $x_{1}, x_{2}$, etc. The use of integrals instead of sums and Dirac functions instead of Kronecker symbols is a question of notational convenience, which should be transparent.
} 
operators driving the $\Delta S=2$ or $\Delta I=1 / 2$ transitions) will not be considered. Thus, the renormalized operator is given by

$$
\hat{O}_{\Gamma}\left(g_{R}^{2}, m_{R}, \mu\right)=\lim _{a \rightarrow 0}\left[Z_{O}\left(g_{0}^{2}, a, m, \mu\right) O_{\Gamma}\left(g_{0}^{2}, m, a\right)\right]
$$

where $g_{R}$ is the renormalized gauge coupling and $\mu$ the renormalization scale. Note that in the bare quantities, the bare mass $m_{0}$ has been traded off for the more convenient (from the point of view of the chiral limit) subtracted mass $m$. A specific, if arbitrary, choice of renormalization scheme and scale are implied. Suppressing the dependence on couplings, masses, scales and cutoffs for notational simplicity, we list other relationships of interest between bare and renormalized quantities (denoted in general by "hats"): the renormalized quark propagator is

$$
\hat{\mathcal{S}}(p)=\lim _{a \rightarrow 0}\left[Z_{\psi} \mathcal{S}(p)\right]
$$

where $Z_{\psi}^{1 / 2}$ is the quark field RC. From the above definitions of $Z_{O}$ and $Z_{\psi}$, with the aid of eqs. (5) - (7), we find for the renormalized Green's functions:

$$
\begin{aligned}
& \hat{G}_{O}\left(p_{1}, p_{2}\right)=\lim _{a \rightarrow 0}\left[Z_{\psi} Z_{O} G_{O}\left(p_{1}, p_{2}\right)\right] \\
& \hat{\Lambda}_{O}\left(p_{1}, p_{2}\right)=\lim _{a \rightarrow 0}\left[Z_{\psi}^{-1} Z_{O} \Lambda_{O}\left(p_{1}, p_{2}\right)\right]
\end{aligned}
$$

Let us now examine the dependence of the RC's $Z_{O}$ on the bare parameters of the action. Multiplicative $Z_{O}$ 's are dimensionless quantities. Consequently, their functional dependence on the bare parameters and the cutoff can in principle be only of the form $Z_{O}\left(g_{0}^{2}, a m, a \mu\right)$. For the specific case of finite RC's (e.g. those of the vector and axial currents) there is no dependence on the renormalization scale $\mu$. In other words, the anomalous dimension is zero; this will be discussed in detail in the following section. Therefore, we are left with a possible dependence of the form $Z_{O}\left(g_{0}^{2}, a m\right)$. However, there can be no dependence of $Z_{O}$ on $a m$. Regular terms such as positive powers of $a m$, which may appear in the calculation of the bare Green's function $\Lambda_{O}$, will drop out of $Z_{O}$, since RC's are strictly defined in the continuum limit $a \rightarrow 0$; c.f. eq. (13). This leaves us with singular terms, such as $\ln (a m)$ and negative powers of am. But since we require the existence of the chiral limit $(m \rightarrow 0)$ in QCD, such a dependence must also be ruled out. This exhausts all possibilities, and renders $Z_{O}$ independent of $a m$. In conclusion, the finite RC's $Z_{V}, Z_{A}$ and the ratio $Z_{S} / Z_{P}$, examined in this paper, are of the form $Z_{O}\left(g_{0}^{2}\right) \cdot{ }^{3}$

\footnotetext{
${ }^{3}$ Dimensionless RC's which are not finite have the general dependence $Z_{O}\left(g_{0}^{2}, a m, a \mu\right)$. Provided that the renormalization scale $\mu$ is chosen to be greater then the quark masses $(\mu \gg m)$, there can be no dependence on am (same argument as above). The requirement of a well defined chiral limit allows only regular terms in $m / \mu$, which however have been assumed negligible. Thus, in this case the RC's have the form $Z_{O}\left(g_{0}^{2}, a \mu\right)$
} 


\section{Evaluation of RC's from Perturbation Theory}

In this section we review the basic concepts of the perturbative evaluation of the RC's of lattice operators and examine the proposals of ref. [29] which aims at the improvement of the convergence of the perturbative series in lattice PT.

The standard perturbative determination of the $\mathrm{RC} Z_{O}$ of a bilinear quark operator $O_{\Gamma}$ (flavour indices are suppressed, unless necessary) requires the calculation of the quark self- energy and the amputated Green function $\Lambda_{O}(p)$ of eq. (7) between external quark states, both at momentum $p_{\mu}$ (see, for example, [2]). Here we will work with the projected quark propagator $\Gamma_{\mathcal{S}}(p)$, and projected amputated Green function $\Gamma_{O}(p)$, defined as

$$
\begin{aligned}
& \Gamma_{\Sigma}(p)=\frac{-i}{48} \operatorname{Tr}\left[\gamma_{\mu} \frac{\partial \mathcal{S}(p)^{-1}}{\partial p_{\mu}}\right] \\
& \Gamma_{O}(p)=\frac{1}{12} \operatorname{Tr}\left[P_{O} \Lambda_{O}(p)\right]
\end{aligned}
$$

where the trace is over spin and colour indices and $P_{O}$ is the Dirac matrix which renders the tree-level value of $\Gamma_{O}(p)$ equal to unity (i.e. it projects out the nominal Dirac structure of the Green function $\left.\Lambda_{O}(p)\right)$ :

$$
\begin{aligned}
& P_{S}=I \quad ; \quad P_{P}=\gamma_{5} \\
& P_{V}=\frac{1}{4} \gamma_{\mu} \quad ; \quad P_{A}=\frac{1}{4} \gamma_{5} \gamma_{\mu}
\end{aligned}
$$

From eq. (12) we see that $\Gamma_{\Sigma}(p)$ renormalizes like

$$
\hat{\Gamma}_{\Sigma}(p)=\lim \left[Z_{\psi}^{-1} \Gamma_{\Sigma}(p)\right]
$$

The renormalization of $\Gamma_{O}(p)$ is identical to that of $\Lambda_{O}(p)$; c.f. eq(13):

$$
\hat{\Gamma}_{O}(p)=\lim \left[Z_{\psi}^{-1} Z_{O} \Lambda_{O}(p)\right]
$$

In the last two equations, the limit corresponds to the removal of the UV cutoff (e.g. $a \rightarrow 0$ on the lattice). The choice of $\Gamma_{\Sigma}(p)$ and $\Gamma_{O}(p)$, being scalar quantities, simplifies the discussion. Projected Green's functions have been implemented in recent works (e.g. ref. [22]). Another simplification consists in working with zero quark mass. This is justified, as we have shown in the previous section, by the lack of mass dependence of $Z_{O}$, in the limit $\mu \gg m$.

Upon passing from formal definitions of renormalized quantities (like eq. (17)) to their specific evaluation, we need to implement a regularization. The standard practice in continuum calculations consists in choosing a Dimensional Regularization $(D R)$ scheme, such as Naive Dimensional Regularization $(N D R)$, 't Hooft - Veltman $(H V)$ etc. The expression for $\Gamma_{O}$, evaluated at 1-loop PT, can be written in the general form:

$$
\Gamma_{O}^{D R}\left(p, g_{0}, \epsilon\right)=\left[1+\frac{g_{0}(\mu)^{2}}{(4 \pi)^{2}}\left(\gamma_{\Gamma} \frac{1}{\hat{\epsilon}}-\gamma_{\Gamma} \ln (p / \mu)^{2}+C_{\Gamma}^{D R}\right)\right]+\mathcal{O}(\epsilon)
$$


where $\gamma_{\Gamma}$ is an anomalous dimension and $C_{\Gamma}^{D R}$ is a finite constant, which depends on the regularization scheme and the gauge chosen. The factor $1 / \hat{\epsilon}$ in eq. (18) is an abbreviation for

$$
\frac{1}{\hat{\epsilon}}=\frac{1}{\epsilon}+\ln (4 \pi)-\gamma_{E}
$$

where $\epsilon=(4-D) / 2$ and $\gamma_{E}$ stands for Euler's constant. In $D R$ we work in $D$ dimensions, where the original bare coupling $g_{0}$ has dimension $\epsilon$. Here the scale $\mu$ is introduced to render the bare coupling $g_{0}(\mu)$ dimensionless. The $\mu$-dependence of the r.h.s. of eq. (18) is apparent.

In order to renormalize $\Gamma_{O}$, we are in principle free to impose any renormalization scheme. The $\overline{\mathrm{MS}}$ scheme, at 1-loop, amounts to removing the $1 / \hat{\epsilon}$ divergence. Since the $\mathrm{RC}$ of the projected Green function $\Gamma_{O}$ is given by $Z_{\Gamma}=Z_{\psi}^{-1} Z_{O}$ (see eq. (17)), this implies for $Z_{\Gamma}$ the value

$$
Z_{\Gamma}^{\overline{\mathrm{MS}}, D R}\left(g_{0}(\mu), \epsilon\right)=1-\frac{g_{0}^{2}(\mu)}{(4 \pi)^{2}} \gamma_{\Gamma} \frac{1}{\hat{\epsilon}}
$$

Consequently, the renormalized Green function is given by

$$
\begin{gathered}
\hat{\Gamma}_{O}^{\overline{\mathrm{MS}}}\left(p, g_{\overline{\mathrm{MS}}}(\mu), \mu\right)=\lim _{\epsilon \rightarrow 0}\left[Z_{\Gamma}^{\overline{\mathrm{MS}}, D R}\left(g_{0}(\mu), \epsilon\right) \Gamma_{O}^{D R}\left(p, g_{0}, \epsilon\right)\right]= \\
=\left[1+\frac{g_{\overline{\mathrm{MS}}}^{2}(\mu)}{(4 \pi)^{2}}\left(-\gamma_{\Gamma} \ln (p / \mu)^{2}+C_{\Gamma}^{D R}\right)\right]
\end{gathered}
$$

where, to this order, we are free to replace $g_{0}$ by $g_{\overline{\mathrm{MS}}}(\mu)$, the $\overline{\mathrm{MS}}$ renormalized coupling constant.

The same calculation can be repeated on the lattice. Now the UV cutoff is provided by the inverse finite lattice spacing $a^{-1}$ and thus the 1-loop calculation yields:

$$
\Gamma_{O}^{L A T}\left(p, g_{0}(a), a\right)=\left[1+\frac{g_{0}(a)^{2}}{(4 \pi)^{2}}\left(-\gamma_{\Gamma} \ln (p a)^{2}+C_{\Gamma}^{L A T}\right)\right]+\mathcal{O}(a)
$$

where $g_{0}$ is the bare coupling of the lattice action. Note that at 1-loop the anomalous dimension $\gamma_{\Gamma}$ is regularization independent.

The renormalization scheme can again be chosen at will; often the $\overline{\mathrm{MS}}$ is chosen also on the lattice. This seemingly unnatural choice (the $\overline{\mathrm{MS}}$ is closely linked to continuum DR) has a few advantages. For example, ME's of effective Hamiltonians, once calculated non-perturbatively on the lattice, must be renormalized and combined with perturbatively calculated Wilson coefficients, in order to obtain physical amplitudes. The renormalization-group invariance of these amplitudes is guaranteed only if the Wilson coefficients and the RC's are calculated in the same renormalization scheme. Since the former are often known in the $\overline{\mathrm{MS}}$ scheme, this scheme is also preferred for the calculation of the latter. This choice then corresponds to the requirement

$$
\hat{\Gamma}_{O}^{\overline{\mathrm{MS}}}\left(p, g_{\overline{\mathrm{MS}}}(\mu), \mu\right)=\lim _{a \rightarrow 0}\left[Z_{\Gamma}^{\overline{\mathrm{MS}}, L A T}\left(\mu a, g_{0}(a)\right) \Gamma_{O}^{L A T}\left(p, g_{0}(a), a\right)\right]
$$


where again the lattice coupling $g_{0}(a)$ should be traded for the $\overline{\mathrm{MS}}$ renormalized coupling constant $g_{\overline{\mathrm{MS}}}(\mu)$. This point of principle is of limited relevance for a 1-loop calculation. From eqs. (21), (22) and (23), the following RC is obtained:

$$
Z_{\Gamma}^{\overline{\mathrm{MS}}, L A T}\left(\mu a, g_{0}(a)\right)=1+\frac{g_{0}(a)^{2}}{(4 \pi)^{2}}\left[\gamma_{\Gamma} \ln (\mu a)^{2}+C_{\Gamma}^{D R}-C_{\Gamma}^{L A T}\right]
$$

We now recall that the $\mathrm{RC}$ of the amputated vertex $\Gamma_{O}$ is $Z_{\Gamma}=Z_{\psi}^{-1} Z_{O}$. The $\mathrm{RC}$ of the quark field $Z_{\psi}$ can be calculated, from the renormalization of the quark propagator $\mathcal{S}(p)$, with an analogous procedure (c.f. eq. (16)). The result is

$$
Z_{\psi}^{\overline{\mathrm{MS}}, L A T}\left(\mu a, g_{0}(a)\right)=1+\frac{g_{0}(a)^{2}}{(4 \pi)^{2}}\left[\gamma_{\Sigma} \ln (\mu a)^{2}+C_{\Sigma}^{D R}-C_{\Sigma}^{L A T}\right]
$$

Combining eqs. (24) and (25) we obtain

$$
Z_{O}^{\overline{\mathrm{MS}}, L A T}\left(\mu a, g_{0}(a)\right)=1+\frac{g_{0}(a)^{2}}{(4 \pi)^{2}}\left[\gamma_{O} \ln (\mu a)^{2}+\Delta_{\Gamma}+\Delta_{\Sigma}\right]
$$

where

$$
\begin{aligned}
\gamma_{O} & =\gamma_{\Gamma}+\gamma_{\Sigma} \\
\Delta_{\Gamma} & =C_{\Gamma}^{D R}-C_{\Gamma}^{L A T} \\
\Delta_{\Sigma} & =C_{\Sigma}^{D R}-C_{\Sigma}^{L A T}
\end{aligned}
$$

It is this RC (with this choice of renormalization condition) which is usually denoted by $Z_{O}$ in lattice PT calculations. The dependence of $Z_{O}^{\overline{\mathrm{MS}}, L A T}$ on the coefficients $C_{\Gamma}^{D R}$ and $C_{\Sigma}^{D R}$ comes from the choice of the $\overline{\mathrm{MS}}$ renormalization condition (see eqs. (21) and (23)) whereas its dependence on $C_{\Gamma}^{L A T}$ and $C_{\Sigma}^{L A T}$ from the lattice regularization (see eq. (22)). Two perturbative calculations are thus necessary, one in the continuum for the $C^{D R}$ 's and one on the lattice for $C^{L A T}$ 's. The presence of the $C^{D R}$ 's on the r.h.s. of eq. (24) is sometimes called regularization dependence of the renormalization scheme [42, 43]. The reader is referred to ref. [2] for the explicit calculation of the RC's of several bilinear operators with the Wilson action. The corresponding results for the Clover action appeared in [7, 8].

The PT method is applicable to finite and logarithmically divergent operators, the renormalization of which does not require a subtraction of lower dimensional operators. Model examples of operators with logarithmically divergent multiplicative RC's are the scalar density $S(x)=\bar{\psi}_{1}(x) \psi_{2}(x)$ and the pseudoscalar density $P(x)=\bar{\psi}_{1}(x) \gamma_{5} \psi_{2}(x)$. These are the cases best suited to our general discussion so far.

The RC's of the vector current $V_{\mu}^{a}(x)$ and the axial current $A_{\mu}^{a}(x)$ are also calculable in PT on similar lines. Being (partially) conserved quantities however, they have some peculiarities. Their (partial) conservation guarantees zero anomalous dimension (i.e $\gamma_{V}=0$ and $\gamma_{A}=0$ ). This result is regularization independent (e.g. valid both in 
the continuum $(D R)$ and the lattice $(L A T)$. It implies that the current renormalization constants are finite, scale independent quantities. Moreover, the following useful properties must be taken into consideration:

- The vector current conservation survives the continuum DR. Thus it satisfies a WI which implies that $Z_{V}^{\overline{\mathrm{MS}}, D R}=1$. This is an exact result, valid at all orders of PT. Therefore, at 1-loop $V_{\mu}^{a}(x)$ has zero finite constant; i.e. $C_{V}^{D R}+C_{\Sigma}^{D R}=0$.

- As we will see in the next section, the local lattice vector current is not a conserved quantity. It has a non-vanishing 1-loop contribution (i.e. $C_{V}^{L A T}+C_{\Sigma}^{L A T} \neq 0$ ). In other words, $Z_{V}^{\overline{\mathrm{MS}}, L A T}\left(g_{0}^{2}\right) \neq 1$.

- We will also show in the next section that on the lattice one can define an extended conserved vector current, $\widetilde{V}_{\mu}^{a}(x)$, which is also trivially renormalized with $Z_{\widetilde{V}}^{\overline{\mathrm{MS}}, L A T}=1$. Thus, also for this current we have $C_{\tilde{V}}^{L A T}+C_{\Sigma}^{L A T}=0$.

- For the axial current the argument is almost the same. However, some further peculiarities arise due to the breaking of the chiral symmetry by the regularization. In the continuum, the finite 1-loop contribution to the renormalization constant may or may not vanish, depending on the detailed choice of the regularization scheme. Thus, for $N D R$ we have $C_{A}^{N D R}+C_{\Sigma}^{N D R}=0$, implying $Z_{A}^{\overline{\mathrm{MS}}, N D R}=1$. On the other hand, with $H V$ for example, we have $C_{A}^{H V}+C_{\Sigma}^{H V} \neq 0$, implying $Z_{A}^{\overline{\mathrm{MS}}, H V}\left(g_{0}^{2}\right) \neq 1$. In the present work, we will always imply $N D R$ when referring to $D R$.

- We will show in the next section that the lattice regularization breaks chiral symmetry. Thus on the lattice $C_{A}^{L A T}+C_{\Sigma}^{L A T} \neq 0$ (for any definition of the bare lattice current, local or extended). In other words, $Z_{A}^{\overline{\mathrm{MS}}, L A T}\left(g_{0}^{2}\right) \neq 1$

We are also interested in the finite ratio $Z_{S} / Z_{P}$. Its finiteness relies on WI's (see next section) and implies that $\gamma_{S}=\gamma_{P}$. With the specific choice of $N D R$ as the continuum dimensional regularization, we also have $C_{S}^{N D R}=C_{P}^{N D R}$.

\subsection{Boosted Improvement of the PT Estimates of RC's}

The perturbative calculation outlined above is only approximate, because of the truncation of the perturbative series. At present, most lattice RC's are only known in PT at 1-loop. Thus, they suffer from $\mathcal{O}\left(g_{0}^{4}\right)$ systematic errors. These errors must be born in mind when comparing the RC's, calculated in PT, to their non-perturbative estimates. In this subsection we discuss these systematic errors and comment on the proposals for improving the convergence of the perturbative series of ref. [29]. These proposals are known as Boosted Perturbation Theory (BPT).

We have already pointed out that, in principle, at 1-loop there is an ambiguity in the choice of couplings $\left(g_{0}(a), g_{0}(\mu), g_{\overline{\mathrm{MS}}}(\mu)\right)$ to be used in eq. (24). This ambiguity is lifted in (and up to) 2-loop PT. At 1-loop however, a judicious choice of expansion 
parameter may be important in practice. The lattice coupling $g_{0}^{2}(a)$ has been shown to be a bad expansion parameter in ref. [29], where several improved (boosted) couplings have been proposed, and several observables have been calculated in PT with these couplings.

In the specific case of $Z_{O}$, the Standard Perturbation Theory (SPT) result is given as a series in the bare (lattice) coupling $g_{0}$

$$
Z_{O}\left(a \mu, g_{0}^{2}(a)\right)=1+C_{1}(a \mu) g_{0}^{2}(a)+C_{2}(a \mu) g_{0}^{4}(a)+\ldots
$$

To first order, the above equation reduces to eq. (26), with $C_{1}(a \mu)=\left[\gamma_{O} \ln (a \mu)^{2}+\right.$ $\left.\Delta_{\Gamma}+\Delta_{\Sigma}\right] /(4 \pi)^{2}$. According to [29], perturbative series have a better behaviour if expressed in terms of a renormalized coupling $g_{V}^{2}\left(q^{*}\right)$ at a suitable scale $q^{*}$ (to be specified shortly):

$$
Z_{O}\left(a \mu, g_{V}^{2}\left(q^{*}\right)\right)=1+C_{1}(a \mu) g_{V}^{2}\left(q^{*}\right)+\widetilde{C}_{2}\left(a \mu, a q^{*}\right) g_{V}^{4}\left(q^{*}\right)+\ldots
$$

The claim is that the perturbative estimate obtained from the boosted series, truncated at low orders, is closer to the non-perturbative result than its SPT counterpart.

The renormalized coupling $g_{V}(q)$ in eq. (29) can be chosen at will. In ref. [29] it is suggested to define it from the heavy quark potential $V(q)$ at momentum transfer $q$ :

$$
V(q)=-\frac{4 g_{V}^{2}(q)}{3 q^{2}}
$$

Moreover, according to [29] and [44], the scale $q^{*}$, which is most appropriate to the expansion (29) of $Z_{O}$, is fixed by:

$$
\ln \left(q^{* 2}\right)=\frac{\int d^{4} q f(q, a \mu) \ln \left(q^{2}\right)}{\int d^{4} q f(q, a \mu)}
$$

where $f(q, a \mu)$ is the function entering in the loop integral which defines the constant $C_{1}(a \mu)$ of eq. (29): $C_{1}(a \mu)=\int d^{4} q f(q, a \mu)$. f From the above expression we readily see that in general $q^{*}$ depends on the renormalization scale $\mu$. Here, however, we are only interested in the finite quantities $Z_{V}, Z_{A}$ and $Z_{S} / Z_{P}$, for which the $C_{i}$ 's of eq. (28) are $\mu$-independent. Consequently, also $q^{*}$ does not depend on $\mu$.

The connection between the bare coupling $g_{0}^{2}$ and the renormalized coupling $g_{V}^{2}$ defined in eq. (30) is obtained by calculating the heavy quark potential in lattice PT. At lowest order one finds

$$
g_{V}^{2}(q)=g_{0}^{2}(a)\left[1-g_{0}^{2}(a) 2 \beta_{0} \ln (a q / c)+\mathcal{O}\left(g_{0}^{4}(a)\right)\right]
$$

${ }^{4}$ Here we are dealing with multiplicative renormalization, for which the above recipe is fairly non ambiguous. However, if the operator mixes with others under renormalization, the recipe is ambiguous, since one can either evaluate all mixing constants at the same scale $q^{*}$ or obtain each mixing constant at a separate scale. 
where $\beta_{0}=11 /(4 \pi)^{2}$ and $c$ is given by:

$$
c=\pi \exp \left(\frac{4.702}{8 \pi \beta_{0}}\right)
$$

There are several possibilities, suggested in [29], for extracting $g_{V}^{2}\left(q^{*}\right)$ from eq. (32). They can be classified as follows:

1. Purely Perturbative Boosting [PPB]: This method consists in using eq. (32) in order to identify the couplings at scale $q=c / a$ :

$$
g_{V}^{2}(c / a)=g_{0}^{2}(a)+\mathcal{O}\left(g_{0}^{4}\right)
$$

Then one can perform 2-loop Renormalization Group (RG) running of $g_{V}^{2}(c / a)$ down to the scale $q^{*}$ in the standard fashion:

$$
\frac{1}{g_{V}^{2}\left(q^{*}\right)}=\frac{1}{g_{V}^{2}(c / a)}+2 \beta_{0} \ln \left(\frac{a q^{*}}{c}\right)+\frac{\beta_{1}}{\beta_{0}} \ln \left(\frac{g_{V}^{2}(c / a)}{g_{V}^{2}\left(q^{*}\right)}\right)
$$

with $\beta_{1}=102 /(4 \pi)^{4}$. Thus, having calculated $q^{*}$ (from eq. (31)) and $g_{V}^{2}\left(q^{*}\right)$, we derive $Z_{O}$ from eq. (29). We call this estimate PPB.

2. Monte Carlo - Perturbative Boosting [MC-PB(ln $\square)]$ : Again this method is based on the relationship between $g_{V}^{2}$ and $g_{0}^{2}$ of eq. (32). But now the boosted coupling constant is extracted from the non-perturbative (Monte Carlo) value of a short distance quantity, such as the average plaquette 455. The logarithm of the plaquette, known as a perturbative series in $g_{0}^{2}$, is expressed, with the aid of eq. (32), as a series in $g_{V}^{2}$. The leading coefficient of this series is an integral of the kind $\int d^{4} q f_{\square}(q)$, which gives rise, through the criterion of eq. (31), to the scale $q_{\square}^{*}$, appropriate for this expansion. From ref. [29] we have $q_{\square}^{*}=3.41 / a$ and the expansion:

$$
\ln \left(\frac{1}{3} \operatorname{Tr}\left\langle U_{\square}\right\rangle\right)=-\frac{1}{3} g_{V}^{2}\left(q_{\square}^{*}\right)\left[1-9.46 \cdot 10^{-2} g_{V}^{2}\left(q_{\square}^{*}\right)+\mathcal{O}\left(g_{V}^{4}\right)\right]
$$

The series of the plaquette's logarithm has been preferred to that of the plaquette itself because, as claimed in ref. [29], its perturbative expansion is better behaving. Using the Monte Carlo result for the average plaquette, eq. (36) is solved for $g_{V}^{2}\left(q_{\square}^{*}\right)$. 2-loops RG running subsequently yields the desired $g_{V}^{2}\left(q^{*}\right)$. With this value for $g_{V}^{2}\left(q^{*}\right)$, we derive $Z_{O}$ from eq. (29). We call this method MC-PB(ln $\left.\square\right)$.

3. Monte Carlo- Perturbative Boosting [MC-PB( $\square)]$ : Yet another proposal of [29] consists in combining eqs. (32), at the scale $q=\pi / a$, and the perturbative expansion of the plaquette

$$
\frac{1}{3} \operatorname{Tr}\left\langle U_{\square}\right\rangle=1-\frac{1}{3} g_{V}^{2}(\pi / a)+8.71 \cdot 10^{-2} g_{V}^{4}(\pi / a)+\mathcal{O}\left(g_{V}^{6}\right)
$$

to obtain:

$$
g_{V}^{2}(\pi / a)\left[1-0.513 \frac{1}{4 \pi} g_{V}^{2}(\pi / a)+\mathcal{O}\left(g_{V}^{4}\right)\right]=\frac{g_{0}^{2}(a)}{\frac{1}{3} \operatorname{Tr}\left\langle U_{\square}\right\rangle}
$$




\begin{tabular}{|c|c|c|c|c|c|}
\hline \multicolumn{3}{|c|}{ Wilson } & \multicolumn{3}{c|}{ Clover } \\
\hline$a q^{*}\left(Z_{V}\right)$ & $a q^{*}\left(Z_{A}\right)$ & $a q^{*}\left(Z_{S} / Z_{P}\right)$ & $a q^{*}\left(Z_{V}\right)$ & $a q^{*}\left(Z_{A}\right)$ & $a q^{*}\left(Z_{S} / Z_{P}\right)$ \\
\hline 2.4 & 2.6 & 1.9 & 2.7 & 1.2 & 3.2 \\
\hline
\end{tabular}

Table 1: The values of the scale aq*, characteristic of the boosted perturbative evaluation of $Z_{V}, Z_{A}$ and $Z_{S} / Z_{P}$. The scales are shown for both the Wilson and Clover actions.

Once $g_{V}^{2}(\pi / a)$ is obtained from the above expression, two loop RG running yields $g_{V}^{2}\left(q^{*}\right)$ and subsequently $Z_{O}$. We call this estimate MC-PB( $\left.\square\right)$.

4. Naive Boosted Perturbation Theory [NBPT]: A particularly simple choice of boosted coupling [45] is $\tilde{g}^{2}=g_{0}^{2} /\left(\frac{1}{3} \operatorname{Tr}\left\langle U_{\square}\right\rangle\right)$ and the subsequent substitution of $g_{0}$ by $\tilde{g}^{2}$ in eq. (24). This recipe (a simplification of eq. (38)) is denoted by NBPT. It has been implemented, for example, in refs. [46] and [47]. As argued in ref. [29], Mean Field arguments can also be used in order to support this prescription.

Having classified the various boostings, we now comment on them. All these methods should be in principle equivalent to lowest order in PT. From the field theoretic point of view, $\mathrm{PPB}$ is a perfectly legitimate operation, as it amounts to a different choice of expansion parameter. The two MC-PB prescriptions may be even superior to PPB in practice, since they possibly incorporate some non-perturbative and higher order effects. In order to implement these recipes to the perturbative evaluation of the RC's of interest, we have calculated the appropriate scale $q^{*}$; this is different for each RC. The values of $q^{*}$ for $Z_{V}, Z_{A}$ and $Z_{S} / Z_{P}$ are collected in table 1. Some technical details concerning their evaluation are gathered in the Appendix.

In table 2, we collect the values of $Z_{O}$, obtained from the various boosting recipes listed above. We see that the variation of the boosted coupling, due to the choice of boosting recipe, does not exceed $20 \%$, and that of the various RC's is about $10 \%$. These variations reflect the systematic error introduced by the truncation of the perturbative series. In the next section, we will compare boosted results to the ones obtained non perturbatively from WI's. For this comparison we will use the MC-PB $(\ln \square)$ prescription which is considered the optimal choice by the authors of ref. [29]. As will be shown in table 3, such a comparison reveals a general trend for BPT to reduce the magnitude of the $\mathcal{O}\left(g_{0}^{4}\right)$ systematic error. However, in some cases this error remains appreciable (more than 10\%).

\section{Lattice WI's with Wilson Fermions}

In this section we review the WI's which follow from the chiral flavour symmetry of the QCD action. They allow to compute the finite RC's of lattice operators in a non-perturbative way. Several properties of these RC's can be derived from the WI's. Although the results presented here are well known (see [48, 49]), we give particular emphasis to the conditions (e.g. continuum limit, chiral limit etc.) under which these results are exact. This is essential to the understanding of the sources of systematic 


\begin{tabular}{|c|cc|cc|cc|}
\hline \multicolumn{7}{|c|}{ Wilson - $\beta=6.0$} \\
\hline & $g^{2}$ & $Z_{V}$ & $g^{2}$ & $Z_{A}$ & $g^{2}$ & $Z_{S} / Z_{P}$ \\
\hline SPT & 1.00 & 0.83 & 1.00 & 0.87 & 1.00 & 1.10 \\
PPB & 1.81 & 0.69 & 1.77 & 0.76 & 1.93 & 1.25 \\
MC-PB(ln $\square)$ & 2.14 & 0.63 & 2.08 & 0.72 & 2.32 & 1.34 \\
MC-PB( $\square)$ & 1.97 & 0.66 & 1.92 & 0.74 & 2.13 & 1.29 \\
NBPT & 1.68 & 0.71 & 1.68 & 0.77 & 1.68 & 1.20 \\
\hline \multicolumn{7}{|c|}{ Clover - $\beta=6.0$} \\
\hline SPT & $g^{2}$ & $Z_{V}$ & $g^{2}$ & $Z_{A}$ & $g^{2}$ & $Z_{S} / Z_{P}$ \\
PPB & 1.00 & 0.90 & 1.00 & 0.98 & 1.00 & 1.20 \\
MC-PB(ln $\square)$ & 1.75 & 0.83 & 2.25 & 0.96 & 1.67 & 1.42 \\
NBPT & 2.05 & 0.80 & 2.81 & 0.95 & 1.95 & 1.54 \\
& 1.90 & 0.81 & 2.52 & 0.96 & 1.81 & 1.48 \\
\hline
\end{tabular}

Table 2: Wilson and Clover action RC's obtained with different boosting procedures (see text for notation). The $R C$ 's are shown at $\beta=6.0$.

error in practical applications of the WI's, which form the basis of our analysis. The reader familiar with refs. [48, 49]) may skip this section.

We consider local $S U_{L}\left(N_{f}\right) \times S U_{R}\left(N_{f}\right)$ chiral transformations of the fermionic fields:

$$
\begin{aligned}
& \delta \psi(x)=i\left[\alpha_{V}^{a}(x) \frac{\lambda^{a}}{2}+\alpha_{A}^{a}(x) \frac{\lambda^{a}}{2} \gamma_{5}\right] \psi(x) \\
& \delta \bar{\psi}(x)=-i \bar{\psi}(x)\left[\alpha_{V}^{a}(x) \frac{\lambda^{a}}{2}-\alpha_{A}^{a}(x) \frac{\lambda^{a}}{2} \gamma_{5}\right]
\end{aligned}
$$

and examine the WI's derived separately in the vector and axial cases.

\subsection{Vector WI's on the Lattice}

With degenerate quark masses (i.e. $M_{0}$ in eq. (9) proportional to the unit matrix) global vector transformations are a symmetry of the action. From the corresponding local transformations (eqs. (39) with $\alpha_{A}^{a}=0$ ) the following vector WI can be derived 48 :

$$
\begin{aligned}
i\left\langle\frac{\delta O\left(x_{1}, \ldots, x_{n}\right)}{\delta \alpha_{V}^{a}(x)}\right\rangle= & a^{4} \sum_{\mu} \nabla_{x}^{\mu}\left\langle O\left(x_{1}, \ldots, x_{n}\right) \widetilde{V}_{\mu}^{a}(x)\right\rangle+ \\
& +a^{4}\left\langle O\left(x_{1}, \ldots, x_{n}\right) \bar{\psi}(x)\left[\frac{\lambda^{a}}{2}, M_{0}\right] \psi(x)\right\rangle
\end{aligned}
$$

where $O\left(x_{1}, \ldots, x_{n}\right)$ is any multi-local operator consisting of quark and gluon fields at different space-time points $\left(x_{1} \neq x_{2} \neq \ldots x_{n}\right) . \quad a \nabla_{x}^{\mu} f(x)=(f(x)-f(x-\mu))$ is an 
asymmetric lattice derivative, and

$$
\begin{aligned}
\tilde{V}_{\mu}^{a}(x)= & \frac{1}{2}\left[\bar{\psi}(x)\left(\gamma_{\mu}-1\right) U_{\mu}(x) \frac{\lambda^{a}}{2} \psi(x+\mu)+\right. \\
& \left.+\bar{\psi}(x+\mu)\left(\gamma_{\mu}+1\right) U_{\mu}^{\dagger}(x) \frac{\lambda^{a}}{2} \psi(x)\right]
\end{aligned}
$$

is a point-split vector current. By keeping the point $x$ separate from the points $x_{1}, \ldots, x_{n}$ and by taking the limit of degenerate bare quark masses, we see that eq. (40) leads to the conservation of the point split lattice vector current, $\nabla_{x}^{\mu} \widetilde{V}_{\mu}^{a}(x)=0$. This also implies that the standard local vector current $V_{\mu}^{a}(x)=\bar{\psi}(x) \frac{\lambda}{2} \gamma_{\mu} \psi(x)$ is not conserved on the lattice.

For the specific choice $O\left(x_{1}, x_{2}\right)=\psi\left(x_{1}\right) \bar{\psi}\left(x_{2}\right)$, the vector WI (40) becomes

$$
\begin{array}{r}
\sum_{\mu} \nabla_{x}^{\mu}\left\langle\psi\left(x_{1}\right) \widetilde{V}_{\mu}^{a}(x) \bar{\psi}\left(x_{2}\right)\right\rangle=\left\langle\psi\left(x_{1}\right) \bar{\psi}(x)\left[M_{0}, \frac{\lambda^{a}}{2}\right] \psi(x) \bar{\psi}\left(x_{2}\right)\right\rangle+ \\
+\delta\left(x_{2}-x\right)\left\langle\psi\left(x_{1}\right) \bar{\psi}\left(x_{2}\right)\right\rangle \frac{\lambda^{a}}{2}-\delta\left(x_{1}-x\right) \frac{\lambda^{a}}{2}\left\langle\psi\left(x_{1}\right) \bar{\psi}\left(x_{2}\right)\right\rangle
\end{array}
$$

Let us consider the case of degenerate quark masses $\left(M_{0} \propto 1\right)$ and place the vector current at the origin $(x=0)$. By Fourier - transforming the above equation and amputating the corresponding Green's function $G_{\widetilde{V}}^{\mu, a}=\left\langle\psi\left(x_{1}\right) \widetilde{V}^{\mu, a}(0) \bar{\psi}\left(x_{2}\right)\right\rangle$ (c.f. eqs. (5), (6) and (7)), we obtain

$$
\sum_{\mu}\left[\frac{1-\exp \left(-i a q_{\mu}\right)}{a}\right] \Lambda_{\widetilde{V}}^{\mu, a}\left(p+\frac{q}{2}, p-\frac{q}{2}\right)=-\mathcal{S}\left(p+\frac{q}{2}\right)^{-1} \frac{\lambda^{a}}{2}+\frac{\lambda^{a}}{2} \mathcal{S}\left(p-\frac{q}{2}\right)^{-1}
$$

This is the lattice version of the vector WI. In the continuum limit, it is a formal expression relating bare quantities which are divergent. In order to render them meaningful, it is essential to go over to renormalized expressions. With the RC's strictly defined in the limit of vanishing cutoff, we express the bare quantities of eq. (43) in terms of their renormalized counterparts, given in eqs. (12) and (13). Thus, in the limit $a \rightarrow 0$, we obtain:

$$
\sum_{\mu} i q_{\mu} Z_{\widetilde{V}}^{-1} \hat{\Lambda}_{V}^{\mu, a}\left(p+\frac{q}{2}, p-\frac{q}{2}\right)=-\hat{\mathcal{S}}\left(p+\frac{q}{2}\right)^{-1} \frac{\lambda^{a}}{2}+\frac{\lambda^{a}}{2} \hat{\mathcal{S}}\left(p-\frac{q}{2}\right)^{-1}
$$

However, the definitions of the RC's of eqs. (12) and (13), used to derive the above result, are only formal. A concrete definition implies the choice of a renormalization scheme. In the case of the vector current, it is crucial to impose that the renormalized operator $\hat{V}_{\mu}^{a}$ satisfy the nominal WI of the continuum theory. From this requirement on eq. (44) we obtain that the $\mathrm{RC}$ of the point split conserved vector current (41) is [48, 49]:

$$
Z_{\widetilde{V}}=1
$$


This result, which is exact in the limit $a \rightarrow 0$, guarantees a proper definition of the vector charge and the validity of current algebra. Thus, the vector symmetry of the theory survives renormalization.

The local vector current $V_{\mu}^{a}(x)=\bar{\psi}(x) \frac{\lambda^{a}}{2} \gamma_{\mu} \psi(x)$ is not conserved on the lattice. However, it can be shown that its ME's differ from those of the conserved current by finite contributions. The argument goes as follows: first we express the the conserved current as

$$
\widetilde{V}_{\mu}^{a}(x)=V_{\mu}^{a}(x)+\frac{a}{2}\left[\bar{\psi}(x)\left(\gamma_{\mu}-1\right) \frac{\lambda^{a}}{2} \overrightarrow{\mathcal{D}}_{\mu} \psi(x)+\bar{\psi}(x) \overleftarrow{\mathcal{D}}_{\mu}\left(\gamma_{\mu}+1\right) \frac{\lambda^{a}}{2} \psi(x)\right]
$$

where we have used the lattice asymmetric covariant derivatives

$$
\begin{aligned}
& a \overrightarrow{\mathcal{D}}_{\mu} \psi(x)=U_{\mu}(x) \psi(x+\mu)-\psi(x) \\
& a \bar{\psi}(x) \overleftarrow{\mathcal{D}}_{\mu}=\bar{\psi}(x+\mu) U_{\mu}^{\dagger}(x)-\bar{\psi}(x)
\end{aligned}
$$

The second term on the r.h.s. of eq. (46) is a dimension-4 operator (we call it $\Delta_{\mu}^{a}$ ) multiplied by the lattice spacing. For definitiveness, we now consider the Green's function $\Lambda_{\widetilde{V}}(p)$ of eq. (7). From eq. (46) it follows that

$$
\Lambda_{\widetilde{V}}(p)=\Lambda_{V}(p)+a \Lambda_{\Delta}(p)
$$

The term $a \Lambda_{\Delta}(p)$ vanishes at tree-level in the naive continuum limit. Beyond tree-level, however, this term contributes, due to power divergences induced by the mixing with lower dimensional operators. The mixing with operators of equal dimension gives at most logarithmic divergences $\ln (a p)$; thus in the continuum limit such contributions vanish like $a \ln (a p)$. As far as lower dimensional operators are concerned, $\Delta_{\mu}^{a}$ only mixes with the dimension- 3 vector current $V_{\mu}^{a}$. This gives rise to power divergences $a^{-1}$, without logarithms . Thus, the term $a \Lambda_{\Delta}$ gives finite contributions, which combine with the renormalization of $\Lambda_{V}$ to give the known non-renormalization exact result $Z_{\widetilde{V}}=1$ of the l.h.s. of eq. (48). Consequently, the term $\Lambda_{V}$ on the r.h.s. has a finite renormalization:

$$
Z_{V}\left(g_{0}^{2}\right) \neq 1
$$

Being finite, $Z_{V}$ can only depend on the coupling $g_{0}^{2}$. Note that eq. (44) (and any other vector WI) can now be expressed in terms of the non-conserved local current; we simply substitute $\hat{\Lambda}_{V}$ by $Z_{V} \Lambda_{V}$. This is the basis of the non-perturbative WI calculation of $Z_{V}$, detailed in sect. 5 .

One can also construct useful WI's based on hadronic correlation functions. For definitiveness, we consider the case in which the operator $O\left(x_{1}, \ldots, x_{n}\right)$ of eq. (40) is

\footnotetext{
${ }^{5}$ The absence of logarithms in such mixing has been explicitly demonstrated in ref. [50] for the axial current at all orders in PT; the situation is analogous for the vector current.
} 
$O\left(x_{1}, x_{2}\right)=P^{12}\left(x_{1}\right) P^{31}\left(x_{2}\right)$, where $P^{12}=\bar{\psi}_{1} \gamma_{5} \psi_{2}$. With $x \neq x_{1}, x_{2}$, so that contact terms do not contribute, eq. (40) becomes

$$
\sum_{\mu} \nabla_{\mu}^{x}\left\langle P^{12}\left(x_{1}\right) \widetilde{V}_{\mu}^{23}(x) P^{31}\left(x_{2}\right)\right\rangle=\left(m_{2}-m_{3}\right)\left\langle P^{12}\left(x_{1}\right) S^{23}(x) P^{31}\left(x_{2}\right)\right\rangle
$$

In analogy to eq. (44), we can use eqs. (10) and (11) in order to express the above identity in terms of the renormalized densities, current and masses. By requiring that the renormalized quantities obey the nominal vector WI, we then find that the product of the quark mass times the scalar density is renormalization group invariant. Thus, we obtain for the RC of the scalar density $S(x)$ :

$$
Z_{S}=Z_{m}^{-1}
$$

\subsection{Axial WI's on the Lattice}

Far less straightforward is the implementation of the axial symmetry with Wilson fermions, because of the presence of the chiral symmetry breaking Wilson term in the action. This topic has been dealt with in refs. [48, 49]. The basic idea is that, by imposing suitable renormalization conditions, PCAC is recovered in the continuum. The axial WI, obtained from eq. (39) with $\alpha_{V}^{a}=0$, is

$$
\begin{aligned}
& i\left\langle\frac{\delta O\left(x_{1}, \ldots, x_{n}\right)}{\delta \alpha_{A}^{a}(x)}\right\rangle=a^{4} \sum_{\mu} \nabla_{x}^{\mu}\left\langle O\left(x_{1}, \ldots, x_{n}\right) \widetilde{A}_{\mu}^{a}(x)\right\rangle- \\
& \quad-a^{4}\left\langle O\left(x_{1}, \ldots, x_{n}\right) \bar{\psi}(x)\left\{\frac{\lambda^{a}}{2}, M_{0}\right\} \gamma_{5} \psi(x)\right\rangle-a^{4}\left\langle O\left(x_{1}, \ldots, x_{n}\right) X^{a}(x)\right\rangle
\end{aligned}
$$

where $\widetilde{A}_{\mu}^{a}(x)$ is a bilinear point-split axial current given by

$$
\begin{aligned}
\widetilde{A}_{\mu}^{a}(x)= & \frac{1}{2}\left[\bar{\psi}(x)\left(\gamma_{\mu} \gamma_{5}\right) U_{\mu}(x) \frac{\lambda^{a}}{2} \psi(x+\mu)+\right. \\
& \left.+\bar{\psi}(x+\mu)\left(\gamma_{\mu} \gamma_{5}\right) U_{\mu}^{\dagger}(x) \frac{\lambda^{a}}{2} \psi(x)\right]
\end{aligned}
$$

The term $X^{a}$ in the above WI is the variation of the Wilson term under axial transformations:

$$
X^{a}(x)=-\frac{1}{2} a\left[\bar{\psi}(x) \frac{\lambda^{a}}{2} \gamma_{5} \overrightarrow{\mathcal{D}}^{2} \psi(x)+\bar{\psi}(x) \overleftarrow{\mathcal{D}}^{2} \frac{\lambda^{a}}{2} \gamma_{5} \psi(x)\right]
$$

where

$$
\begin{aligned}
& a^{2} \overrightarrow{\mathcal{D}}^{2} \psi(x)=\sum_{\mu}\left[U_{\mu}(x) \psi(x+\mu)+U_{\mu}^{\dagger}(x-\mu) \psi(x-\mu)-2 \psi(x)\right] \\
& a^{2} \bar{\psi}(x) \stackrel{\overleftarrow{\mathcal{D}}}{2}=\sum_{\mu}\left[\bar{\psi}(x+\mu) U_{\mu}^{\dagger}(x)+\bar{\psi}(x-\mu) U_{\mu}(x-\mu)-2 \bar{\psi}(x)\right]
\end{aligned}
$$


Unlike the vector current case, $X^{a}$ cannot be cast in the form of a four-divergence.

$X^{a}$ is a dimension- 4 operator which, in the naive continuum limit vanishes, being of the form ( $a \times$ dimension-5 operator). However, it has divergent ME's beyond tree-level. Its mixing with operators of equal and lower dimensions, worked out in ref. [49], can be expressed as follows:

$$
\bar{X}^{a}(x)=X^{a}(x)+\bar{\psi}(x)\left\{\frac{\lambda^{a}}{2}, \bar{M}\right\} \gamma_{5} \psi(x)+\left(Z_{\widetilde{A}}-1\right) \nabla_{x}^{\mu} \widetilde{A}_{\mu}^{a}(x)
$$

where naive dimensional arguments tell us that the mixing constant $Z_{\widetilde{A}}\left(g_{0}^{2}, a m\right)$ is finite, whereas $\bar{M}\left(g_{0}^{2}, M_{0}\right)$ diverges linearly like $a^{-1}$. Logarithmic divergences have been shown to be absent at all orders in PT [50]. The mixing constants $Z_{\widetilde{A}}\left(g_{0}^{2}, a m\right)$ and $\bar{M}\left(g_{0}^{2}, M_{0}\right)$, and therefore $\bar{X}^{a}(x)$, are defined by requiring that the renormalized axial current satisfies the nominal (continuum) axial WI. This amounts to a renormalization condition which ensures that the renormalized theory has the proper chiral symmetry. For example, with $O\left(x_{1}, x_{2}\right)=\psi\left(x_{1}\right) \bar{\psi}\left(x_{2}\right)$, eq. (52) becomes

$$
\begin{aligned}
& \nabla_{x}^{\mu}\left\langle\psi\left(x_{1}\right) Z_{\widetilde{A}} \widetilde{A}_{\mu}^{a}(x) \bar{\psi}\left(x_{2}\right)\right\rangle= \\
& \quad=\left\langle\psi\left(x_{1}\right) \bar{\psi}(x)\left\{\frac{\lambda^{a}}{2},\left(M_{0}-\bar{M}\right)\right\} \gamma_{5} \psi(x) \bar{\psi}\left(x_{2}\right)\right\rangle+\left\langle\psi\left(x_{1}\right) \bar{X}^{a}(x) \bar{\psi}\left(x_{2}\right)\right\rangle- \\
& \quad-\delta\left(x-x_{2}\right)\left\langle\psi\left(x_{1}\right) \bar{\psi}\left(x_{2}\right) \gamma_{5}\right\rangle \frac{\lambda^{a}}{2}-\delta\left(x-x_{1}\right) \frac{\lambda^{a}}{2}\left\langle\gamma_{5} \psi\left(x_{1}\right) \bar{\psi}\left(x_{2}\right)\right\rangle
\end{aligned}
$$

where $X^{a}$ has been replaced by $\bar{X}^{a}$. We now take the continuum limit of the above equation, with the aim of obtaining from it the nominal axial WI for the renormalized quantities. We immediately see that this identity can be recovered from eq. (57) provided that the following renormalization condition is imposed on $\bar{X}^{a}$ :

$$
\lim _{a \rightarrow 0}\left\langle\psi\left(x_{1}\right) \bar{X}^{a}(x) \bar{\psi}\left(x_{2}\right)\right\rangle=0
$$

Moreover, the chiral limit is to be defined as the value $M_{C}$ of $M_{0}$ for which the difference $M_{C}-\bar{M}\left(g_{0}^{2}, M_{C}\right)$ vanishes. Thus, in the chiral limit, eq. (57) becomes

$$
\begin{aligned}
& \nabla_{x}^{\mu}\left\langle\psi\left(x_{1}\right) Z_{\widetilde{A}} \widetilde{A}_{\mu}^{a}(x) \bar{\psi}\left(x_{2}\right)\right\rangle= \\
& \quad-\delta\left(x-x_{2}\right)\left\langle\psi\left(x_{1}\right) \bar{\psi}\left(x_{2}\right) \gamma_{5}\right\rangle \frac{\lambda^{a}}{2}-\delta\left(x-x_{1}\right) \frac{\lambda^{a}}{2}\left\langle\gamma_{5} \psi\left(x_{1}\right) \bar{\psi}\left(x_{2}\right)\right\rangle
\end{aligned}
$$

Expressing the quark fields in terms of their renormalized counterparts, we immediately see that the nominal axial WI is recovered up to vanishing cutoff effects, provided that we interpret the product $\hat{A}_{\mu}^{a}=Z_{\widetilde{A}^{A}} \widetilde{A}_{\mu}^{a}$ as the renormalized axial current. Note that $Z_{\widetilde{A}}\left(g_{0}^{2}, a m\right)$, having been fixed in the continuum limit by eq. (58), does not depend on am (only a regular dependence on am permits a well defined chiral limit). Thus, it is a finite renormalization of the form $Z_{\widetilde{A}}\left(g_{0}^{2}\right)$.

The above arguments rest on the assumption that the chiral point $M_{C}$, defined after eq. (58), is consistent with other definitions (e.g. the vanishing of the quark 
mass in PT or of the pion mass). This has been verified both in 1-loop PT [49] and non-perturbatively (e.g. [16]).

In practice it turns out to be more convenient to work with the lattice local axial current $A_{\mu}^{a}(x)$. We can show, in a fashion analogous to the case of the vector current (c.f. the power counting argument based on eqs. (46) - (48)), that $A_{\mu}^{a}(x)$ has a finite normalization constant $Z_{A}$. Thus we have $\hat{A}_{\mu}^{a}=\lim _{a \rightarrow 0}\left[Z_{\widetilde{A}} \widetilde{A}_{\mu}^{a}\right]=\lim _{a \rightarrow 0}\left[Z_{A} A_{\mu}^{a}\right]$. From now on, the combination $Z_{\widetilde{A}} \widetilde{A}_{\mu}^{a}$ will always be substituted by $Z_{A} A_{\mu}^{a}$ wherever it appears in a WI. Also analogous to the vector current case is the lack of mass dependence of these constants. We therefore have:

$$
Z_{\widetilde{A}}\left(g_{0}^{2}\right), Z_{A}\left(g_{0}^{2}\right) \neq 1
$$

Hadronic axial WI's are also very useful. In eq. (52) we consider the operator $O\left(x_{1}\right)=P^{21}\left(x_{1}\right)=\bar{\psi}_{2}\left(x_{1}\right) \gamma_{5} \psi_{1}\left(x_{1}\right)$ and $x \neq x_{1}$, so that contact terms do not contribute. The resulting WI is

$$
\begin{gathered}
Z_{A} \sum_{\mu} \nabla_{x}^{\mu}\left\langle A_{\mu}^{12}(x) P^{21}\left(x_{1}\right)\right\rangle=\left\langle\bar{X}^{12}(x) P^{21}\left(x_{1}\right)\right\rangle+ \\
+\left[m_{01}+m_{02}-\bar{m}_{1}-\bar{m}_{2}\right]\left\langle P^{12}(x) P^{21}\left(x_{1}\right)\right\rangle
\end{gathered}
$$

where $\bar{m}_{i}\left(g_{0}^{2}, M_{0}\right)$ is the $i^{\text {th }}$ diagonal element of $\bar{M}\left(g_{0}^{2}, M_{0}\right)$. Note that we have replaced $X$ by $\bar{X}$ according to eq. (56). The condition (58) implies that correlation function of the operator $\bar{X}$ vanishes in the continuum limit, except for localized contact terms. Thus, when the points $x_{1}$ and $x$ are kept separate, $\left\langle\bar{X}(x) \hat{P}\left(x_{1}\right)\right\rangle$ vanishes for $a \rightarrow 0$. We now write in eq. (61) the renormalized quark mass as:

$$
m_{R}=\bar{Z}_{m}\left[m_{0}-\bar{m}\left(m_{0}\right)\right]=\bar{Z}_{m}\left[m_{0}-m_{C}-\left.\frac{\partial \bar{m}}{\partial m_{0}}\right|_{m_{C}}\left(m_{0}-m_{C}\right)+\ldots\right]
$$

where we have used the property $\bar{m}\left(m_{C}\right)=m_{C}$. Upon expressing eq. (61) in terms of renormalized quantities and requesting the validity of the axial WI in the continuum limit, we obtain for the $\mathrm{RC} Z_{P}$ of the pseudoscalar density:

$$
Z_{P}=1 / \bar{Z}_{m}
$$

Note that from the WI of eq. (61) we can only determine the combination

$$
2 \rho^{12}=Z_{A}^{-1}\left[m_{01}+m_{02}-\bar{m}_{1}-\bar{m}_{2}\right]=\frac{\sum_{\mu} \nabla_{\mu}^{x}\left\langle A_{\mu}^{12}(x) P^{21}\left(x_{1}\right)\right\rangle}{\left\langle P^{12}(x) P^{21}\left(x_{1}\right)\right\rangle}
$$

but not $Z_{A}$. Again, the above is true up to vanishing cutoff effects.

A very useful WI can be derived taking $O\left(x_{1}, x_{2}\right)=A_{\nu}^{b}\left(x_{1}\right) V_{\rho}^{c}\left(x_{2}\right)$ :

$$
\begin{aligned}
Z_{A} \nabla_{x}^{\mu} & \left\langle A_{\mu}^{a}(x) A_{\nu}^{b}\left(x_{1}\right) V_{\rho}^{c}\left(x_{2}\right)\right\rangle \\
& =\left\langle\bar{\psi}(x)\left\{\frac{1}{2} \lambda^{a},\left(M_{0}-\bar{M}\right)\right\} \gamma_{5} \psi(x) A_{\nu}^{b}\left(x_{1}\right) V_{\rho}^{c}\left(x_{2}\right)\right\rangle+\left\langle\bar{X}^{a}(x) A_{\nu}^{b}\left(x_{1}\right) V_{\rho}^{c}\left(x_{2}\right)\right\rangle+ \\
& +i f^{a b d} \delta\left(x-x_{1}\right)\left\langle V_{\nu}^{d}\left(x_{1}\right) V_{\rho}^{c}\left(x_{2}\right)\right\rangle+i f^{a c d} \delta\left(x-x_{2}\right)\left\langle A_{\nu}^{b}\left(x_{1}\right) A_{\rho}^{d}\left(x_{2}\right)\right\rangle
\end{aligned}
$$


where again use of eq. (56) has been made. By symmetry arguments the contact terms to which $\bar{X}^{a}$ gives rise must have the form

$$
\begin{aligned}
& \left\langle\bar{X}^{a}(x) A_{\nu}^{b}\left(x_{1}\right) V_{\rho}^{c}\left(x_{2}\right)\right\rangle=-i c_{1} f^{a b d} \delta\left(x-x_{1}\right)\left\langle V_{\nu}^{d}\left(x_{1}\right) V_{\rho}^{c}\left(x_{2}\right)\right\rangle+ \\
& \quad+i c_{2} f^{a c d} \delta\left(x-x_{2}\right)\left\langle A_{\nu}^{b}\left(x_{1}\right) A_{\rho}^{d}\left(x_{2}\right)\right\rangle+\ldots
\end{aligned}
$$

where the dots stand for localized Schwinger terms, which will vanish after integration over $x$ (we always keep $x_{1} \neq x_{2}$ ). We now proceed as follows: (I) rewrite eq. (65), using eq. (66) and expressing all bare quantities in terms of the renormalized ones; (II) recall that the $\mathrm{RC} \bar{Z}_{m}$ of $M_{0}-\bar{M}$ is the inverse of the $\mathrm{RC} Z_{P}$ (c.f. eq. (63)); (III) require, as always, that the renormalized quantities obey the nominal axial WI. Thus we obtain

$$
\begin{aligned}
& c_{1}=1-\frac{Z_{V}}{Z_{A}} \\
& c_{2}=\frac{Z_{A}}{Z_{V}}-1
\end{aligned}
$$

The above conditions ensure the recovery of the canonical WI in the continuum limit. This WI is the basis of the non-perturbative calculation of $Z_{A}$ in the fashion of refs. 11, [17, where eqs. (64) - (67) are combined into

$$
\begin{aligned}
& \left\langle\left[\nabla_{x}^{\mu} A_{\mu}^{a}(x)-\bar{\psi}(x)\left\{\frac{\lambda^{a}}{2}, \rho\right\} \gamma_{5} \psi(x)\right] A_{\nu}^{b}\left(x_{1}\right) V_{\rho}^{c}\left(x_{2}\right)\right\rangle= \\
& +i f^{a b d} \delta\left(x-x_{1}\right)\left\langle V_{\nu}^{d}\left(x_{1}\right) V_{\rho}^{c}\left(x_{2}\right)\right\rangle \frac{Z_{V}}{Z_{A}^{2}}+i f^{a c d} \delta\left(x-x_{2}\right)\left\langle A_{\nu}^{b}\left(x_{1}\right) A_{\rho}^{d}\left(x_{2}\right)\right\rangle \frac{1}{Z_{V}}
\end{aligned}
$$

with $\rho=Z_{A}^{-1}\left(M_{0}-\bar{M}\right)$ a matrix generalization of eq. (64). Performing the integration over $x$, and integrating over $\vec{x}_{1}$ (recall that $x_{1} \neq x_{2}$ is necessary in order to eliminate Schwinger terms) we arrive at:

$$
\begin{aligned}
& \int d x \int d \vec{x}_{1}\left\langle\bar{\psi}(x)\left\{\frac{1}{2} \lambda^{a}, \rho\right\} \gamma_{5} \psi(x) A_{\nu}^{b}\left(x_{1}\right) V_{\rho}^{c}\left(x_{2}\right)\right\rangle= \\
& -i \frac{Z_{V}}{Z_{A}^{2}} f^{a b d} \int d \vec{x}_{1}\left\langle V_{\nu}^{d}\left(x_{1}\right) V_{\rho}^{c}\left(x_{2}\right)\right\rangle-i \frac{1}{Z_{V}} f^{a c d} \int d \vec{x}_{1}\left\langle A_{\nu}^{b}\left(x_{1}\right) A_{\rho}^{d}\left(x_{2}\right)\right\rangle
\end{aligned}
$$

Note that this equation is only valid away from the chiral limit, where the integral over $x$ of the total divergence $\nabla_{x}^{\mu} A_{\mu}^{a}(x)$ vanishes. At zero quark mass, the term containing the total divergence contributes because of the presence of massless Goldstone bosons.

The same arguments may be repeated for the operator $O\left(x_{1}, x_{2}\right)=S^{g}\left(x_{1}\right) P^{h}\left(x_{2}\right)$. By imposing that the renormalized operators $\hat{S}^{f}\left(x_{1}\right)$ and $\hat{P}^{f}\left(x_{2}\right)$ satisfy the (integrated) nominal Ward identity, we arrive at

$$
\begin{aligned}
& \int d^{4} x \int d^{3} \vec{y}\left\langle\bar{\psi}(x)\left\{\frac{1}{2} \lambda^{f}, \rho\right\} \psi(x) S^{g}\left(x_{1}\right) P^{h}\left(x_{2}\right)\right\rangle= \\
& \frac{Z_{P}}{Z_{A} Z_{S}} d^{f g l} \int d^{3} \vec{x}_{1}\left\langle P^{l}\left(x_{1}\right) P^{h}\left(x_{2}\right)\right\rangle+\frac{Z_{S}}{Z_{A} Z_{P}} d^{f h l} \int d^{3} \vec{x}_{1}\left\langle S^{g}\left(x_{1}\right) S^{l}\left(x_{2}\right)\right\rangle
\end{aligned}
$$


where the $d$ 's are defined in eq. (田). The WI of eq. (70) is valid if $f \neq g$ and $f \neq h$, otherwise there are additional terms containing flavour-singlet currents on the right hand side. Analogous arguments to the ones used previously, show that the ratio $Z_{S} / Z_{P}$, being obtained from a WI, is a finite function of $g_{0}^{2}$. Thus, the densities $S$ and $P$ have the same anomalous dimension.

Another way of obtaining the ratio $Z_{P} / Z_{S}$ is found by combining eqs. (10), (51), (62) and (63):

$$
\frac{Z_{P}}{Z_{S}}=\frac{m_{0}-\bar{m}\left(m_{0}\right)}{m_{0}-m_{C}}=1-\left.\frac{\partial \bar{m}\left(g_{0}^{2}, m_{0}\right)}{\partial m_{0}}\right|_{m_{0}=m_{C}}+\ldots
$$

where $m_{0}-\bar{m}=2 \rho Z_{A}$.

In this section we have reviewed the lattice vector and axial WI's, emphasizing that they explicitly determine finite RC's such as $Z_{V}, Z_{A}$ and the ratio $Z_{S} / Z_{P}$. We have repeatedly stressed that these results are strictly true up to vanishing $\mathcal{O}(a)$ terms. In the next section we will use the above WI's in order to determine non-perturbatively the RC's. A crucial observation is that these computations are performed at fixed finite cutoff $a$. In this case, the continuum limit is never taken, and an apparent "dependence" of $Z_{O}$ on $a$ is observed. This "dependence" is a discretization (systematic) error, which will eventually drop out in the continuum limit. Minimizing this error is the focal point of the Clover action improvement, discussed in subsection 5.2.

\section{Evaluation of RC's from WI's}

The most accurate method for a non-perturbative determination of RC's is based on chiral WI's. Only finite RC's can be extracted from them, such as $Z_{V}, Z_{A}$ and the ratio $Z_{S} / Z_{P}$. We will use the general results of the previous section in order to show how they can be computed non-perturbatively.

These RC's are determined by requiring that bare correlation functions, calculated numerically on the lattice at finite UV cutoff $a^{-1}$, when renormalized, obey the WI's of the continuum theory. This requirement, which we have justified in the previous section in the $a \rightarrow 0$ limit, should be also valid at very small but finite lattice spacing, within a given accuracy. At fixed lattice spacing, correlation functions can be computed numerically, providing us with an intrinsically non-perturbative estimate of each term of a WI. These WI's can then be solved for the several RC's. Clearly, this method is free from the errors which affect the perturbative estimates of the RC's (i.e. higher orders

in $g_{0}^{2}$ ). It suffers however, from finite cutoff effects. We shall address this question in detail in the rest of this paper.

\subsection{Wilson WI Estimates of Renormalization Constants}

First we consider the WI computation of the RC of the vector current, from suitable ratios of correlation functions. We shall use the following symmetrized form of the 
conserved current:

$$
V_{\mu}^{a C}=\frac{1}{2}\left[\widetilde{V}_{\mu}^{a}(x)+\widetilde{V}_{\mu}^{a}(x-\mu)\right]
$$

Being just a symmetrization of $\widetilde{V}_{\mu}^{a}$, it has the same $\mathrm{RC}, Z_{V^{C}}=1$. From the discussion of the vector WI's between hadronic correlation functions, we conclude that $Z_{V}$ can be derived from ratios of $n$-point correlation functions; e.g.

$$
\begin{aligned}
R_{\rho}(\vec{q}) & =\frac{\int d \vec{x} e^{i \vec{q} \vec{x}}\left\langle V_{k}^{C}(x) M_{\rho}^{k \dagger}(0)\right\rangle}{\int d \vec{x} e^{i \vec{q} \vec{x}}\left\langle V_{k}(x) M_{\rho}^{k \dagger}(0)\right\rangle} \rightarrow \frac{\sum_{r} \epsilon_{r}^{k}(\vec{q})\left\langle 0\left|V_{k}^{C}(0)\right| \rho_{r}(\vec{q})\right\rangle}{\sum_{r} \epsilon_{r}^{k}(\vec{q})\left\langle 0\left|V_{k}(0)\right| \rho_{r}(\vec{q})\right\rangle}+\ldots \\
R_{0}(\vec{q}) & =\frac{\int d \vec{x} \int d \vec{y} e^{i \vec{q} \vec{x}}\left\langle P_{K}(0) V_{0}^{C}(x) P_{D}^{\dagger}(y)\right\rangle}{\int d \vec{x} \int d \vec{y} e^{i \vec{q} \vec{x}}\left\langle P_{K}(0) V_{0}(x) P_{D}^{\dagger}(y)\right\rangle} \rightarrow \frac{\left\langle K(\vec{q})\left|V_{0}^{C}(0)\right| D(\overrightarrow{0})\right\rangle}{\left\langle K(\vec{q})\left|V_{0}(0)\right| D(\overrightarrow{0})\right\rangle}+\ldots \\
R_{k}(\vec{q}) & =\frac{\int d \vec{x} \int d \vec{y} e^{i \vec{q} \vec{x}}\left\langle P_{K}(0) V_{k}^{C}(x) P_{D}^{\dagger}(y)\right\rangle}{\int d \vec{x} \int d \vec{y} e^{i \vec{q} \vec{x}}\left\langle P_{K}(0) V_{k}(x) P_{D}^{\dagger}(y)\right\rangle} \rightarrow \frac{\left\langle K(\vec{q})\left|V_{k}^{C}(0)\right| D(\overrightarrow{0})\right\rangle}{\left\langle K(\vec{q})\left|V_{k}(0)\right| D(\overrightarrow{0})\right\rangle}+\ldots \\
R_{k}^{*}(\vec{q})= & \frac{\int d \vec{x} \int d \vec{y} e^{i \vec{q} \vec{x}}\left\langle M_{K^{*}}^{\lambda}(0) V_{k}^{C}(x) P_{D}^{\dagger}(y)\right\rangle}{\int d \vec{x} \int d \vec{y} e^{i \vec{q} \vec{x}}\left\langle M_{K^{*}}^{\lambda}(0) V_{k}(x) P_{D}^{\dagger}(y)\right\rangle} \rightarrow \frac{\sum_{r} \epsilon_{r}^{\lambda}(\vec{q})\left\langle K_{r}^{*}(\vec{q})\left|V_{k}^{C}(0)\right| D(\overrightarrow{0})\right\rangle}{\sum_{r} \epsilon_{r}^{\lambda}(\vec{q})\left\langle K_{r}^{*}(\vec{q})\left|V_{k}(0)\right| D(\overrightarrow{0})\right\rangle}+\ldots
\end{aligned}
$$

where $k=1,2,3$ is a spatial index and $\epsilon_{r}^{\lambda}(\vec{q})(\lambda=0, \ldots, 3)$ the vector meson polarization vectors. For definitiveness, we have specified the quark flavours by considering the correlations characteristic of the $\rho$ decay (2-point functions) and those of the $D \rightarrow K$ and $D \rightarrow K^{*}$ decays (3-point functions). Thus, the operators are defined as: $P_{K}=\bar{u} \gamma^{5} s ; P_{D}=\bar{u} \gamma^{5} c ; M_{\rho}^{k}=\bar{u} \gamma^{k} d ; M_{K^{*}}^{\lambda}=\bar{u} \gamma^{\lambda} s$. The vector currents have a $\bar{u}-d$ flavour structure in $R_{\rho}$ and a $\bar{u}-c$ flavour structure in all other ratios. In the above equation, we also show the asymptotic behaviour of these ratios, obtained at large time separations, up to contributions from higher excited states. All these ratios give estimates of $Z_{V}$ up to terms of $\mathcal{O}(a)$.

The above ratios represent the most straightforward way to obtain $Z_{V}$, but it is by no means the only one. As an example, we present a method, which follows more closely the vector WI's but is analogous to the determination of $Z_{V}$ from the ratio $R_{0}$. It consists in integrating over all 3 -space $\vec{x}$ both sides of eq. (50). Upon integrating, the spatial derivative $\nabla^{k} \widetilde{V}_{k}$ vanishes (either because fields die-off at infinity or due to periodic boundary conditions of a finite lattice). Then we write the WI in terms of $V_{0}^{C}$. The result is

$$
\int d \vec{x}\left\langle P^{12}\left(x_{1}\right) \nabla_{x}^{0} V_{0}^{C}(x) P^{31}\left(x_{2}\right)\right\rangle=\frac{1}{2}\left(m_{2}-m_{3}\right) \int d \vec{x}\left\langle P^{12}\left(x_{1}\right)\left[S^{23}(x)+S^{23}(x-\hat{0})\right] P^{31}\left(x_{2}\right)\right\rangle
$$

where, upon passing from $\widetilde{V}_{0}$ to $V_{0}^{C}$, we end up with the sum of two adjacent scalar densities on the r.h.s. Finally, we substitute $Z_{V} V_{0}$ for $V_{0}^{C}$ in the above and solve for $Z_{V}$. The implementation of eqs. (73) for the computation of $Z_{V}$ will be referred to as the "Ratio" determination. When WI's are directly used instead, we will call it the WI determination. Both are non-perturbative methods, equivalent in the $a \rightarrow 0$ limit.

For the WI determination of $Z_{A}$ we start from eq. (69). For definitiveness, we will consider 3 quark flavours, which may or may not be degenerate in mass. It is necessary 
to keep track of the flavour content of each operator, e.g. $O_{\Gamma}^{12}(x)=\bar{\psi}_{1}(x) \Gamma \psi_{2}(x)$. The RC's do not depend on flavour. We shall however leave flavour indices on them in order to remind us the origin of possible discretization errors in their determination (e.g. $\mathcal{O}\left(a m_{1}, a m_{2}\right)$ for $Z^{12}$ ). With this flavour content, setting $x_{2}=0$, eq. (69) becomes

$$
\begin{aligned}
& 2 \rho^{12} \int d x \int d \vec{x}_{1}\left\langle P^{12}(x) A_{\nu}^{31}\left(x_{1}\right) V_{\rho}^{23}(0)\right\rangle= \\
& \quad=\frac{Z_{V}^{23}}{Z_{A}^{13} Z_{A}^{12}} \int d \vec{x}_{1}\left\langle V_{\nu}^{32}\left(x_{1}\right) V_{\rho}^{23}(0)\right\rangle-\frac{Z_{A}^{13}}{Z_{A}^{12} Z_{V}^{23}} \int d \vec{x}_{1}\left\langle A_{\nu}^{31}\left(x_{1}\right) A_{\rho}^{13}(0)\right\rangle
\end{aligned}
$$

This WI can be useful in several ways. To simplify matters, in practice we will consider the case of flavours 2 and 3 degenerate in mass. This means that the pre-factors of the two integrals of the r.h.s. of eq. (75) simplify to $Z_{V}^{22} /\left(Z_{A}^{12}\right)^{2}$ and $1 / Z_{V}^{22}$. Thus, by solving the above WI at different times $t_{1}$, we obtain estimates of $Z_{V}^{22}$ and $Z_{A}^{12}$. Alternatively, $Z_{V}$ can be calculated from, say, one of the ratios of eq. (73) and used in eq. (75), from which $Z_{A}$ can be computed. Moreover, with $\nu=\rho=0$, the integrated correlation $\int d \vec{x}_{1}\left\langle V_{0}\left(x_{1}\right) V_{0}(0)\right\rangle$ vanishes, since it is proportional to the charge of the vacuum. This means that the remaining WI

$$
2 \rho^{12} \int d x \int d \vec{x}_{1}\left\langle P^{12}(x) A_{0}^{21}\left(x_{1}\right) V_{0}^{22}(0)\right\rangle=-\frac{1}{Z_{V}^{22}} \int d \vec{x}_{1}\left\langle A_{0}^{21}\left(x_{1}\right) A_{0}^{12}(0)\right\rangle
$$

can be used for yet another determination of $Z_{V}$. Besides $Z_{A}$ and $Z_{V}$, the finite ratio $Z_{S} / Z_{P}$ can also be obtained from the WI of eq. (70), by a method analogous to the one used for $Z_{A}$; eq. (75).

So far only WI's of hadronic correlation functions have been examined. Quark correlation functions such as $\left\langle\psi(x) V_{\mu}(0) \bar{\psi}(y)\right\rangle$ and $\left\langle\psi(x) A_{\mu}(0) \bar{\psi}(y)\right\rangle$ can also be used in principle with WI's such as eqs. (42) and (57). However, in numerical simulations the ratios of hadronic correlation functions turn out to be more stable than those of quark correlation functions. For the latter case, we must work in a fixed gauge and the Gribov ambiguity causes increased statistical fluctuations. Thus, the determination of $Z_{V}$ and $Z_{A}$ from quark state correlation functions is expected to be noisier. This has been explicitly verified for $Z_{A}$ in refs. [17] and [19].

\subsection{Clover-Improved WI Estimates of Renormalization Constants}

As already pointed out, the WI determination of the finite RC's has the advantage of being non-perturbative. In principle, all WI's should give the same finite $Z_{O}$ of a given operator $O$, since the RC's are characteristic of the operator they renormalize, but are independent of the ME's from which they are obtained. In practice, however, problems arise from the fact that the RC's, properly defined at vanishing cutoff, when calculated from WI's (or Ratios), are obtained at finite cutoff. At finite lattice spacing, operators mix with higher dimensional (irrelevant) operators. This mixing spoils the 
renormalization properties of the lattice operators, which is only recovered in the limit $a \rightarrow 0$. The contamination from these higher dimensional operators to the numerical calculation of RC's depends on the correlation functions (or ME's) used. Consequently, the result for $Z_{O}$ obtained at finite cutoff will depend on the correlation functions from which it has been obtained and the quark mass at which the simulation was performed. This unwanted dependence signals the presence of systematic errors which are effectively $\mathcal{O}(a)$ in the scaling limit. More specifically, they are $\mathcal{O}\left(a \Lambda_{Q C D}\right), \mathcal{O}(a m), \mathcal{O}(a q)$ and higher, where $m$ and $q$ stand for the masses and spatial momenta that characterize the process under study.

In order to reduce this systematic error, several proposals have been put forward [51], [30], 31], [32]. All are based on the Symanzik improvement programme [52], which consists in adding suitable irrelevant dimension-5 operators to the lattice action. Moreover, $d$-dimensional operators must also be redefined by the addition of $(d+1)$ dimensional improving operators. All proposals cited above achieve the elimination of the $\mathcal{O}(a)$ discretization error (although at different orders in $\mathrm{PT}$ ), and are referred to as $\mathcal{O}(a)$ improvement. The most widely used improved action is the $\mathrm{SW} /$ Clover one [30], defined as follows:

$$
S_{c}=S_{f}-c_{S W}\left(g_{0}^{2}\right) a^{4} \sum_{x, \mu \nu} \frac{a}{4} \bar{\psi}(x) \sigma_{\mu \nu} F_{\mu \nu}(x) \psi(x)
$$

with $F_{\mu \nu}(x)$ the clover-leaf discretization of the field tensor. The improving coefficient $c_{S W}\left(g_{0}^{2}\right)$ can be calculated in PT [53]; more recently a non-perturbative determination has also been performed [32]. In this work we limit ourselves to its tree-level value $c_{S W}=1$. At this order, all $\mathcal{O}\left(a g_{0}^{2 n} \ln ^{n} a\right)$ terms, which are effectively of $\mathcal{O}(a)$ in the scaling limit $\left(g_{0}^{2} \sim 1 / \ln a\right)$, are eliminated from correlation functions. At leading-log level, the improvement of local operators can be expressed as a rotation of the fermion fields [31]. According to this prescription, improved bilinear operators are given by

$$
O_{\Gamma}^{I}(x)=\bar{\psi}^{R}(x) \Gamma \psi^{R}(x)
$$

where the rotated fields are defined through

$$
\begin{aligned}
\psi^{R}(x) & =\left[1-\frac{a}{4}\left(\overrightarrow{\not D}-m_{0}\right)\right] \psi(x) \\
\bar{\psi}^{R}(x) & =\bar{\psi}(x)\left[1+\frac{a}{4}\left(\overleftarrow{\not D}+m_{0}\right)\right]
\end{aligned}
$$

and the symmetric lattice covariant derivatives are:

$$
\begin{aligned}
& a \vec{D}_{\mu} \psi(x)=\frac{1}{2}\left[U_{\mu}(x) \psi(x+\hat{\mu})-U_{\mu}^{\dagger}(x-\hat{\mu}) \psi(x-\hat{\mu})\right] \\
& a \bar{\psi}(x) \overleftarrow{D}_{\mu}=\frac{1}{2}\left[\bar{\psi}(x+\hat{\mu}) U_{\mu}^{\dagger}(x)-\bar{\psi}(x-\hat{\mu}) U_{\mu}(x-\hat{\mu})\right]
\end{aligned}
$$

The improved operators $O^{I}$ differ from the original ones by terms proportional to the cutoff. Consequently, they have different RC's, say $Z_{O^{I}}$. 
For the Clover action, the quark propagator $\mathcal{S}^{I}(x-y)=\langle\psi(x) \bar{\psi}(y)\rangle$ satisfies the equations

$$
\begin{aligned}
& {\left[\overrightarrow{\not D}(x)+m_{0}+a \vec{W}\right] \mathcal{S}^{I}(x-y)=\delta(x-y)} \\
& \mathcal{S}^{I}(x-y)\left[-\overleftarrow{D D}(y)+m_{0}+a \overleftarrow{W}\right]=\delta(x-y)
\end{aligned}
$$

The terms $\vec{W}$ and $\overleftarrow{W}$ of eq. (81) are shorthand notation for the operators arising from the variation with respect to $\psi$ and $\bar{\psi}$ of the Wilson term of the fermionic action, including the Clover term given by eq. (77). The tree-level $\mathcal{O}(a)$ improved quark propagator is $\left\langle\psi^{R}(x) \bar{\psi}^{R}(y)\right\rangle$. Using eqs. (79) and (81), we can write it in terms of $\mathcal{S}^{I}(x-y)$ :

$$
\left\langle\psi^{R}(x) \bar{\psi}^{R}(y)\right\rangle=\left[1-\frac{a}{2} \overrightarrow{\not D}(x)\right] \mathcal{S}^{I}(x-y)\left[1+\frac{a}{2} \overleftarrow{\not D}(y)\right]+\frac{a}{2} \delta(x-y)+\mathcal{O}\left(a^{2}\right)
$$

where $\mathcal{O}\left(a^{2}\right)$ terms proportional to $\vec{W}$ and $\overleftarrow{W}$ have been dropped, as they do not affect $\mathcal{O}(a)$ improvement. In computing correlation functions between external on-shell hadron states, the $\delta$-function never contributes. Therefore these correlation functions can be expressed directly in terms of the effective rotated propagator

$$
\mathcal{S}^{e f f}(x-y)=\left[1-\frac{a}{2} \overrightarrow{\not D}(x)\right] \mathcal{S}^{I}(x-y)\left[1+\frac{a}{2} \overleftarrow{\not D}(y)\right]
$$

This is the Clover propagator we will mostly use in our computations. The reason it contains an $\mathcal{O}\left(a^{2}\right)$ term in its definition is that it is readily computable in numerical simulations [16]. Thus, it has been extensively used in several Clover improved lattice QCD computations of on-shell ME's. However, upon studying the lattice WI's we find cases for which the space-time points of correlation functions are allowed to coincide. The on-shell ME's argument is then invalidated and the complete propagator of eq. (82) must be used, with the $\delta$-function giving rise to contact terms.

More generally, we can add to the improved propagator $\left\langle\psi^{R}(x) \bar{\psi}^{R}(y)\right\rangle$ the two equations of motion (81), multiplied by an arbitrary factor $z$. Up to $\mathcal{O}\left(a^{2}\right)$, we then find

$$
\begin{aligned}
& \left\langle\psi^{R}(x) \bar{\psi}^{R}(y)\right\rangle_{z}= \\
& \quad\left[1-\frac{a}{2}\left(z \vec{D}(x)-(1-z) m_{0}\right)\right] \mathcal{S}^{I}(x-y)\left[1+\frac{a}{2}\left(\overleftarrow{\not D}(y) z+(1-z) m_{0}\right)\right] \\
& \quad+\frac{a}{2}(2 z-1) \delta(x-y)+\mathcal{O}\left(a^{2}\right)
\end{aligned}
$$

In this case, improved on-shell correlation functions can be expressed in terms of traces of the effective propagator

$$
\mathcal{S}_{z}^{e f f}(x-y)=\left[1-\frac{a}{2}\left(z \overrightarrow{\not D}(x)-(1-z) m_{0}\right)\right] \mathcal{S}^{I}(x-y)\left[1+\frac{a}{2}\left(\overleftarrow{\not D}(y) z+(1-z) m_{0}\right)\right]
$$


with $z$ a free parameter, which we have at our disposal for optimization purposes. Clearly, for different choices of $z$, these ME's (and the corresponding improved operators) differ by $\mathcal{O}\left(a^{2}\right)$ terms. This implies that their RC's also vary with $z$. In this work, unless otherwise stated, we shall use operators $O_{\Gamma}^{I}$ which correspond to $z=1$.

Although on-shell correlation functions of local improved operators $O_{\Gamma}^{I}$ are now improved up to $\mathcal{O}\left(g_{0}^{2} a\right)$, the conserved (point-split) current is not improved. An improved conserved current is given by 15

$$
V_{\mu}^{C I}(x)=V_{\mu}^{C}+\frac{1}{2} a \bar{\nabla}_{\rho}\left(\bar{\psi} \sigma_{\rho \mu} \psi\right)
$$

where the extra " $\sigma_{\rho \mu}$ term" has a symmetric lattice derivative $\left(2 a \bar{\nabla}_{\mu} f(x)=f(x+\mu)-\right.$ $f(x-\mu))$.

Having improved both local and conserved operators, we can now use them in the ratios of eq. (73), in order to obtain estimates of $Z_{V^{I}}$ which only suffer from $\mathcal{O}\left(a g_{0}^{2}\right)$ discretization errors. On the other hand, the l.h.s of the WI of eq. (74), even when expressed in terms of $P^{I}$ and $V_{0}^{C I}$, still suffers from $\mathcal{O}(a)$ corrections. This is due to the presence of the asymmetric lattice derivative $\nabla_{x}^{\mu}=\partial_{x}^{\mu}+\mathcal{O}(a)$. In order to improve it, we must express it in terms of the symmetric derivative $\bar{\nabla}_{x}^{\mu}=\partial_{x}^{\mu}+\mathcal{O}\left(a^{2}\right)$ and make use of the fact that the conserved currents $V_{\mu}^{C}$ and $V_{\mu}^{C I}$ have the same divergence $\left(\bar{\nabla}^{\mu} V_{\mu}^{C}=\bar{\nabla}^{\mu} V_{\mu}^{C I}\right)$. Thus we arrive at the following $\mathcal{O}(a)$ improved version of eq. (74):

$$
\begin{aligned}
& \int d \vec{x}\left\langle P^{12}\left(x_{1}\right) \bar{\nabla}_{x}^{0} V_{0}^{C I}(x) P^{31}\left(x_{2}\right)\right\rangle= \\
& \quad=\frac{\left(m_{2}-m_{3}\right)}{4} \int d \vec{x}\left\langle P^{12}\left(x_{1}\right)\left[2 S^{23}(x)+S^{23}(x+\hat{0})+S^{23}(x-\hat{0})\right] P^{31}\left(x_{2}\right)\right\rangle
\end{aligned}
$$

In the above equation we have dropped the $I$ superscript from the density $P$. The factor $1 / 4$ on the r.h.s. comes from $\bar{\nabla}_{x}^{\mu}$. We can now express $V_{0}^{C I}$ as $Z_{V^{I}} V_{0}^{I}$ and solve for $Z_{V^{I}}$. This estimate of the $\mathrm{RC}$ is free of leading $\mathcal{O}(a)$ errors.

We now turn to the WI of eq. (75) which, expressed in terms of improved operators, can be used for the determination of the tree-level $\mathcal{O}(a)$ improved $\mathrm{RC} Z_{A^{I}}$ of the axial current. The subtlety is that the integration $\int d^{4} x$ of the WI requires inclusion of the contact terms arising from the $\delta$-function of the rotated propagator of eq. (84). Thus, for any value of the $z$-parameter, the Clover improved version of eq. (75) becomes

$$
\begin{aligned}
& 2 \rho^{12} \int d x \int d \vec{y}\left\langle P^{12}(x) A_{\nu}^{31}(y) V_{\rho}^{23}(0)\right\rangle=\left(\frac{Z_{V^{I}}^{23}}{Z_{A^{I}}^{31} Z_{A^{I}}^{12}}-\rho^{12} a \bar{z}\right) \int d \vec{y}\left\langle V_{\nu}^{32}(y) V_{\rho}^{23}(0)\right\rangle \\
& -\left(\frac{Z_{A^{I}}^{13}}{Z_{A^{I}}^{12} Z_{V^{I}}^{23}}-\rho^{13} a \bar{z}\right) \int d \vec{y}\left\langle A_{\nu}^{31}(y) A_{\rho}^{13}(0)\right\rangle
\end{aligned}
$$

where $\bar{z}=(2 z-1)$. All operators in the above equation, as well as the $2 \rho$ factors, are to be understood as improved quantities. For $2 \rho$ this is true provided that it is computed from eq. (64) with improved operators $A_{\mu}^{I}$ and $P^{I}$ and a symmetrized lattice temporal derivative $\bar{\nabla}^{0}$. 


\subsection{Comparison of the Perturbative and WI Estimates of the Renormalization Constants}

From the discussion so far, it should be clear that it is essential to the reliability of any WI computation, performed at finite cutoff, that any scale with mass dimensions (such as $\Lambda_{Q C D}$, masses $m$ and momenta magnitudes $q$ ) must satisfy the condition

$$
\Lambda_{Q C D}, m, q \ll a^{-1}
$$

In an ideal situation ( $g_{0}^{2}$ and $a$ extremely small) the above condition is satisfied and the estimates of the Z's obtained from different correlation functions with the WI/Ratio method should all agree. They should also agree with the $\mathrm{Z}$ estimates obtained in PT, carried out at sufficiently high order. The argument can now be turned upon its head: if the $Z_{O}$ 's obtained from WI's or ratios of different correlation functions are not in good agreement, this is to be interpreted as a signal that the calculation suffers from large discretization errors. In fact, the "dependence" of $Z_{O}$ from the external states and/or the quark mass is a criterion used to estimate the finite lattice spacing effects which affect the lattice calculations. On the other hand, if the WI/Ratio result for $Z_{O}$ turns out to be reliable, any discrepancies with its PT estimate is to be attributed to the truncation of the perturbative series.

To illustrate the point, we collect some results in table 3, obtained at a typical value of the lattice coupling constant, $\beta=6.0$. For the Wilson case, the presence of large $\mathcal{O}(a)$ effects is highlighted by the $20 \%-30 \%$ variation in the various non-perturbative estimates of $Z_{V}$. This wide spread of values renders problematic their comparison to the perturbative estimates (both SPT and BPT). For $Z_{A}$, the non-perturbative values are compatible within statistical errors. However, these errors are too large to allow any conclusion on the accuracy of the two perturbative determinations.

For Clover fermions, the spread of the different WI estimates is reduced to less than $5 \%$. This is due to the elimination of $\mathcal{O}(a)$ terms guaranteed by the Clover improvement. This result implies that lattice QCD calculations, performed with the Clover action at light quark mass, are significantly less affected by the finiteness of the lattice spacing than their Wilson counterparts. Note that BPT predictions for $Z_{V}$ agree well with the non-perturbative results. However, for the ratio $Z_{S} / Z_{P} \mathrm{BPT}$ is less successful. Moreover, the PT estimate of $Z_{A}$ is less than 1 , whereas the NP value exceeds unity. BPT cannot rectify this discrepancy. Thus, $O\left(g^{4}\right)$ terms are probably important in these cases.

So far we have presented results at $\beta=6.0$. The reason for this choice is that this coupling is typically used by present-day lattice QCD groups for calibration purposes. Most studies of an explorative nature (e.g. RC's from WI's, or from the NP method, with or without Clover improvement) have first been carried out at $\beta=6.0$. Thus a comparison of the RC's obtained from all different methods is possible at this value of the coupling. In subsection 7.1 we also present results for $Z_{V}$ obtained from the ratio method with the Wilson action at $\beta=6.4$. Although the cutoff $a^{-1}$ is considerably increased, we will see that finite cutoff effects remain sizeable. Thus, our qualitative 


\begin{tabular}{|c|c|c|c|}
\hline & Method & Wilson & Clover \\
\hline \multirow[t]{6}{*}{$Z_{V}$} & $\overline{\overline{\text { SPT }}}$ & 0.83 & (0.90 \\
\hline & MC-PB(ln $\square)$ & 0.63 & 0.80 \\
\hline & Ratio $\left(R_{\rho}\right)$ & $0.57(1)$ 13 & 0.82 [This work] \\
\hline & Ratio $\left(R_{0}\right)$ & $0.73(3) \quad 13$ & 0.82 [This work] \\
\hline & WI (Axial) & $0.79(4)$ 11 & $0.80(2)$ [This work] \\
\hline & NP & $0.75(5)[24$ & $0.84(1)[22$ \\
\hline \multirow[t]{4}{*}{$\overline{Z_{A}}$} & SPT & 0.87 & 0.98 \\
\hline & MC-PB(ln $\square)$ & 0.72 & 0.95 \\
\hline & WI (Axial) & $0.85(7)$ 11 & 1.11(2) [This work] \\
\hline & $\mathrm{NP}$ & $0.80(5)$ 24 & $1.06(8)[22$ \\
\hline \multirow[t]{4}{*}{$Z_{S} / Z_{P}$} & $\overline{\mathrm{SPT}}$ & 1.10 & 1.20 \\
\hline & MC-PB(ln $\square)$ & 1.34 & 1.54 \\
\hline & WI (Axial) & $\mathrm{N} / \mathrm{A}$ & 1.64(5) [This work] \\
\hline & NP & $\mathrm{N} / \mathrm{A}$ & $1.6(1)[22]$ \\
\hline
\end{tabular}

Table 3: Estimates of the $R C$ 's of the vector current $\left(Z_{V}\right)$, the axial current $\left(Z_{A}\right)$ and the ratio of the scalar and pseudoscalar densities $\left(Z_{S} / Z_{P}\right)$ from various perturbative and nonperturbative methods (see text for labels) at $\beta=6.0$. All results are obtained at a small value of the light quark masses $(a m \simeq 0.07$ ). The corresponding references are shown in square brackets.

conclusions on systematic errors characterizing the Wilson action remain valid at $\beta=$ 6.4. Finally, finite RC's with the Clover action (at light quark mass) have also been computed at $\beta=6.2$ [20].

\section{Lattice - Continuum Normalization of Fields and Currents}

We now examine a proposal due to Lepage, Mackenzie and Kronfeld [36] -38] for the removal of $\mathcal{O}(\mathrm{am})$ systematic errors which are relevant in lattice calculations with the Wilson action at large quark masses. After a critical review of their recipe, we extend it to the Clover case. For both the Wilson and Clover cases, we apply it to the WI computation of $Z_{V}$ and $Z_{A}$ and discuss its merits and shortcomings.

First we set up our notation. So far we have worked with the lattice action $S=$ $S_{f}+S_{g}$ of eqs. (8) and (9), which depends on lattice fields and bare parameters; i.e. it has the form $S\left[\bar{\psi}, \psi, U_{\mu}, m_{0}, g_{0}^{2}, a\right]$. In numerical simulations, it is convenient to work with dimensionless fermion fields and the hopping parameter instead of the quark mass; i.e. the action used in Monte Carlo simulations

$$
S^{L A T}=-\sum_{x, \mu} K\left[\bar{\chi}(x)\left(1-\gamma_{\mu}\right) U_{\mu}(x) \chi(x+\mu)+\bar{\chi}(x+\mu)\left(1+\gamma_{\mu}\right) U_{\mu}^{\dagger}(x) \chi(x)\right]
$$




$$
+\sum_{x} \bar{\chi}(x) \chi(x)+S_{g}
$$

has the form $S^{L A T}\left[\bar{\chi}, \chi, U_{\mu}, K, g_{0}^{2}\right]$, where $K$ and $\chi$ are defined through

$$
\chi=\sqrt{\frac{a^{3}}{2 K}} \psi \quad, \quad \frac{1}{2 K}-4=a m_{0}
$$

Note that the lattice spacing $a$ has been scaled away in $S^{L A T}$. All the equations appearing in the previous sections can now be trivially rewritten in terms of $\bar{\chi}$ and $\chi$ fields and masses in lattice units $m_{0} a$ (or $K$ ); the dependence on $a$ drops out and the equations express relations between dimensionless quantities. From now on, all lattice quantities (such as quark propagators, operators and their correlation functions) are to be understood as defined in terms of the $\bar{\chi}$ and $\chi$ fields; i.e. they are (dimensionless) quantities expressed in lattice units.

In order to connect lattice fields to continuum (bare) ones, we must expand the lattice action for $a \rightarrow 0$ at fixed $g_{0}$ and $K$. This action then reduces to the continuum QCD action, $S^{Q C D}$, provided we relate the continuum field $A_{\mu}$ to its lattice counterpart $U_{\mu}$ in the following way:

$$
U_{\mu}=\exp \left(\operatorname{iag}_{0} A_{\mu}\right)
$$

The standard prescription for the transcription of any continuum quantity on the lattice follows from eqs. (91) and (92). For example, in the naive continuum limit, the dimensionless lattice quark propagator $\mathcal{S}(x-y)=\langle\chi(x) \bar{\chi}(x)\rangle$ becomes its QCD counterpart $\mathcal{S}^{Q C D}(x-y)=\langle\psi(x) \bar{\psi}(x)\rangle$ when normalized as follows:

$$
a^{-3} 2 K \mathcal{S}(x-y) \rightarrow \mathcal{S}^{Q C D}(x-y)
$$

Clearly, such transcriptions are not unique. Modifications of the lattice quantity by terms which are $\mathcal{O}(a)$ give equally acceptable transcriptions. For example, both the lattice local vector current $V_{\mu}(x)=\bar{\chi}_{1}(x) \gamma_{\mu} \chi_{2}(x)$ and the corresponding point-split conserved current $V_{\mu}^{C}(x)$ are good transcriptions of the vector current obtained from $S^{Q C D}$ :

$$
\begin{aligned}
& a^{-3} \sqrt{2 K_{1} 2 K_{2}} V_{\mu}(x) \rightarrow V_{\mu}^{Q C D}(x) \\
& a^{-3} \sqrt{2 K_{1} 2 K_{2}} V_{\mu}^{C}(x) \rightarrow V_{\mu}^{Q C D}(x)
\end{aligned}
$$

Note that these relations are just transcriptions of bare QCD quantities in terms of quantities derived from $S^{L A T}$. They are not relationships between lattice and renormalized quantities, which must involve the presence of RC's; e.g. $\hat{V}_{\mu}=Z_{V} V_{\mu}$. In our notation, the QCD superscript and the "hat" distinguish the two cases.

\subsection{KLM Factors for the Wilson Action}

The proposal by Lepage, Mackenzie and Kronfeld (KLM) [36 -38] is a refinement of the above matching. Their aim is to account for large $\mathcal{O}(\mathrm{am})$ corrections in the 
limit of small $a q$ ( $q$ stands for the magnitude of any relevant spatial momentum). In practice their recipe consists in calculating the matching factor between lattice correlation functions and their continuum counterpart in the free theory, for finite lattice spacing and vanishing spatial momentum. The claim is that this free theory correction should account for most of the $\mathcal{O}(\mathrm{am})$ systematic error also in the interacting case. For example, in the free theory, the matching between the continuum and lattice quark propagators in $(t, \vec{p})$-space at $\vec{p}=\overrightarrow{0}$ is

$$
\mathcal{S}^{Q C D}(t, \vec{p}=\overrightarrow{0})=a^{-3} 2 K\left(1+a m_{0}\right) \mathcal{S}(t, \vec{p}=\overrightarrow{0})
$$

This implies for the lattice fermion field the matching relation

$$
\psi=F_{\psi}\left(a m_{0}\right)^{1 / 2} \sqrt{\frac{2 K}{a^{3}}} \chi
$$

where the KLM wave function normalization factor is

$$
F_{\psi}\left(a m_{0}\right)=1+a m_{0}
$$

This factor tends to 1 in the limit $a m_{0} \rightarrow 0$ and the standard matching of eq. (91) is recovered. We stress that this is a free theory matching which is due to the granularity of the space-time. It is not a renormalization.

A similar procedure applies to all correlation functions. For example consider the correlation function of the lattice local current between external quark states at zero spatial momentum,

$$
L_{\mu}\left(t_{x}, t_{y}\right)=\sum_{\vec{x}, \vec{y}}\left\langle\chi_{1}(y) V_{\mu}(x) \bar{\chi}_{2}(0)\right\rangle
$$

It can easily be seen that, in the free theory and at finite cutoff, it is related to the continuum correlation function by

$$
L_{\mu}^{Q C D}\left(t_{x}, t_{y}\right)=a^{-6} 2 K_{1} 2 K_{2} F_{\psi}\left(a m_{01}\right) F_{\psi}\left(a m_{02}\right) L_{\mu}\left(t_{x}, t_{y}\right)
$$

where $a m_{01}$ and $a m_{02}$ are the bare quark masses of the two distinct flavours of the quark fields. Thus, there is no extra KLM normalization, apart from the one related to the quark fields. The KLM factor for the local vector current turns out to be 1 . This is also the case of the local axial current and of any other local bilinear operator. The KLM factor for the spatial component of the conserved current $V_{k}^{C}(x)$ is also 1 . On the other hand, as pointed out in [54], the temporal component of the conserved current $V_{0}^{C}(x)$ requires an extra KLM factor

$$
F_{V_{0}^{C}}\left(a m_{01}, a m_{02}\right)=\left(1+\frac{a m_{01}+a m_{02}}{2}\right)^{-1}
$$

It arises from the matching of

$$
C_{0}\left(t_{x}, t_{y}\right)=\sum_{\vec{x}, \vec{y}}\left\langle\chi_{1}(y) V_{0}^{C}(x) \bar{\chi}_{2}(0)\right\rangle
$$


to $C_{0}^{Q C D}\left(t_{y}, t_{x}\right)$, which gives:

$$
C_{0}^{Q C D}\left(t_{y}, t_{x}\right)=a^{-6} 2 K_{1} 2 K_{2} F_{\psi}\left(a m_{01}\right) F_{\psi}\left(a m_{02}\right) F_{V_{0}^{C}}\left(a m_{01}, a m_{02}\right) C_{0}\left(t_{y}, t_{x}\right)
$$

It is now trivial to derive the KLM matching factors of hadronic correlation functions. We just have to consider the number of quark fields and the composite operators entering in the correlation function. For example, for the 2-point function of the vector currents, with the $\rho$-meson quantum numbers, we have

$$
\sum_{\vec{x}}\left\langle V_{k}^{C}(x) V_{k}^{\dagger}(0)\right\rangle^{Q C D}=a^{-6} 2 K_{u} 2 K_{d} F_{\psi}\left(a m_{0 u}\right) F_{\psi}\left(a m_{0 d}\right) \sum_{\vec{x}}\left\langle V_{k}^{C}(x) V_{k}^{\dagger}(0)\right\rangle
$$

whereas for the vector 3 -point function of the $D \rightarrow K$ decay we have

$$
\begin{aligned}
\sum_{\vec{x}, \vec{y}}\langle & \left.P_{K}(0) V_{0}^{C}(x) P_{D}^{\dagger}(y)\right\rangle^{Q C D}= \\
& =a^{-6} 2 K_{u} 2 K_{s} 2 K_{c} F_{\psi}\left(a m_{0 u}\right) F_{\psi}\left(a m_{0 s}\right) F_{\psi}\left(a m_{0 c}\right) F_{V_{0}^{C}}\left(a m_{0 s}, a m_{0 c}\right) . \\
& \cdot \sum_{\vec{x}, \vec{y}}\left\langle P_{K}(0) V_{0}^{C}(x) P_{D}^{\dagger}(y)\right\rangle
\end{aligned}
$$

The KLM factors obtained above depend on the bare quark mass of eq. (91). A possible improvement, which could take into account interaction effects, consists in using a more physical definition of the quark mass in the KLM factors. A natural choice is, for instance the subtracted quark mass $m$ which, in terms of the hopping parameter $K$, is given by

$$
a m=\frac{1}{2 K}-\frac{1}{2 K_{C}}
$$

with the critical value $K_{C}$ determined non-perturbatively. Unless otherwise stated, all KLM factors used in our numerical analysis are calculated at the above value of the quark mass (e.g. $F_{\psi}(a m), F_{V_{0}^{C}}(a m)$ etc.). Another proposal has been made in [29], based on the observation that in lattice perturbation theory, a large fraction of renormalization effects comes from tadpole diagrams. The authors of 29] propose to take the bulk of these effects into account by implementing a Mean Field Tadpole Improved (MFTI) prescription. MFT Improvement amounts to the following two substitutions: (1) The link variable in any operator, such as $V_{\mu}^{C}$, is to be substituted by

$$
U_{\mu} \rightarrow \frac{U_{\mu}}{u_{0}}
$$

where $u_{0}$ is any reasonable mean-field estimate of the expectation value of the link. Two standard choices are $u_{0}=\left[\frac{1}{3} \operatorname{Tr} U_{P}\right]^{1 / 4}$ and $u_{0}=1 /\left(8 K_{c}\right)$. In practice, for current values of the coupling constant, the two $u_{0}$ estimates differ by about $10 \%$ (about $2 \%$ for the Clover action). In the results of this work, the latter estimate of $u_{0}$ has been used.

(2) The hopping parameter is obtained by substituting

$$
K \rightarrow \tilde{K}=K u_{0}
$$


This implies, for example, that the MFTI quark mass is obtained by substituting

$$
a m_{0} \rightarrow a \tilde{m}=\frac{1}{2 \tilde{K}}-4=8 K_{C}\left(\frac{1}{2 K}-\frac{1}{2 K_{C}}\right)=8 K_{C} a m
$$

The "standard" MFTI normalization of the quark field is given by the factor $\sqrt{2 \tilde{K}}$; its $\mathrm{KLM} / \mathrm{MFTI}$ correction is obtained through the substitution

$$
\sqrt{2 K} F_{\psi}(a m) \rightarrow \sqrt{2 \tilde{K}} \tilde{F}_{\psi}(a \tilde{m})=\sqrt{2 \tilde{K}(1+a \tilde{m})}
$$

while for the conserved current $V_{0}^{C}$, the KLM/MFTI factor becomes

$$
F_{V_{0}^{C}} \rightarrow \tilde{F}_{V_{0}^{C}}=\frac{1}{u_{0}}\left(1+\frac{a \tilde{m}_{1}+a \tilde{m}_{2}}{2}\right)^{-1}
$$

It must be kept in mind that the $1 / u_{0}$ factor comes from the link $U_{\mu}$ present in the conserved current (i.e. it is a redefinition of the lattice operator due to the redefinition of the gauge field through eq. (106)) whereas the mass dependent factor arises from eq. (108) (i.e. it is a rescaling of the bare mass). This difference in the origin of the two factors will be important when we compute ME's of the conserved currents with KLM/MFTI corrections (see comments after eq. (124)). We also note that the prescription of eq. (108) has also been obtained by a tadpole resummation argument, without recourse to MFTI, in ref. 42.

The KLM factors, having been calculated in the free theory and at zero quark spatial momentum, do not account for, say, $\mathcal{O}\left(g_{0}^{2} a m\right), \mathcal{O}\left(a \Lambda_{Q C D}\right)$ and $\mathcal{O}(a p)$ corrections. The hope is that at large quark mass and small coupling, they account for most of the systematic error due to the finiteness of the UV cutoff. In sect. 7 we will test these expectations.

\subsection{KLM Factors for the Clover Action}

We now calculate the KLM factors for the Clover case. Since the free fermion propagator is the same for both the Wilson and Clover actions, its KLM factor $F_{\psi}$ is the same. However, fermion fields need to be rotated, as for example in eq. (79). Since we usually opt for this specific rotation $(z=1)$, most correlation functions are expressed in terms of the effective lattice propagator $\mathcal{S}^{\operatorname{eff}}(x-y)$, given by eq. (83). The KLM factor for the effective propagator is then given by

$$
\mathcal{S}^{Q C D}(t, \vec{p}=\overrightarrow{0})=a^{-3} \frac{2 K\left(1+a m_{0}\right)}{\left[1+\frac{1}{4}\left(1+a m_{0}-\frac{1}{1+a m_{0}}\right)\right]^{2}} \mathcal{S}^{e f f}(t, \vec{p}=\overrightarrow{0})
$$

where again everything is worked out in the free case. Thus, compared to the Wilson case of eq. (95), there is an extra term, $F_{R}\left(a m_{0}\right)$, arising from the fermion rotations in the improved KLM factor:

$$
F_{\psi}^{I}\left(a m_{0}\right)=F_{\psi}\left(a m_{0}\right) F_{R}\left(a m_{0}\right)=\left(1+a m_{0}\right) F_{R}\left(a m_{0}\right)
$$


with

$$
F_{R}\left(a m_{0}\right)=\left[1+\frac{1}{4}\left(1+a m_{0}-\frac{1}{1+a m_{0}}\right)\right]^{-2}
$$

It can be seen immediately that the overall KLM factor, $F_{\psi}^{I}\left(a m_{0}\right)$, is $1+\mathcal{O}\left(a m_{0}^{2}\right)$, in accordance with $\mathcal{O}(a)$ improvement. From now on, we will directly substitute the bare mass $m_{0}$ by the subtracted one $m$, in the spirit of eq. (105).

Let us consider the quark correlation function of the local improved current $V_{\mu}^{I}=$ $\bar{\chi}_{1}^{R} \gamma_{\mu} \chi_{2}^{R}$ (i.e. the improved analogue of eq. (98)):

$$
L_{\mu}^{I}\left(t_{x}, t_{y}\right)=\sum_{\vec{x}, \vec{y}}\left\langle\chi_{1}^{R}(y) V_{\mu}^{I}(x) \bar{\chi}_{2}^{R}(0)\right\rangle
$$

It is matched to its continuum counterpart through

$$
L_{\mu}^{Q C D}=a^{-6} F_{\psi}^{I}\left(a m_{1}\right) F_{\psi}^{I}\left(a m_{2}\right) L_{\mu}^{I}\left(t_{x}, t_{y}\right)
$$

Thus we see that also in the Clover case the KLM factor for the local vector current, as well as for any other local bilinear operator, is 1.

We now pass to the KLM factors of the conserved improved current of eq. (86). Unlike the improved local current $V_{\mu}^{I}$, fermion fields are not rotated for this current. We first consider the improved quark correlation function of the current's temporal component $V_{0}^{C I}$ :

$$
C_{0}^{I}\left(t_{y}, t_{x}\right)=\sum_{\vec{x}, \vec{y}}\left\langle\chi_{1}^{R}(y) V_{0}^{C I}(x) \bar{\chi}_{2}^{R}(0)\right\rangle
$$

The matching factors for this correlation are easily found to be:

$$
\begin{aligned}
& C_{0}^{Q C D}\left(t_{y}, t_{x}\right)= \\
& a^{-6} 2 K_{1} 2 K_{2} F_{\psi}\left(a m_{1}\right) F_{\psi}\left(a m_{2}\right) F_{R}^{1 / 2}\left(a m_{1}\right) F_{R}^{1 / 2}\left(a m_{2}\right) F_{V_{0}^{C}}\left(a m_{1}, a m_{2}\right) C_{0}^{I}\left(t_{y}, t_{x}\right)
\end{aligned}
$$

where $F_{V_{0}^{C}}$ is given in eq. (100). Note that at $\vec{p}=\overrightarrow{0}$ the $\nabla_{\mu} \bar{\chi} \sigma_{\mu 0} \chi$ term of the current vanishes. The above overall KLM factor in eq. (117) is $1+\mathcal{O}\left(a^{2} m^{2}\right)$, in accordance with improvement.

Finally, we examine the improved correlation $C_{k}^{I}\left(t_{x}, t_{y}\right)$ of the spatial component of the conserved improved current:

$$
C_{k}^{I}\left(t_{y}, t_{x}\right)=\sum_{\vec{x}, \vec{y}}\left\langle\chi_{1}^{R}(y) V_{k}^{C I}(x) \bar{\chi}_{2}^{R}(0)\right\rangle
$$

The standard KLM computation yields the following matching:

$$
\begin{aligned}
& C_{k}^{Q C D}\left(t_{y}, t_{x}\right)= \\
& a^{-6} 2 K_{1} 2 K_{2} F_{\psi}\left(a m_{1}\right) F_{\psi}\left(a m_{2}\right) F_{R}^{1 / 2}\left(a m_{1}\right) F_{R}^{1 / 2}\left(a m_{2}\right) F_{V_{k}^{C I}}\left(a m_{1}, a m_{2}\right) C_{k}^{I}\left(t_{y}, t_{x}\right)
\end{aligned}
$$


where

$$
F_{V_{k}^{C I}}=\left[1-\frac{1-\left(1+a m_{1}\right)^{2}\left(1+a m_{2}\right)^{2}}{4\left(1+a m_{1}\right)\left(1+a m_{2}\right)}\right]^{-1}
$$

Again, being $1+\mathcal{O}\left(a m^{2}\right)$, the overall KLM factor in eq. 119) is consistent with improvement.

We conclude by noting that using the Clover version of the axial WI, in the spirit of KLM, requires a matching factor also for $2 \rho$. From the improved version of eq. (64), with a symmetric lattice temporal derivative $\bar{\nabla}^{0}$, we obtain the KLM matching

$$
2 \rho^{Q C D}=\frac{2\left(a m_{1}+a m_{2}\right)}{\exp \left(a m_{1}+a m_{2}\right)-\exp \left(-a m_{1}-a m_{2}\right)} 2 \rho^{I}
$$

Just like in the Wilson case, $\tilde{m}$ 's and $u_{0}$ 's can be introduced, whenever required by the prescription, in order to account for KLM/MFTI matching factors. Again, the extension of the Clover KLM factors to those characterizing hadronic correlation functions is straightforward.

\section{Non-perturbative Results for the RC's}

In this section we present results for the RC's $Z_{V}$ and $Z_{A}$ and the ratio $Z_{S} / Z_{P}$ obtained, non-perturbatively, from numerical studies of the chiral WI's on the lattice. The RC of the local vector current, $Z_{V}$, has been also computed by taking the ratio of ME's of the conserved to the local vector current between different external states.

The main purpose of this study is to investigate the systematic errors present in these calculations and, in particular, those due to the finiteness of the lattice cutoff. For this purpose, we have considered both the standard Wilson action and the treelevel $\mathcal{O}(a)$-improved Clover action. In order to enhance the finite cutoff effects, we have studied a large range of values of the lattice bare quark masses, which vary from $a m \simeq 0.05$ up to $a m \simeq 1$. As expected, we find that these effects are typically larger with the Wilson action than with the Clover action.

Moreover, we have also tried to correct the finite cutoff effects by following the KLM prescriptions, discussed in the previous section. We find that KLM corrections are typically more effective in the Wilson case, where they are $\mathcal{O}(a m)$ rather than in the Clover case, where they are $\mathcal{O}\left(a^{2} m^{2}\right)$. The analysis of the Clover RC's shows that the bulk of the finite cutoff effects depends linearly on the bare quark mass. This suggests the existence of large $\mathcal{O}\left(g^{2} a m\right)$ terms, which, being a consequence of the quantum loops, cannot be corrected by the tree-level KLM prescriptions.

A significant result is that the finite cutoff effects, even in the Clover case, can be as large as $20-30 \%$, for values of the bare quark masses which are currently used in numerical simulations (e.g. $a m \simeq 0.5$ ). This result strongly supports the necessity of an additional systematic improving strategy, such as [32, 34, 35]. In the following we present our results in detail. 


\subsection{Ratio Determination of $Z_{V}$ with the Wilson Action}

We first present a computation of the $\mathrm{RC}$ of the local vector current, $Z_{V}$, in the case of the standard Wilson action. The data have been obtained by averaging over 20 quenched gauge field configurations, generated at $\beta=6.4$. This value of the lattice coupling constant corresponds to a relatively large value of the lattice cutoff, $a^{-1} \simeq 3.6-3.8$ $\mathrm{GeV}$. The lattice volume is $L^{3} \times T=24^{3} \times 60$. The same set of gauge configurations and quark propagators has already been used in previous phenomenological studies of heavy meson physics on the lattice [55].

$Z_{V}$ has been computed by considering different ratios of two- and three-point correlation functions which reduce, asymptotically in time, to different ME's of the conserved and the local vector currents; cf. eq. (73). For simplicity, these ME's will be denoted in the following as $\left\langle 0\left|V_{k}\right| \rho\right\rangle,\left\langle K\left|V_{\mu}\right| D>\right.$ and $\left\langle K^{*}\left|V_{\mu}\right| D\right\rangle$, regardless of the specific flavour content of the mesons in the external states. In particular, by $D$ we mean a pseudoscalar meson consisting of a "light" and a "heavy" quark, whereas by $K, K^{*}$ and $\rho$ we indicate mesons composed by two light quarks. In all cases, the Wilson parameter of the light quark mass is fixed at $K=0.1485$, the critical value being $K_{C}=0.1506$. Thus this mass corresponds to a bare quark mass $a m \simeq 0.05$. The heavy quark mass varies in the range between $a m \simeq 0.2$ and $a m \simeq 0.6$. In the three point correlation functions, the source of the final $K$ and $K^{*}$ mesons is inserted at the time $t=0$. The $D$ meson is at $t=28$ and the vector current varies in the interval $12 \leq t \leq 16$. The $D$ meson is always considered in its rest frame and the spatial momentum carried by the current is denoted by $\vec{q}$. For simplicity, the momentum transfers $\vec{q}=(0,0,0)$ and $\vec{q}=(1,0,0)$ (expressed in lattice units of $2 \pi / L a)$ will be abbreviated as $q=0$ and $q=1$ respectively.

A first way of obtaining $Z_{V}$ consists in computing the ratio $R_{0}$ of three point correlation functions defined in eq. (73). This ratio has been computed at momentum transfer $q=0$. In the large time limit, it is given by:

$$
R_{0}(q=0)=\frac{<K\left|V_{0}^{C}\right| D>}{<K\left|V_{0}\right| D>}=Z_{V}+\mathcal{O}(a)
$$

According to the KLM claim, the bulk of the $\mathcal{O}(a)$ corrections in this expression should be given by the KLM factor $F_{V_{0}^{C}}\left(a m_{1}, a m_{2}\right)$ of eq. 100) associated with the vertex of the conserved current $V_{0}^{C}$ (the KLM factors $F_{\psi}(a m)$, associated with the external quark fields, cancel in $\left.R_{0}\right)$. Therefore we also define the ratio:

$$
R_{0}^{K L M}=R_{0} \cdot F_{V_{0}^{C}}\left(a m_{1}, a m_{2}\right)=R_{0} \cdot\left(1+\frac{a m_{1}+a m_{2}}{2}\right)^{-1}=Z_{V}+\mathcal{O}(a)
$$

If the KLM prescription works in this case, the $\mathcal{O}(a)$ terms in eq. (123) should be smaller than those in eq. (122). This means that the ratio $R_{0}^{K L M}$ should exhibit a milder dependence on the two quark masses. In addition, we also consider the MFTI version of the ratio $R_{0}$. It is obtained from eq. (123), by substituting the bare quark 


\begin{tabular}{|c|c|c|c|c|c|}
\hline$K_{1}$ & $K_{2}$ & $a m_{\text {eff }}$ & $R_{0}$ & $R_{0}^{K L M}$ & $R_{0}^{M F T T}$ \\
\hline 0.1275 & 0.1275 & 0.602 & 1.278 & 0.798 & 0.741 \\
0.1275 & 0.1325 & 0.528 & 1.207 & 0.790 & 0.738 \\
0.1275 & 0.1375 & 0.459 & 1.141 & 0.782 & 0.735 \\
0.1325 & 0.1325 & 0.454 & 1.136 & 0.782 & 0.735 \\
0.1275 & 0.1425 & 0.395 & 1.081 & 0.775 & 0.732 \\
0.1325 & 0.1375 & 0.385 & 1.070 & 0.773 & 0.731 \\
0.1275 & 0.1485 & 0.324 & 1.017 & 0.768 & 0.731 \\
0.1325 & 0.1425 & 0.321 & 1.010 & 0.764 & 0.728 \\
0.1375 & 0.1375 & 0.316 & 1.005 & 0.763 & 0.728 \\
0.1375 & 0.1425 & 0.253 & 0.945 & 0.754 & 0.725 \\
0.1325 & 0.1485 & 0.250 & 0.946 & 0.756 & 0.727 \\
0.1425 & 0.1425 & 0.189 & 0.885 & 0.744 & 0.721 \\
0.1375 & 0.1485 & 0.182 & 0.880 & 0.745 & 0.722 \\
0.1425 & 0.1485 & 0.118 & 0.820 & 0.733 & 0.718 \\
\hline
\end{tabular}

Table 4: The ratios $R_{0}, R_{0}^{K L M}$ and $R_{0}^{M F T I}$ for the Wilson action and for different values of the quark masses. In the three point correlation functions the light spectator quark has mass $a m \simeq 0.05$. The statistical errors in the table are less than (or equal to) 1 on the last digit.

masses with their MFTI expressions:

$$
R_{0}^{M F T I}=R_{0} \cdot\left(1+\frac{a \tilde{m}_{1}+a \tilde{m}_{2}}{2}\right)^{-1}=Z_{V}+\mathcal{O}(a)
$$

Notice that, with respect to the definition of the KLM/MFTI factor $\tilde{F}_{V_{0}^{C}}$ given in eq. (110), we have omitted in the previous equation a factor $u_{0}$. As pointed out after eq. (110), its presence would imply a redefinition of the conserved current, whereas we are only interested in a comparison of the discretization errors with and without KLM/MFTI corrections. Therefore, by omitting $u_{0}$, we are computing the ratio $R_{0}^{M F T I}$ of the ME's of the same operators which appear in the two ratios $R_{0}$ and $R_{0}^{K L M}$. In this way, all three ratios give estimates of the same $Z_{V}$ and only differ by $\mathcal{O}(a)$ effects.

The values of the three ratios $R_{0}$ are shown in table 4 , for different values of the hopping parameter. They are also plotted in figure 1 as a function of the "effective" quark mass, $a m_{e f f}=\left(a m_{1}+a m_{2}\right) / 2$. We see that $R_{0}$ has a strong dependence on the bare quark masses, varying by approximately $50 \%$ as the mass varies from $a m \simeq 0.2$ to $a m \simeq 0.6$. This dependence is however significantly reduced when we apply the KLM correction. This reduction is somewhat bigger in the MFTI prescription.

In order to discuss these results in a more quantitative way, we fit the three ratios $R_{0}$, as a function of the quark masses, taking into account contributions up to quadratic terms:

$$
R_{0}=A+B\left(a m_{1}+a m_{2}\right)+C\left(a m_{1}+a m_{2}\right)^{2}+D\left(a m_{1}-a m_{2}\right)^{2}
$$




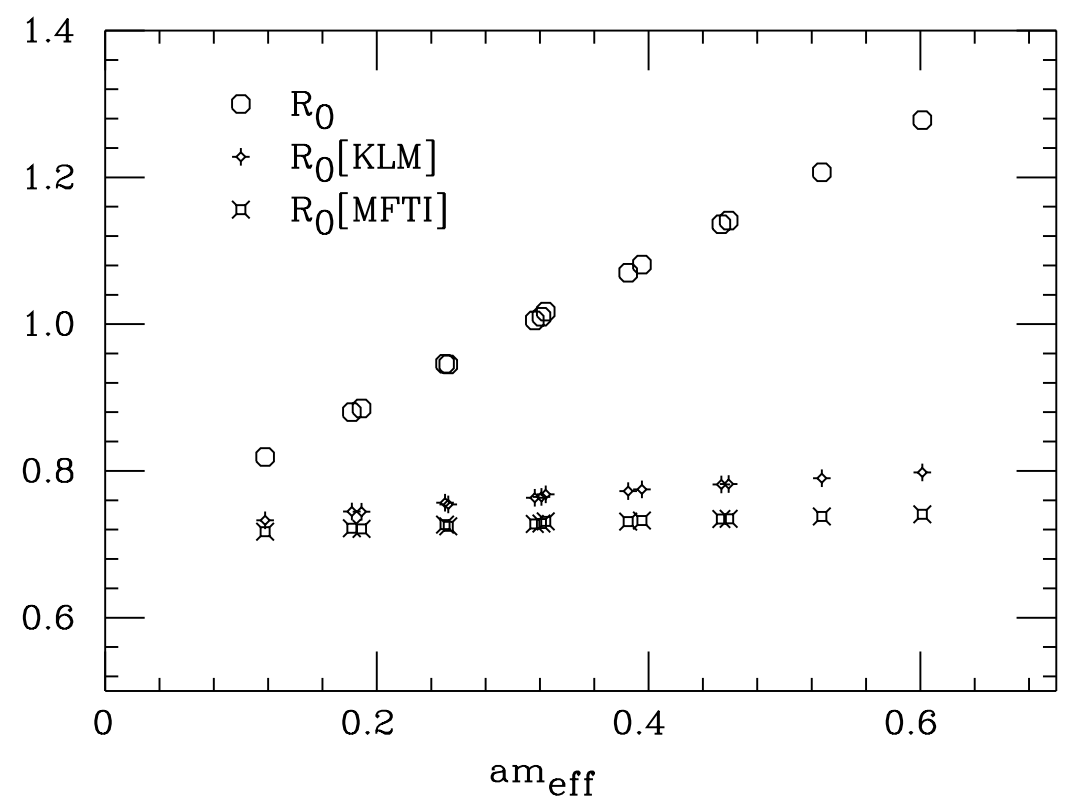

Figure 1: The various ratios $R_{0}$, defined in eqs. (129), (123) and (124) for Wilson fermions, as functions of the effective quark mass.

Since the current vertex is symmetric under interchange of the two quark fields, there is no linear dependence on the mass difference $\left(a m_{1}-a m_{2}\right)$. For all three ratios $R_{0}$, $R_{0}^{K L M}$ and $R_{0}^{M F T I}$ the value of the coefficient $A$ in eq. (125) gives an estimate of $Z_{V}$, with presumably negligible $\mathcal{O}(\mathrm{am})$ systematic errors, which we denote by $Z_{V}^{e f f}$. The result in all three cases is approximately the same and we quote, as an overall estimate:

$$
Z_{V}^{e f f}=0.71(1) \quad, \quad \text { from } R_{0}
$$

The parameter $B$ in eq. (125) gives the size of the leading $\mathcal{O}(\mathrm{am})$ corrections. We find that $B$ decreases from $B \simeq 0.46$ for $R_{0}$, to $B \simeq 0.09$ for $R_{0}^{K L M}$ and to an even smaller value, $B \simeq 0.03$, for $R_{0}^{M F T I}$. The coefficients $C$ and $D$ of the quadratic terms are always of the order $10^{-2}$ or smaller in magnitude. Thus, in the case of the ratio $R_{0}$, the KLM prescription correctly takes into account and removes the bulk of the finite cutoff effects.

Next we compare results for $Z_{V}$ obtained from different ratios of correlation functions, cf. eq. (73). In particular, for different values of the quark masses, we have computed:

$$
\begin{aligned}
& R_{\rho}(q=0)=\frac{\sum_{r} \epsilon_{r}^{k}\left\langle 0\left|V_{k}^{C}(0)\right| \rho_{r}\right\rangle}{\sum_{r} \epsilon_{r}^{k}\left\langle 0\left|V_{k}(0)\right| \rho_{r}\right\rangle}=Z_{V}+\mathcal{O}(a) \\
& R_{k}(q=1)=\frac{\left\langle K\left|V_{k}^{C}(0)\right| D\right\rangle}{\left\langle K\left|V_{k}(0)\right| D\right\rangle}=Z_{V}+\mathcal{O}(a) \\
& R_{k}^{*}(q=1)=\frac{\sum_{r} \epsilon_{r}^{\lambda}\left\langle K_{r}^{*}\left|V_{k}^{C}(0)\right| D\right\rangle}{\sum_{r} \epsilon_{r}^{\lambda}\left\langle K_{r}^{*}\left|V_{k}(0)\right| D\right\rangle}=Z_{V}+\mathcal{O}(a)
\end{aligned}
$$




\begin{tabular}{|c|c|c|c|c|c|}
\hline$K_{1}$ & $K_{2}$ & $a m_{e f f}$ & $R_{\rho}$ & $R_{k}$ & $R_{k}^{*}$ \\
\hline 0.1275 & 0.1275 & 0.602 & $0.622(1)$ & $1.073(41)$ & $0.643(23)$ \\
0.1275 & 0.1325 & 0.528 & $0.628(1)$ & $0.972(37)$ & $0.639(23)$ \\
0.1275 & 0.1375 & 0.459 & $0.633(1)$ & $0.877(34)$ & $0.634(23)$ \\
0.1325 & 0.1325 & 0.454 & $0.632(1)$ & $0.979(38)$ & $0.634(23)$ \\
0.1275 & 0.1425 & 0.395 & $0.638(1)$ & $0.792(30)$ & $0.628(22)$ \\
0.1325 & 0.1375 & 0.385 & $0.637(1)$ & $0.883(34)$ & $0.630(23)$ \\
0.1275 & 0.1485 & 0.324 & $0.643(2)$ & $0.719(20)$ & $0.625(21)$ \\
0.1325 & 0.1425 & 0.321 & $0.642(1)$ & $0.795(30)$ & $0.624(23)$ \\
0.1375 & 0.1375 & 0.316 & $0.641(1)$ & $0.891(34)$ & $0.625(23)$ \\
0.1375 & 0.1425 & 0.253 & $0.645(1)$ & $0.801(30)$ & $0.620(23)$ \\
0.1325 & 0.1485 & 0.250 & $0.646(2)$ & $0.721(20)$ & $0.623(22)$ \\
0.1425 & 0.1425 & 0.189 & $0.649(1)$ & $0.812(30)$ & $0.616(23)$ \\
0.1375 & 0.1485 & 0.182 & $0.649(2)$ & $0.723(19)$ & $0.621(22)$ \\
0.1425 & 0.1485 & 0.118 & $0.653(2)$ & $0.727(19)$ & $0.618(23)$ \\
0.1485 & 0.1485 & 0.047 & $0.658(2)$ & - & - \\
\hline
\end{tabular}

Table 5: The ratios $R_{\rho}, R_{k}$ and $R_{k}^{*}$ for the Wilson action at different values of the vector current quark masses. In the three point correlation functions the light spectator quark has mass $a m \simeq 0.05$.

The KLM corrections to the correlation functions of these ratios always cancel out between numerator and denominator. Thus, according to the KLM expectations, all these ratios should be free of large $\mathcal{O}(a m)$ corrections.

The values of $R_{\rho}, R_{k}$ and $R_{k}^{*}$ are given in table for different values of the vector current quark masses. The same ratios are also plotted in figure 2 as a function of the effective quark mass. In the figure, the ratio $R_{0}^{K L M}$ is also plotted for comparison. We see that $R_{\rho}, R_{k}$ and $R_{k}^{*}$ exhibit quite a different behaviour as a function of the quark masses. When the mass varies from $a m \simeq 0.2$ to $a m \simeq 0.6$, the ratios $R_{\rho}$ and $R_{k}^{*}$ remain almost constant, while the ratio $R_{k}$ is found to be affected by large $\mathcal{O}(a)$ corrections. In particular, by performing a fit as in eq. (125), we find a large contribution coming from the term proportional to $\left(a m_{1}-a m_{2}\right)^{2}$, with a coefficient $D \simeq-0.51$. Compared to its values corresponding to the three previous estimates of $R_{0}, D$ of $R_{k}$ is larger by approximately an order of magnitude. The total finite cutoff effects in the ratio $R_{k}$ can be as large as $50 \%$ for $a m \simeq 0.6$. These effects cannot be corrected by the KLM prescription.

The values of the coefficients $A$ of the fits give an estimate of $Z_{V}$ with presumably negligible $\mathcal{O}(a m)$ effects. We quote:

$$
\begin{array}{lll}
Z_{V}^{e f f}=0.66(1) \quad & , \quad & \text { from } R_{\rho} \\
Z_{V}^{e f f}=0.68(1) & , & \text { from } R_{k} \\
Z_{V}^{e f f}=0.62(1) \quad, & \text { from } R_{k}^{*}
\end{array}
$$



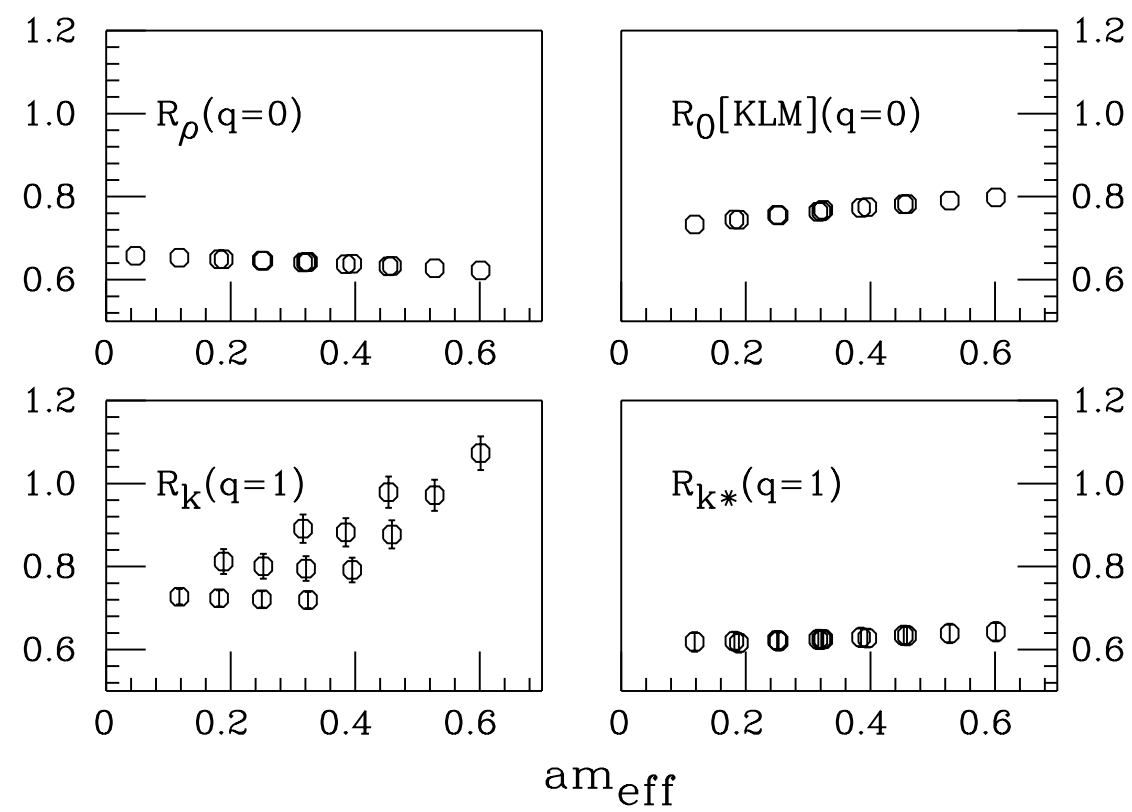

Figure 2: The ratios $R_{\rho}, R_{0}^{K L M}, R_{k}$ and $R_{k}^{*}$, for Wilson fermions, as functions of the effective quark mass.

The differences between these values and the $R_{0}$ estimate of eq. (126) for $Z_{V}$ represent a measure of the $\mathcal{O}(a)$ corrections which remain after the $\mathcal{O}(a m)$ terms have been presumably removed by the fit. These corrections, which are expected to be $\mathcal{O}(a q)$ or $\mathcal{O}\left(a \Lambda_{Q C D}\right)$, leave a systematic uncertainty which is approximately $15 \%$.

\subsection{Ratio Determination of $Z_{V}, Z_{A}$ and $Z_{P} / Z_{S}$ with the Clover Action and Light Quark Masses}

In this subsection we present the results of a calculation of the RC's $Z_{V^{I}}, Z_{A^{I}}$ and the ratio $Z_{P^{I}} / Z_{S^{I}}$ obtained with the tree-level $\mathcal{O}(a)$-improved Clover action, by using the lattice chiral WI's. From now on, the superscript " $I$ " of the $Z_{O^{I}}$ 's will be dropped. We limit ourselves to light values of the bare quark masses. The value of the lattice coupling constant is $\beta=6.0$. The lattice volume is $16^{3} \times 32$. We have generated 18 quenched gauge field configurations. We have worked at three values of the Wilson hopping parameters, namely $K=0.1410,0.1425$ and 0.1432 corresponding to bare quark masses ranging from $a m=0.056$ to $a m=0.111$. The critical value is $K_{C}=0.14554$.

All two- and three-point correlation functions have been computed by considering degenerate quark masses. The results are a replication of those of ref. [17] for three quark masses. The RC of the local axial current $Z_{A}$ and the ratio $Z_{P} / Z_{S}$ have been obtained from gauge invariant chiral WI's, between external hadron states (see eqs. (69) and (70) ). Gauge dependent chiral WI's between external quark states have also been implemented for a calculation of $Z_{A}$ (see eq. (57)). The RC of the local vector current, $Z_{V}$, has been computed from the ratio $R_{\rho}$, with zero spatial momenta. The results of 


\begin{tabular}{|c|c|c|c|c|c|}
\hline$K_{l}$ & $a m_{l}$ & $Z_{V}$ & $Z_{A}$ (g.i.) & $Z_{A}$ (g.d.) & $Z_{P} / Z_{S}$ \\
\hline 0.1410 & 0.111 & $0.833(1)$ & $1.06(5)$ & $1.07(7)$ & $0.61(3)$ \\
0.1425 & 0.073 & $0.824(2)$ & $1.09(3)$ & $1.14(8)$ & $0.60(2)$ \\
0.1432 & 0.056 & $0.819(2)$ & $1.07(7)$ & $1.17(13)$ & $0.60(3)$ \\
\hline
\end{tabular}

Table 6: The $R C^{\prime}$ s $Z_{V}, Z_{A}$ and the ratio $Z_{S} / Z_{P}$ for the Clover action with light quark masses. The labels "g.i." and "g.d" distinguish results obtained from gauge-invariant and gauge-dependent WI's respectively.

this computation are presented in table 6 .

We see that, in the case of $Z_{V}$, the statistical errors are sufficiently small to reveal the presence of finite cutoff effects in the calculation. By performing a fit as in eq. (125) we can obtain the value of $Z_{V}$, with presumably negligible am effects. We quote:

$$
Z_{V}^{e f f}=0.80(1) \quad, \quad \text { from } R_{\rho}
$$

As seen in table 6, the variations of the $\mathrm{RC} Z_{A}$ and the ratio $Z_{S} / Z_{P}$ with the light quark masses are of the size of the statistical errors. Thus, in these cases the presence of finite cutoff effects cannot be determined. An analogous study at $\beta=6.2$ has been performed by the UKQCD collaboration 20].

\subsection{Ratio Determination of $Z_{V}$ with the Clover Action and Heavy Quark Masses}

In order to investigate the size of finite cutoff effects when the tree-level $\mathcal{O}(a)$-improved Clover action is used, we have also computed $Z_{V}$ at several large values of the quark mass. We have generated 30 quenched gauge field configurations at $\beta=6.0$. The lattice volume is $16^{3} \times 48$. Two different values of the light quark hopping parameter have been considered $(K=0.1425$ and $K=0.1432)$ and four different values of the heavy quark mass. The latter correspond to bare quark masses varying from $a m \simeq 0.3$ up to $a m \simeq 0.9$.

We have computed $Z_{V}$ from the ratios $R_{0}$ and $R_{\rho}$ of eq. (73), with quark propagators improved according to eq. (83). All external states and currents are always at zero spatial momentum. In the three-point correlation functions the pseudoscalar $D$ meson is inserted in the middle of the lattice, at $t=24$, so that results from both positive and negative times can be averaged, thus improving the statistics.

In order to investigate the effects of the KLM corrections in the Clover case, we have also considered the corresponding KLM-improved versions of the ratios $R_{0}$ and $R_{\rho}$. According to eqs. (115), (117) and (119), these corrections must be defined as:

$$
R_{0}^{K L M}=\frac{F_{R}\left(a m_{1}\right)^{1 / 2} F_{R}\left(a m_{2}\right)^{1 / 2}}{F_{V_{0}^{C}}\left(a m_{1}, a m_{2}\right)} \cdot R_{0}=Z_{V}+\mathcal{O}\left(g^{2} a, a^{2}\right)
$$




\begin{tabular}{|c|c|c|c|c|c|c|}
\hline$K_{1}$ & $K_{2}$ & $a m_{e f f}$ & $R_{0}$ & $R_{0}^{K L M}$ & $R_{\rho}$ & $R_{\rho}^{K L M}$ \\
\hline 0.1150 & 0.1150 & 0.912 & 0.999 & 0.949 & 1.026 & 1.009 \\
0.1150 & 0.1200 & 0.822 & 0.989 & 0.942 & 1.000 & 0.991 \\
0.1150 & 0.1250 & 0.739 & 0.981 & 0.936 & 0.978 & 0.976 \\
0.1200 & 0.1200 & 0.731 & 0.974 & 0.934 & 0.977 & 0.974 \\
0.1200 & 0.1250 & 0.648 & 0.962 & 0.926 & 0.958 & 0.959 \\
0.1150 & 0.1330 & 0.618 & 0.977 & 0.929 & 0.947 & 0.950 \\
0.1250 & 0.1250 & 0.565 & 0.946 & 0.917 & 0.937 & 0.941 \\
0.1200 & 0.1330 & 0.528 & 0.951 & 0.916 & 0.927 & 0.932 \\
0.1150 & 0.1425 & 0.493 & 0.996 & 0.931 & 0.919 & 0.922 \\
0.1150 & 0.1432 & 0.484 & 0.999 & 0.932 & 0.918 & 0.920 \\
0.1250 & 0.1330 & 0.445 & 0.927 & 0.902 & 0.910 & 0.916 \\
0.1200 & 0.1425 & 0.402 & 0.959 & 0.913 & 0.901 & 0.903 \\
0.1200 & 0.1432 & 0.394 & 0.962 & 0.914 & 0.899 & 0.902 \\
0.1330 & 0.1330 & 0.324 & 0.894 & 0.881 & 0.883 & 0.889 \\
0.1250 & 0.1425 & 0.319 & 0.924 & 0.893 & 0.882 & 0.885 \\
0.1250 & 0.1432 & 0.310 & 0.926 & 0.894 & 0.881 & 0.883 \\
0.1330 & 0.1425 & 0.195 & 0.872 & 0.861 & 0.855 & 0.858 \\
0.1330 & 0.1432 & 0.190 & 0.872 & 0.860 & 0.853 & 0.855 \\
0.1425 & 0.1425 & 0.073 & 0.823 & 0.822 & 0.823 & 0.824 \\
0.1425 & 0.1432 & 0.065 & 0.820 & 0.819 & 0.821 & 0.821 \\
0.1432 & 0.1432 & 0.056 & 0.817 & 0.817 & 0.818 & 0.818 \\
\hline
\end{tabular}

Table 7: The ratios $R_{0}$ and $R_{\rho}$, and their KLM-improved counterparts, for the Clover action at different values of the quark masses. The statistical errors are always less than (or equal to) 1 on the last digit.

$$
R_{\rho}^{K L M}=\frac{F_{R}\left(a m_{1}\right)^{1 / 2} F_{R}\left(a m_{2}\right)^{1 / 2}}{F_{V_{k}^{C I}}\left(a m_{1}, a m_{2}\right)} \cdot R_{\rho}=Z_{V}+\mathcal{O}\left(g^{2} a, a^{2}\right)
$$

where the several KLM factors are given in eqs. (100), (113) and (120). Notice that, in the Clover case, the KLM corrections are always of $\mathcal{O}\left(a^{2}\right)$.

The values of $R_{0}, R_{\rho}$ and their KLM counterparts are given in table 7 for several values of the quark masses. The same ratios are also plotted in figures 3 and 4 , with and without the KLM corrections, as a function of the effective mass $a m_{\text {eff }}=$ $\left(a m_{1}+a m_{2}\right) / 2$. The main feature shown in figure 3 is that both $R_{0}$ and $R_{\rho}$ receive a large contribution from a term which is linear in the quark mass. This term affects the value of the two ratios by approximately $15 \%$ at $a m \simeq 0.5$ and by about $25 \%$ at $a m \simeq 0.9$. Moreover, such a large systematic error cannot be corrected by the KLM factors, the latter being at least quadratic in the quark masses. In fact, a qualitatively similar behaviour is found for the points in figure 1 , where the KLM correction has been implemented.

By looking at figure 3 we also notice that the values of $R_{0}$, obtained with degenerate 


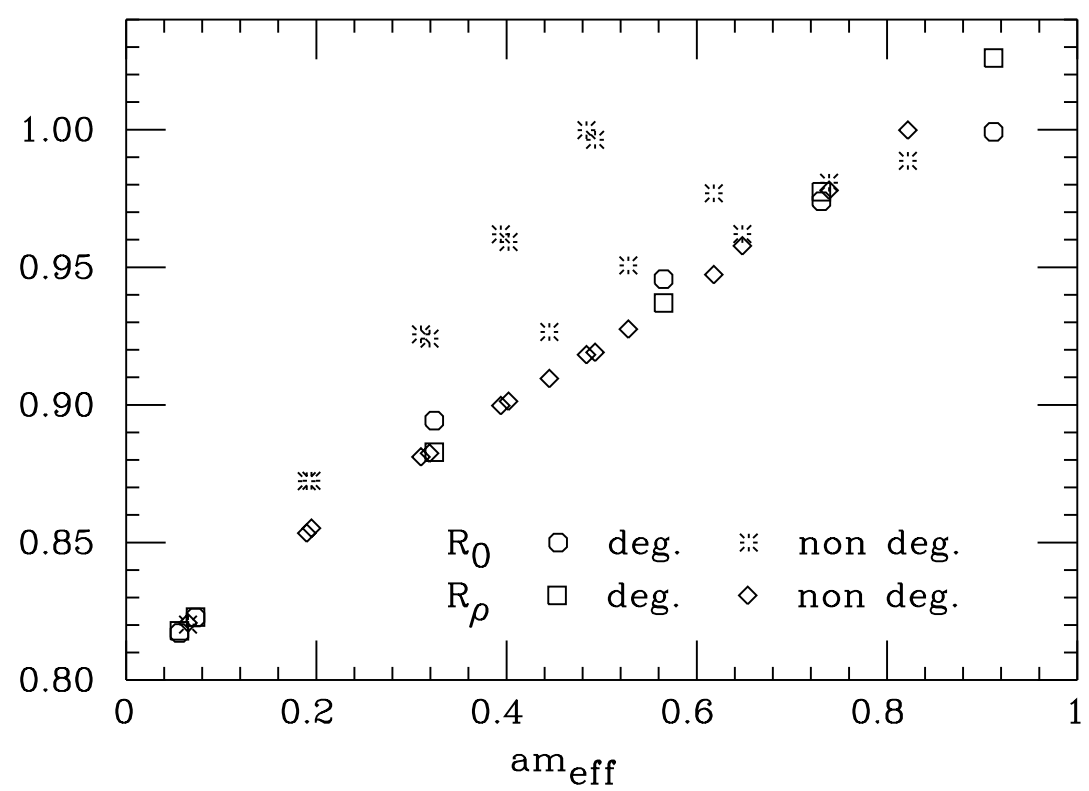

Figure 3: The ratios $R_{0}$ and $R_{\rho}$, for Clover fermions, as a function of the effective quark mass. We distinguish between the cases of degenerate and non-degenerate quarks in the current.

\begin{tabular}{|l|cccc|}
\hline & $A$ & $B$ & $C$ & $D$ \\
\hline$R_{0}$ & 0.80 & 0.19 & -0.05 & 0.09 \\
$R_{0}^{K L M}$ & 0.80 & 0.16 & -0.05 & 0.03 \\
\hline$R_{\rho}$ & 0.81 & 0.12 & -0.0008 & -0.002 \\
$R_{\rho}^{K L M}$ & 0.80 & 0.14 & -0.02 & -0.008 \\
\hline
\end{tabular}

Table 8: Values of the coefficients $A, B, C$ and $D$ obtained from a fit of the ratios $R_{0}, R_{\rho}$ and their KLM-improved counterparts.

quark masses of the vector current, and those obtained by non-degenerate quarks, do not lie on the same curve. This suggests a significant dependence of this ratio on terms quadratic in the mass difference $\left(a m_{1}-a m_{2}\right)^{2}$. In this respect, figure 1 shows that the KLM correction improves the situation, since the difference between degenerate and non-degenerate points is largely reduced. However, the KLM prescription cannot correct large $\mathcal{O}\left(g^{2} a m\right)$ effects, which are evidently present in figure 4 . It would also appear from this figure that such terms are independent of the ME's, since the difference between $R_{0}$ and $R_{\rho}$ settles only after masses of approximately am $\simeq 0.5$.

To make this discussion more quantitative, we fit the ratios $R_{0}, R_{\rho}$ and their KLM counterparts, as in eq. (125). The resulting values of the coefficients $A, B, C$ and $D$ are given in table 8 . The values of the coefficient $A$, in table 8 , represent an estimate of $Z_{V}$ which is presumably free from any $\mathcal{O}(\mathrm{am})$ corrections. We then quote:

$$
Z_{V}^{e f f}=0.80(1) \quad, \quad \text { from } R_{0}, R_{\rho}
$$




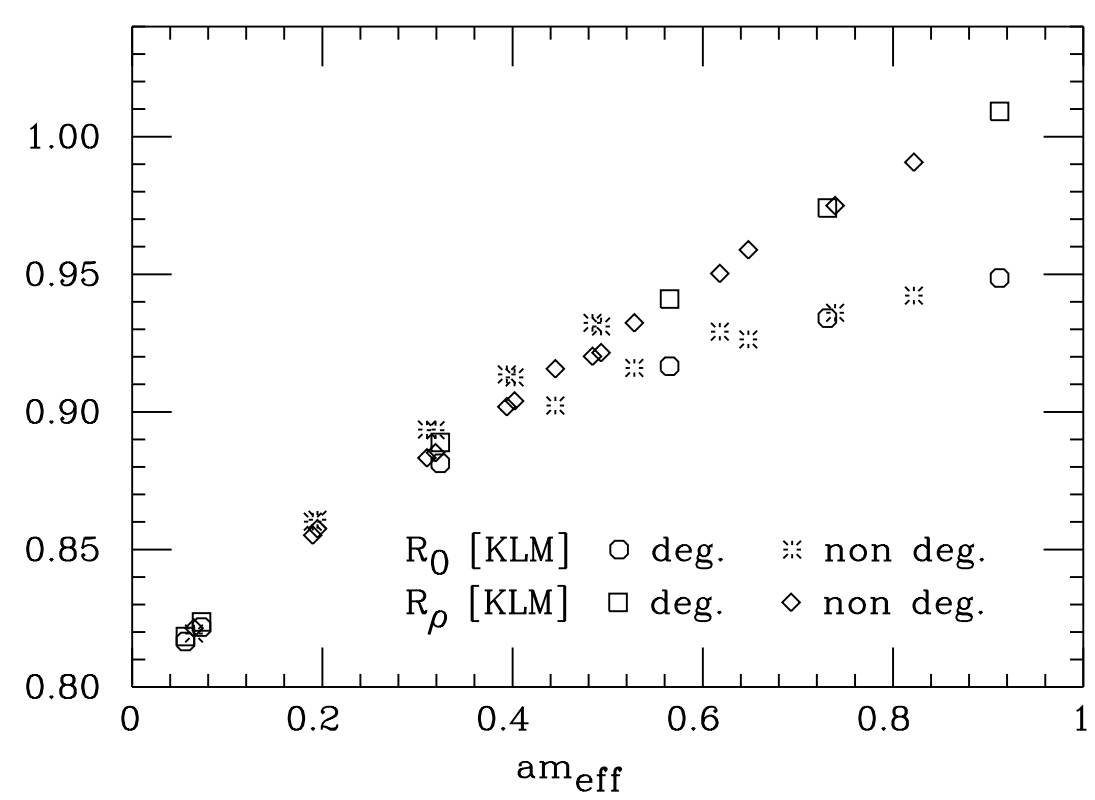

Figure 4: Same as in figure 3 with KLM corrections.

in remarkable agreement with its determination from light quark masses; cf. eq. (129). Notice that the two results have been obtained from completely independent numerical simulations.

The values of the coefficients $B$ in table 8 provide an estimate of the size of the correction coming from a term which is linear in the quark mass. Since the action is tree-level improved, this term must be of $\mathcal{O}\left(g^{2} a\right)$ or higher. We find $B \sim 0.15$, for both ratios $R_{0}$ and $R_{\rho}$. As expected, these coefficients are practically unaffected by the KLM corrections. Remarkably, however, we find that their value is almost "universal", in the sense that it is almost independent on the external states of the ME of the vector current.

On the other hand, the values of the coefficients $C$ and $D$ of the quadratic terms are not universal. For the ratio $R_{0}$, the large value of coefficient $D$ is found to be significantly reduced by the KLM correction.

We stress again that all these $\mathcal{O}(a)$ effects are quite relevant in the region which, for current values of the lattice spacing, corresponds approximately to the charm quark mass. This in turn is crucial for the phenomenological studies of the heavy flavour physics on the lattice.

In an attempt to reduce the large $\mathcal{O}\left(g^{2} a\right)$ effects observed in the data, we have also tried to modify the definition of the improved operators by varying the $z$ parameter in eq. (84). Specifically, we have considered the values $z=0$ and $z=0.5$ besides the standard choice $z=1$ analyzed so far. We find that in all cases the size of the $\mathcal{O}\left(g^{2} a\right)$ contributions is approximately the same. Thus, no specific choice of $z$ appears to improve the situation. 


\begin{tabular}{|c|c|c|c|c|c|}
\hline$K_{1}$ & $K_{2}$ & $a m_{e f f}$ & $Z_{V}$ & $Z_{V}^{K L M}$ & $Z_{V}\left(R_{0}^{K L M}\right)$ \\
\hline 0.1150 & 0.1150 & 0.912 & $0.62(1)$ & $1.01(1)$ & 0.949 \\
0.1200 & 0.1200 & 0.731 & $0.68(1)$ & $0.93(1)$ & 0.934 \\
0.1250 & 0.1250 & 0.565 & $0.74(1)$ & $0.88(1)$ & 0.917 \\
0.1330 & 0.1330 & 0.324 & $0.79(1)$ & $0.84(1)$ & 0.881 \\
0.1425 & 0.1425 & 0.073 & $0.80(2)$ & $0.80(2)$ & 0.822 \\
0.1432 & 0.1432 & 0.056 & $0.79(2)$ & $0.79(2)$ & 0.817 \\
\hline
\end{tabular}

Table 9: The $R C Z_{V}$, for the Clover action, obtained from the axial WI with and without the $K L M$ correction. For comparison, the values of the ratio $R_{0}^{K L M}$ are also shown in the last column.

\subsection{WI Determination of $Z_{V}$ and $Z_{A}$ with the Clover Ac- tion and Heavy Quark Masses}

In this subsection we present the Clover results for the RC's $Z_{V}$ and $Z_{A}$ obtained from a direct evaluation of the axial lattice chiral WI. This calculation has been performed by using the set of gauge configurations and quark propagators of subsection 7.3 .

A determination of $Z_{V}$ can be obtained by considering the axial WI of eq. (76) . In the Clover case, the right-hand side of this equation must be corrected for an $\mathcal{O}(a)$ term as shown in eq. (88), so that the resulting WI has the form:

$$
2 \rho^{12} \int d x \int d \vec{y}\left\langle P^{12}(x) A_{0}^{21}(y) V_{0}^{22}(0)\right\rangle=-\left(\frac{1}{Z_{V}^{22}}-a \rho^{12}\right) \int d \vec{y}\left\langle A_{0}^{21}(y) A_{0}^{12}(0)\right\rangle
$$

By computing $\rho$ from eq. (64) (with improved operators and symmetric derivative $\bar{\nabla}^{0}$ ) and the two correlation functions appearing in the above WI, we can solve for $Z_{V}$.

The KLM improvement to eq. (132) involves both the standard KLM factors, which correct the two- and three-point correlation functions, and the KLM correction to the ratio $\rho$, given by eq. (121). The results for $Z_{V}$ obtained from this WI are then presented in table 9, with and without the KLM corrections. For a comparison, we also show in the table the values of $Z_{V}$ corresponding to the ratio $R_{0}^{K L M}$, which have already been given in table 7 .

Let us first consider in table 9 the values of $Z_{V}$ obtained from the WI (132) without the KLM corrections. As the bare quark mass increases from $a m \simeq 0$ up to $a m \simeq 0.9$ we find that $Z_{V}$ decreases by approximately $25 \%$ due to finite cutoff effects. This is to be contrasted to the increase by about $25 \%$ of the $R_{0}$ estimate of $Z_{V}$ (sixth column of the table) in the same mass interval. The two determinations only agree at very small values of the quark mass.

When we apply the KLM prescription we see from the fifth column of table 9 a dramatic change in the estimates of $Z_{V}$. This effect is mainly due to the different normalization of the ratio $\rho$, and signals the presence of large $\mathcal{O}\left(a^{2} m^{2}\right)$ corrections. However, in spite of the KLM correction, the variation of $Z_{V}$ with the quark mass is 


\begin{tabular}{|c|c|c|c|c|}
\hline$K_{1}$ & $K_{2}$ & $a m_{\text {eff }}$ & $Z_{A}$ & $Z_{A}^{K L M}$ \\
\hline 0.1150 & 0.1150 & 0.912 & $0.87(1)$ & $1.07(1)$ \\
0.1200 & 0.1200 & 0.731 & $0.92(1)$ & $1.05(1)$ \\
0.1250 & 0.1250 & 0.565 & $0.97(1)$ & $1.05(1)$ \\
0.1150 & 0.1432 & 0.484 & $0.88(1)$ & $0.93(1)$ \\
0.1200 & 0.1432 & 0.394 & $0.91(1)$ & $0.95(1)$ \\
0.1330 & 0.1330 & 0.324 & $1.04(1)$ & $1.06(1)$ \\
0.1250 & 0.1432 & 0.310 & $0.95(1)$ & $0.97(1)$ \\
0.1330 & 0.1432 & 0.190 & $1.01(1)$ & $1.02(1)$ \\
0.1425 & 0.1425 & 0.073 & $1.11(2)$ & $1.11(2)$ \\
0.1425 & 0.1432 & 0.065 & $1.12(2)$ & $1.12(2)$ \\
0.1432 & 0.1432 & 0.056 & $1.12(2)$ & $1.12(2)$ \\
\hline
\end{tabular}

Table 10: The $R C Z_{A}$ for the Clover action, with and without KLM corrections, for different values of the quark masses.

still of about $25 \%$. Comparing the results of the last two columns of the table, we note a good agreement between the KLM corrected $Z_{V}$ estimates obtained with the WI and Ratio methods. This suggests that the KLM correction has adjusted the $\mathcal{O}\left(a^{2}\right)$ terms in such a way that the resulting values of the RC's exhibit a more universal behaviour, approximately independent of the specific correlation functions used for their computation.

Fitting $Z_{V}$ as in eq. (125), we obtain an estimate of the RC's with presumably negligible $\mathcal{O}(\mathrm{am})$ discretization effects. We quote:

$$
Z_{V}^{e f f}=0.80(2) \quad, \quad \text { from WI }
$$

in perfect agreement with the previous determinations from the Ratio method (eqs. (129) and (131)).

The RC of the local axial current $Z_{A}$, can be computed from the WI of eq. (88), by choosing suitable spatial Lorentz indices of the currents: $\nu=\rho=1,2,3$. Also in this case we find it convenient to take the flavour -2 and -3 quarks degenerate in mass, so that the WI only involves the RC's $Z_{A}^{12}$ and $Z_{V}^{22}$. For the latter, we use the values obtained from the ratio $R_{0}$ (at the corresponding quark masses), which is affected by a smaller statistical error, compared to the determination coming from the axial WI. Also for $Z_{A}$ we have considered the results obtained with and without the KLM prescription, which involves in this case corrections to the correlation functions, to the ratio $\rho$ and to $Z_{V}$ itself. The resulting values of $Z_{A}$ are presented in table 10 and are plotted in figure 5 as a function of the effective mass $a m_{e f f}=\left(a m_{1}+a m_{2}\right) / 2$.

As in the case of the vector current, we find from table 10 and figure 5 a large dependence of $Z_{A}$ on the two quark masses. For values of the effective quark mass of the order of $a m \simeq 0.5$ such a dependence can affect the value of the RC by approximately $20 \%$. When we fit $Z_{A}$ as in eq. (125), we find for the coefficient of the linear term 


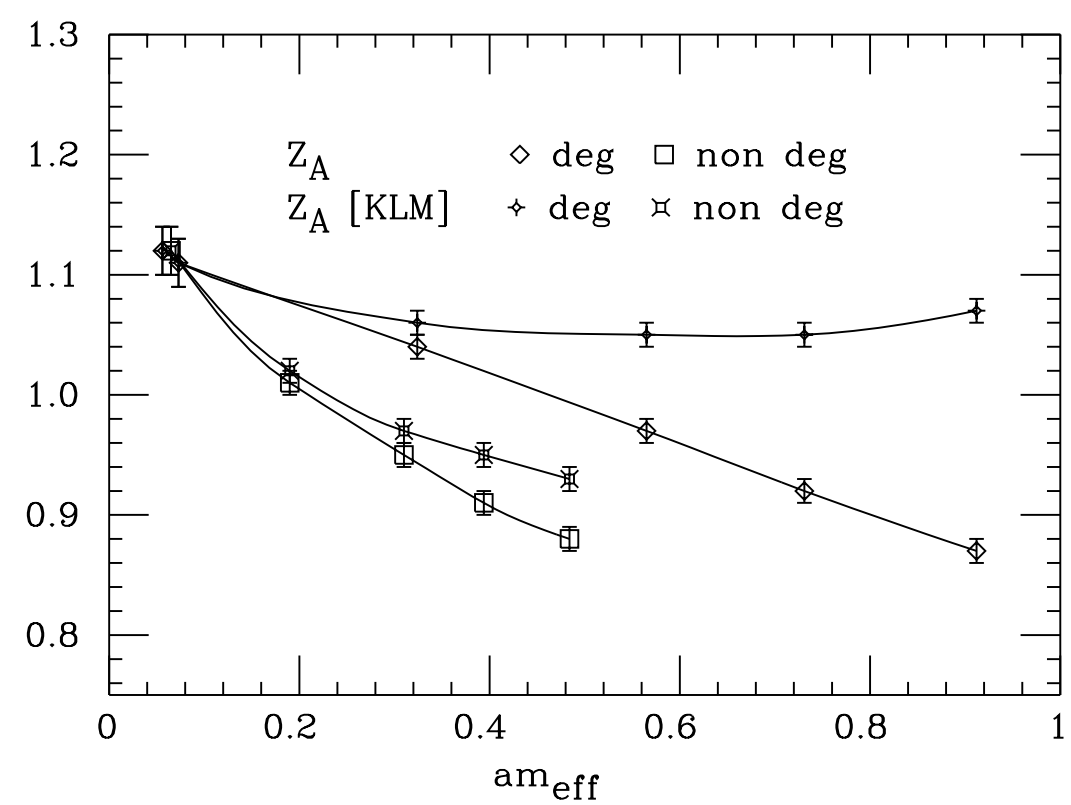

Figure 5: The $R C Z_{A}$ for the Clover action, with and without KLM corrections, as a function of the effective quark mass. Lines are only guides to the eye.

the value $B \simeq-0.13$. This value is of opposite sign but similar in magnitude to the coefficient found for $Z_{V}$. This indicates the presence of large $\mathcal{O}\left(g^{2} a m\right)$ corrections in both cases. From figure 5 we also notice that points corresponding to degenerate and non degenerate quark masses in the current do not lie on the same curve, indicating the presence of a large dependence on the difference of the two quark masses. From the fit we obtain $D \simeq-0.17$ for both uncorrected and KLM corrected data. Therefore, we find that the KLM corrections do not improve the finite cutoff effects observed in the numerical estimates of $Z_{A}$.

From the zeroth order coefficient in the fit of $Z_{A}$ we also derive an estimate of the value of the $\mathrm{RC}$ with presumably negligible $\mathcal{O}(\mathrm{am})$ effects. We quote:

$$
Z_{A}^{e f f}=1.10(2)
$$

in good agreement with the independent determination at light quark masses, presented in table 6 .

\section{Further Improvements and Future Outlook}

The results presented in this paper lead to the following conclusions:

- Applying various recipes of BPT gives RC's which are consistent to each other within $10 \%$ and which differ from those obtained from SPT by at most $20 \%$. In most (but not all) cases, perturbative improvements based on BPT can lead to more reliable estimates of the RC's, in the sense that they are in better agreement with those obtained non-perturbatively. 
- At present values of the lattice cutoff, non-perturbative estimates of the RC's based on lattice WI's, suffer, in the Wilson case, from large discretization errors of about $20 \%$. When tree-level Clover improvement is implemented, such systematic effects decrease significantly for correlation functions computed at small quark mass. They remain large, however, as the quark mass increases ( $a m \gtrsim 0.2$ ). We have checked that this situation persists for various choices of the field rotation, characteristic of tree-level $\mathcal{O}(a)$ improvement (i.e. different choices of $z$ in eq. (84)).

- A very systematic analysis of the KLM corrections has been carried out, both with Wilson and Clover actions. In the Wilson case, the KLM factors sometimes succeed and sometimes fail to correct large $\mathcal{O}(\mathrm{am})$ effects, depending on the correlation function examined. Moreover, even in the range of small quark masses, large residual $\mathcal{O}(a)$ errors remain, as can be seen from the discrepancies in the $Z_{V}$ estimates from different correlation functions. In the Clover case, KLM cannot correct the observed large $\mathcal{O}\left(g_{0}^{2} a m\right)$ systematic errors.

The implication of these results is that, with present day lattice cutoffs of about $2-4 \mathrm{GeV}(\beta=6.0-6.4)$, simulations of QCD phenomenology in the charm region (e.g. studies of $f_{D}$ or $D$-meson semileptonic decays) suffer from sizeable finite cutoff errors. This limits the predictability of lattice QCD for heavy flavour physics. In order to circumvent this problem, and given the impracticability of performing the simulations at significantly smaller lattice spacings, one must seek to improve the lattice convergence to the continuum limit. In this work, we have examined two such efforts, namely Clover tree-level improvement and KLM matching. Here we comment briefly on the recently proposed extensions of these efforts, [32] and [39].

The aim of ref. [32] is non-perturbative $\mathcal{O}(a)$ improvement. To achieve this, the Wilson action must be modified by adding the (dimension-5) Clover term of eq. (77) with the coefficient $c_{S W}$ determined non-perturbatively. In addition, a dimension- $d$ renormalized operator must be cast in the form

$$
\hat{O}=Z_{O}\left(g^{2}, a \mu\right)\left[1+b_{O}\left(g^{2}\right) a m\right]\left[O+a \sum_{n} c_{n}\left(g^{2}\right) \delta O_{n}\right]
$$

where the $\delta O_{n}$ 's are the dimension- $(d+1)$ operators which are allowed to mix with $O$. The constants $b_{O}$ and $c_{n}$ can either be determined in PT or non-perturbatively. In PT they are known at tree-level and, in some cases, at 1-loop [56]. For example, for bilinear local quark operators, $c_{n}=0$ and $b_{0}=1$ correspond to the tree-level Clover improvement discussed in this work, with field rotations determined at $z=1$. For a full $\mathcal{O}(a)$ improvement, all the improving coefficients must be computed non-perturbatively. In ref. [32] the coefficients $c_{S W}$ of the Clover term and $c_{A}$ of the axial current have been computed non-perturbatively, with the aid of lattice chiral WI's; for the coefficient $c_{V}$ of the vector current see ref. [57]. As shown in [34], chiral WI's cannot be used in the spirit of [32] for the determination of the coefficients $b_{O}$ (with the exception of $b_{V}$ of the vector current computed in [33]). Two methods for the computation of $c_{O}$ and $b_{O}$, 
which achieve $\mathcal{O}(a)$ improvement at non-zero quark masses, can be found in refs. 34] and [35]. In this way, the approach to the continuum limit is accelerated to $\mathcal{O}\left(a^{2}\right)$.

The proposal of ref. [39] can be summarized as follows. We saw that the KLM matching consists in the redefinition

$$
\hat{O}=Z_{O}\left(g_{0}^{2}, a \mu\right) F_{O}(a m) O
$$

The KLM factor $F_{O}$ has been worked out at zero spatial momentum in the free theory and for any value of the quark mass. The authors of [39] propose to extend this calculation beyond the free theory. They claim that it is possible to perform the lattice to continuum matching, again at zero spatial momentum but for any value of the quark mass, order by order in PT and to all orders in am. However, this matching has not yet been worked out beyond tree-level.

We believe that the non-perturbative Clover improvement of on-shell ME's, proposed in [32], is the most promising way of controlling discretization errors at present day values of the UV cutoff. Our analysis of the tree-level Clover improvement at large quark masses strongly suggests that it is crucial to implement the extension of this program beyond the chiral limit [34, 35]. This will hopefully render lattice computations of heavy flavour physics extremely accurate.

\section{Acknowledgements}

We wish to thank C.R. Allton, G.Martinelli and S.Petrarca for their early participation in this work. We are very grateful to the above and also to G.C Rossi, M.Talevi and M.Testa for their constant help and many suggestions. We acknowledge useful discussions with A.Kronfeld and P.Mackenzie. We thank the members of the ELC collaboration for providing us with their data, which we have used in the $\beta=6.4$ numerical computation with Wilson fermions.

\section{Appendix: The Calculation of $q^{*}$}

We give here the essential details and discuss some subtleties concerning the calculation of $q^{*}$ for the RC's $Z_{V}, Z_{A}$ and the ratio $Z_{S} / Z_{P}$. For definitiveness, we consider the $\overline{\mathrm{MS}}$ renormalization prescription in the $N D R$ scheme. The general 1-loop expression of eq. (26), for the three quantities of interest, becomes

$$
\begin{aligned}
Z_{V} & =1-\frac{g_{0}(a)^{2}}{(4 \pi)^{2}} C_{F}\left[C_{V}^{L A T}+C_{\Sigma}^{L A T}\right] \\
Z_{A} & =1-\frac{g_{0}(a)^{2}}{(4 \pi)^{2}} C_{F}\left[C_{A}^{L A T}+C_{\Sigma}^{L A T}\right] \\
\frac{Z_{S}}{Z_{P}} & =1-\frac{g_{0}(a)^{2}}{(4 \pi)^{2}} C_{F}\left[C_{S}^{L A T}-C_{P}^{L A T}\right]
\end{aligned}
$$


These expressions are easily derived from the discussion preceeding subsec.3.1. For convenience, we have rescaled the lattice constants $C^{L A T}$ by the colour factor $C_{F}=$ $4 / 3$, which now appears explicitly. Note that none of the above quantities depends on the renormalization scale $a \mu$. Consequently, the $q^{*}$ 's of these quantities are also independent of $a \mu$.

For the Wilson action, the terms on the r.h.s of the above expressions have been worked out in [2, 3]:

$$
\begin{aligned}
C_{V}^{L A T}+C_{\Sigma}^{L A T} & =1-\left[\frac{1}{4} I_{1}-\frac{1}{2} I_{2}-I_{3}+I_{\Sigma}\right] \\
C_{A}^{L A T}+C_{\Sigma}^{L A T} & =1-\left[\frac{1}{4} I_{1}+\frac{1}{2} I_{2}-I_{3}+I_{\Sigma}\right] \\
C_{S}^{L A T}-C_{P}^{L A T} & =-2 I_{2}
\end{aligned}
$$

where the finite integrals $I_{1}, I_{2}, I_{3}$ and $I_{\Sigma}$ are reproduced here for completeness:

$$
\begin{aligned}
& I_{1}=4 \pi^{2} \int_{-\pi}^{\pi} \frac{d^{4} q}{(2 \pi)^{4}}\left[\frac{\frac{4}{3}\left(\Delta_{1} \Delta_{4}-\Delta_{5}\right)+2 r^{2} \Delta_{4}^{2}+4 r^{4} \Delta_{1}^{2} \Delta_{4}}{\Delta_{1} \Delta_{2}^{2}}+\frac{4 \Delta_{4}\left(\Delta_{3}^{2}-2 \Delta_{1} \Delta_{3}\right)}{\Delta_{1}^{3} \Delta_{2}^{2}}\right] \\
& I_{2}=4 \pi^{2} r^{2} \int_{-\pi}^{\pi} \frac{d^{4} q}{(2 \pi)^{4}}\left[\frac{4 \Delta_{1}\left(4-\Delta_{1}\right)-\Delta_{4}}{\Delta_{2}^{2}}\right] \\
& I_{3}=4 \pi^{2} \int_{-\pi}^{\pi} \frac{d^{4} q}{(2 \pi)^{4}}\left[\frac{-4 \Delta_{5}}{3 \Delta_{1} \Delta_{2}^{2}}+\frac{\frac{1}{3} \Delta_{4}+2 r^{2} \Delta_{4}+4 r^{4} \Delta_{1}^{2}}{\Delta_{2}^{2}}\right] \\
& I_{\Sigma}=4 \pi^{2} \int_{-\pi}^{\pi} \frac{d^{4} q}{(2 \pi)^{4}}\left[\frac{\Delta_{4}}{4 \Delta_{1}^{3} \Delta_{2}}\left(\Delta_{3}-r^{2} \Delta_{1}^{2}\right)+\frac{\Delta_{5}}{4 \Delta_{1}^{2} \Delta_{2}}-\frac{r^{2} \Delta_{6}}{\Delta_{2}}-\frac{\Delta_{1}}{2}\right]
\end{aligned}
$$

with

$$
\begin{aligned}
\Delta_{1} & =\sum_{\mu} \sin ^{2}\left(q_{\mu} / 2\right) \quad ; & \Delta_{2} & =\sum_{\mu} \sin ^{2}\left(q_{\mu}\right)+4 r^{2}\left[\sum_{\mu} \sin ^{2}\left(q_{\mu} / 2\right)\right]^{2} \\
\Delta_{3} & =\sum_{\mu} \sin ^{4}\left(q_{\mu} / 2\right) ; & \Delta_{4} & =\sum_{\mu} \sin ^{2}\left(q_{\mu}\right) \\
\Delta_{5} & =\sum_{\mu} \sin ^{2}\left(q_{\mu}\right) \sin ^{2}\left(q_{\mu} / 2\right) & ; & \Delta_{6}=\sum_{\mu} \cos \left(q_{\mu}\right)
\end{aligned}
$$

In the present work, the Wilson parameter has been set to unity; $r=1$.

The recipe for finding $q^{*}$ is most straightforward for the ratio $Z_{S} / Z_{P}$. In order to implement eq. (31), we must simply compute the 4-dimensional integral $I_{2}$ and also the corresponding integral $L_{2}$, obtained by multiplying the integrand of $I_{2}$ by a $\ln \left(q^{2}\right)$. $L_{2}$ is also finite, and therefore calculable. Thus, for $Z_{S} / Z_{P}$ we have

$$
\ln \left(q^{* 2}\right)=\frac{L_{2}}{I_{2}}
$$


We note in passing that the recipe of eq. (31) is not univocal, even for this simple case: the internal momentum $q$ of the integral $I_{2}$, being a dummy variable, can be chosen with a certain degree of arbitrariness (in particular, translations of $q$ ) which does not affect the final answer, since the integrand is made up of translationally invariant propagators. However, such changes in $q$ will affect the logarithm in the integrand of $L_{2}$, and thus will give us a different $q^{*}$. This is only an academic problem, because $q^{*}$ is only meant to be a typical scale of the process under examination. Thus, estimates of $q^{*}$ which differ by the choice of internal momenta $q$ are all equally acceptable.

The calculation of $q^{*}$ for $Z_{V}$ and $Z_{A}$ is more complicated. Besides $L_{2}$, we also need $L_{1}$ and $L_{3}$ (again obtained from $I_{1}$ and $I_{3}$, by modifying their integrands by $\ln \left(q^{2}\right)$ ). $L_{1}$ and $L_{3}$ are also finite, so this causes no serious problem. The real complication arises from the unity on the r.h.s of eq. (138). This constant is obtained as a difference of two integrals which diverge logarithmically with the external momentum $a p^{2}$. We write them out explicitly. The first one arises from the divergent part of the projected amputated Green functions $\Gamma_{V}(p)$ and $\Gamma_{A}(p)$ :

$$
\begin{aligned}
\hat{I}_{\Gamma}(a p) & =16 \pi^{2} \int_{-\pi}^{\pi} \frac{d^{4} q}{(2 \pi)^{4}} \frac{\sum_{\rho} \sin ^{2} q_{\rho}}{\left[4 \sum_{\mu} \sin ^{2}\left(\frac{q_{\mu}}{2}\right)\right]^{2}\left[4 \sum_{\nu} \sin ^{2}\left(\frac{q_{\nu}+a p_{\nu}}{2}\right)\right]} \\
& =-\ln \left(a^{2} p^{2}\right)-\gamma_{E}+F_{0001}+5 / 2+\mathcal{O}(a p)
\end{aligned}
$$

whereas the second integral arises from the divergent part of the self-energy:

$$
\begin{aligned}
\hat{I}_{\Sigma}(a p) & =-16 \pi^{2} \int_{-\pi}^{\pi} \frac{d^{4} q}{(2 \pi)^{4}} \frac{\sum_{\rho} \sin \left(q_{\rho}+a p_{\rho}\right) \sin \left(q_{\rho}\right)}{\left[4 \sum_{\mu} \sin ^{2}\left(\frac{q_{\mu}}{2}\right)\right]^{2}\left[4 \sum_{\nu} \sin ^{2}\left(\frac{q_{\nu}+a p_{\nu}}{2}\right)\right]} \\
& =\ln \left(a^{2} p^{2}\right)+\gamma_{E}-F_{0001}-3 / 2+\mathcal{O}(a p)
\end{aligned}
$$

The integration (in the limit $a p \rightarrow 0$ ) has been performed with the aid of the tabulated integrals of [58]. Thus, the factor +1 on the r.h.s. of the first two eqs. (138) is obtained from the integral $\hat{I}_{S U M}=\hat{I}_{\Gamma}+\hat{I}_{\Sigma}$ :

$$
\begin{aligned}
\hat{I}_{S U M} & =16 \pi^{2} \int_{-\pi}^{\pi} \frac{d^{4} q}{(2 \pi)^{4}} \frac{\sum_{\rho}\left[\sin \left(q_{\rho}\right)-\sin \left(q_{\rho}+a p_{\rho}\right)\right] \sin \left(q_{\rho}\right)}{\left[4 \sum_{\mu} \sin ^{2}\left(\frac{q_{\mu}}{2}\right)\right]^{2}\left[4 \sum_{\nu} \sin ^{2}\left(\frac{q_{\nu}+a p_{\nu}}{2}\right)\right]} \\
& =1+\mathcal{O}(a p)
\end{aligned}
$$

The above integral is finite. Its contribution to the RC's is given by its limit $a p \rightarrow 0$. We note that, in this limit the integrand of $\hat{I}_{S U M}$ vanishes, except for $q_{\mu}=0$. Thus, the integral is dominated by the region of small $q$. This enables us to substitute the integrand by its small $q$ and $a p$ limit and take it over the whole $q$ range:

$$
\hat{I}_{S U M}=-\frac{1}{\pi^{2}} \int_{-\infty}^{+\infty} d^{4} q \frac{a p \cdot q}{q^{4}(q+a p)^{2}}
$$


We introduce the standard Feynman parameters, and after some trivial algebraic manipulations obtain:

$$
\hat{I}_{S U M}=-\frac{2}{\pi^{2}} \int_{0}^{1} d x(1-x) \int_{-\infty}^{+\infty} d^{4} q \frac{a p \cdot q}{\left[(q+x a p)^{2}+(a p)^{2} x(1-x)\right]^{3}}
$$

We next shift the integration variable $q \rightarrow q+x a p$ and pass over to polar coordinates in $q$-space. Integrating the polar angles leaves us with

$$
\hat{I}_{S U M}=2(a p)^{2} \int_{0}^{1} d x(1-x) \int_{0}^{+\infty} d q^{2} \frac{q^{2}}{\left[q^{2}+(a p)^{2} x(1-x)\right]^{3}}
$$

Finally, we perform a further change of variables $q^{2} \rightarrow q^{2} /\left[a^{2} p^{2} x(1-x)\right]$, and carry out the integration in $x$ to obtain

$$
\hat{I}_{S U M}=2 \int_{0}^{+\infty} d q^{2} \frac{q^{2}}{\left(q^{2}+1\right)^{3}}
$$

The above is easily seen to be equal to 1 , which is the final result of eq. (144).

Now the application of eq. (31) requires the introduction of $\ln \left(q^{2}\right)$ in the integrand of $\hat{I}_{S U M}$. However, it is not obvious at which stage of the successive integrations from eq. (144) to eq. (148) this should be done. For example, if we were to introduce $\ln \left(q^{2}\right)$ in eq. (147), we would find a result proportional to $\ln \left(a^{2} p^{2}\right)$. This does not make sense, since it yields a $q^{*}$ which depends on the momenta of the external states and, even worse, this ap dependence is singular. Thus we prefer to introduce the $\ln \left(q^{2}\right)$ in the integrand of eq. (148):

$$
L_{S U M}=2 \int_{0}^{+\infty} d q^{2} \frac{q^{2} \ln \left(q^{2}\right)}{\left(q^{2}+1\right)^{3}}
$$

The above integration can be easily performed; we find $L_{S U M}=1$, which is a $p$ independent constant. Thus, $q^{*}$ for $Z_{V}$ is given by

$$
\ln \left(q^{* 2}\right)=\frac{1-\left[\frac{1}{4} L_{1}-\frac{1}{2} L_{2}-L_{3}+L_{\Sigma}\right]}{1-\left[\frac{1}{4} I_{1}-\frac{1}{2} I_{2}-I_{3}+I_{\Sigma}\right]}
$$

For $Z_{A}$ we have

$$
\ln \left(q^{* 2}\right)=\frac{1-\left[\frac{1}{4} L_{1}+\frac{1}{2} L_{2}-L_{3}+L_{\Sigma}\right]}{1-\left[\frac{1}{4} I_{1}+\frac{1}{2} I_{2}-I_{3}+I_{\Sigma}\right]}
$$

The Clover case presents no extra conceptual difficulties. Everything is like in the Wilson case, except that on the r.h.s. of eqs. (138) we have more finite integrals, which arise from the extra (Clover) term in the action and the $\not D$ field rotations of subsection 5.2. These finite integrals are listed in refs. [0, 8]. 
A final comment is in place here. A shortcut to all the subtleties related to $L_{S U M}$ would be to simply ignore the contribution of this integral to $q^{*}$. In this case, $q^{*}$ is

obtained from the last two equations, with the +1 's omitted from both numerator and denominator. With this $q^{*}$ the most affected RC's are $Z_{V}$ and $Z_{A}$ of the Clover action. Their values change by less than $2 \%$, for all boosting recipes of the coupling.

\section{References}

[1] B. Meyer and C. Smith, Phys. Lett. B123 (1983) 62.

[2] G. Martinelli and Y.C. Zhang, Phys.Lett. B123(1983) 433; Phys. Lett. B125 (1983) 77.

[3] A. Gonzalez Arroyo, F.J. Yndurain and G. Martinelli, Phys. Lett. B117 (1982) 437.

[4] R. Groot, J. Hoek and J. Smit Nucl Phys. B237 (1984) 111.

[5] G. Martinelli, Phys. Lett. B141 (1984) 395.

[6] C. Bernard, A. Soni and T. Draper, Phys. Rev. D36 (1987) 3224.

[7] E. Gabrielli et al., Nucl.Phys. B362 (1991) 475.

[8] A. Borrelli, R. Frezzotti, E. Gabrielli and C. Pittori, Nucl. Phys. B409 (1993) 382 .

[9] R. Frezzotti, E. Gabrielli, C. Pittori and G.C. Rossi, Nucl. Phys. B373 (1991) 781.

[10] C.J. Morningstar, Nucl. Phys. B(Proc. Suppl.)47 (1996) 92.

[11] L. Maiani and G. Martinelli, Phys.Lett. B178 (1986) 265.

[12] G. Martinelli and C.T. Sachrajda, Nucl. Phys. B306 (1988) 865; Nucl. Phys. B316 (1989) 305.

[13] C.T. Sachrajda, Nucl. Phys. B(Proc.Suppl) 9 (1989) 121.

[14] M. Crisafulli, G. Martinelli, V.J. Hill and C.T. Sachrajda, Phys. Lett. B223 (1989) 90.

[15] G.Martinelli, C.T.Sachrajda and A.Vladikas, Nucl. Phys. B358 (1991) 212.

[16] G. Martinelli, C.T. Sachrajda, G. Salina and A. Vladikas, Nucl. Phys. B378 (1992) 591; Nucl. Phys. B397 (1993) 479.

[17] G. Martinelli, S. Petrarca, C.T. Sachrajda and A. Vladikas, Phys. Lett. B311 (1993) 241; Phys. Lett. B317 (1993) 660.

[18] M. Crisafulli, V. Lubicz, G. Martinelli and A. Vladikas, Nucl. Phys. B(Proc. Suppl.)42 (1995) 400. 
[19] M. Paciello, S. Petrarca, B. Taglienti and A. Vladikas Phys. Lett. B341 (1994) 187.

[20] D.S. Henty, R.D. Kenway, B.J. Pendleton and J.I. Skullerud, Phys. Rev D51 (1995) 5323.

[21] JLQCD Collaboration, S. Aoki et al., Nucl.Phys.B(Proc.Suppl.) 53 (1997) 349; see also preprint hep-lat/9705035.

[22] G.Martinelli et al, Nucl.Phys.B445(1995)81.

[23] A. Donini et al, Phys. Lett. B360 (1996) 83.

[24] M. Göckeler et al., Nucl. Phys. B(Proc.Suppl) 47 (1996) 493.

[25] A. Vladikas, Nucl. Phys. B(Proc. Suppl.)47 (1996) 84.

[26] A. Donini, V. Giménez, G. Martinelli, G.C. Rossi, M. Talevi, M. Testa and A. Vladikas, Nucl.Phys.B(Proc.Suppl.) 53 (1997) 883.

[27] G.C. Rossi, Nucl.Phys.B(Proc.Suppl.) 53 (1997) 3.

[28] G. Martinelli, Nucl. Phys. B(Proc. Suppl.)42 (1995) 127.

[29] G.P. Lepage and P.B. Mackenzie, Phys. Rev. D48 (1993) 2250.

[30] B. Sheikholeslami and R. Wohlert, Nucl. Phys. B259 (1985) 572.

[31] G.Heatlie et al, Nucl. Phys. B352 (1991) 266.

[32] K. Jansen et al.,Phys. Lett. B372 (1996) 275;

M. Lüscher, S. Sint, R. Sommer and P. Weisz, Nucl. Phys. B 478 (1996) 365;

M. Lüscher et al., Nucl. Phys. B491 (1997) 323.

[33] M. Lüscher, S. Sint, R. Sommer and H. Wittig, Nucl. Phys. B 491 (1997) 344.

[34] G. Martinelli, G.C. Rossi, C.T. Sachrajda, S. Sharpe, M. Talevi and M. Testa, Phys. Lett. B411 (1997) 141.

[35] C. Dawson, V. Lubicz, G. Martinelli, G.C. Rossi, C.T. Sachrajda, S. Sharpe, M. Talevi and M. Testa, in preparation.

[36] G.P. Lepage, Nucl.Phys. B(Proc.Suppl.)26 (1992) 45.

[37] A.S. Kronfeld, Nucl.Phys. B(Proc.Suppl.)30 (1993) 445.

[38] P. B. Mackenzie, Nucl.Phys. B(Proc.Suppl.)30 (1993) 35.

[39] A.X. El-Khadra, A.S. Kronfeld and P.B. Mackenzie, Phys. Rev. D55 (1997) 3933

[40] K.G.Wilson, Phys. Rev. D10 (1974) 2445.

[41] K.G.Wilson, in "New Phenomena in Subnuclear Physics" (Erice 1975), ed. A.Zichichi (New York, Plenum, 1975).

[42] C.R.Allton et al, Nucl.Phys.B431(1994)667.

[43] M. Ciuchini et al, Z.Phys. C68 (1995) 239. 
[44] S. J. Brodski, G. P. Lepage and P. B. Mackenzie, Phys. Rev. D28 (1983) 228.

[45] G. Parisi, in "High Energy Physics - 1980", Proceedings of the XXth International Conference, Madison, Wisconsin, eds. L. Durand and L. G. Pondrom (American Institute of Physics, New York, 1981).

[46] C.R. Allton et al., Phys. Lett. B326 (1994) 295.

[47] M. Crisafulli et al., Phys. Lett. B369 (1996) 325.

[48] L.H. Karsten and J. Smit, Nucl.Phys. B183 (1981) 103.

[49] M. Bochicchio et al., Nucl.Phys. B262 (1985) 331.

[50] G. Curci, Phys. Lett. B167 (1986) 265.

[51] H.W. Hamber and C.M. Wu, Phys. Lett. B133 (1983)351; Phys. Lett. B136 (1984)255;

W. Wetzel, Phys. Lett. B136 (1984) 407;

T. Eguchi and N. Kawamoto, Nucl. Phys. B237 (1984) 572.

[52] K. Symanzik, in Mathematical Problems in Theoretical Physics, Springer Lecture Notes vol.153 (1982) 47; eds. R. Schrader, R. Seiler and D.A. Uhlenbrock;

K. Symanzik, Nucl. Phys. B226 (1983) 187;

K. Symanzik, Nucl. Phys. B226 (1983) 205.

[53] S. Naik, Phys. Lett. B311 (1993) 230.

[54] C.W. Bernard, Nucl.Phys. B(Proc.Suppl.)34 (1994) 47; Phys.Lett. B125(1983) 77 .

[55] A. Abada et.al. Nucl.Phys. B376(1992)172;

A. Abada et.al. Nucl.Phys. B416(1994)675.

[56] M. Lüscher and P. Weisz, Nucl. Phys. B479 (1996) 429;

S. Sint and P. Weisz, Nucl.Phys. B502 (1997) 251.

[57] M. Guagnelli and R. Sommer, hep-lat/9709088.

[58] R.K. Ellis and G. Martinelli, Nucl. Phys. B235 (1984) 93. 